\title{
Relativistic Calculations of Transition Amplitudes in the Helium Isoelectronic Sequence
}

\author{
W.R. Johnson, D.R. Plante, and J. Sapirstein \\ Department of Physics, University of Notre Dame, Notre Dame, IN 46556
}

June 27, 2011

\begin{abstract}
Exact relativistic wave functions for $n=1$ and $n=2$ states of heliumlike ions are used to calculate single-photon transition amplitudes for $2{ }^{1} P_{1} \rightarrow$ ${ }^{1} S_{0}, 2{ }^{3} P_{0,1,2} \rightarrow 2{ }^{3} S_{1}, 2{ }^{3} S_{1} \rightarrow 1{ }^{1} S_{0}, 2{ }^{1} P_{1} \rightarrow 2{ }^{3} S_{1}$ and $2{ }^{3} P_{1,2} \rightarrow 1{ }^{1} S_{0}$. Particular attention is given to the role of negative-energy states in bringing length and velocity forms into agreement, and related quantum electrodynamics issues. This is a reprinted version of an article with the same title published in Advances in Atomic, Molecular and Optical Physics 35, 225-329 (1995). Figures included in the original article are omitted here.
\end{abstract}

\section{Introduction}

The helium atom and heliumlike ions occupy special positions in atomic physics as the simplest multi-electron systems. Unlike atoms and ions with many electrons, the fact that only two electrons are present allows calculations of extremely high accuracy to be carried out in the nonrelativistic case. At low $Z$, the leading relativistic and QED corrections can be calculated as perturbations. However, at higher $Z$ it is desirable to start from a completely relativistic formalism. A well-known problem of any such formalism is the correct treatment of negative-energy states, which, if included improperly, lead to the continuum dissolution problem discussed by Sucher [1]. This problem can be avoided by using the no-pair Hamiltonian [1, 2], which excludes negative-energy states. In reference [3], configuration interaction (CI) techniques were used to carry out accurate calculations of energies of $n=1$ and $n=2$ states along the helium isoelectronic sequence starting from the no-pair Hamiltonian. Similar calculations were carried out in [4] using iterative techniques. It is the purpose of this paper to extend these calculations to evaluate transition amplitudes along the helium isoelectronic sequence. Specifically, we present highly accurate theoretical calculations of single-photon transition amplitudes and transition rates for $2-2$ and $2-1$ transitions in heliumlike ions with charges in the range $Z=2-100$. Amplitudes for the electric-dipole (E1) transitions $2{ }^{1} P_{1} \rightarrow 1{ }^{1} S_{0}, 2{ }^{3} P_{0,1,2} \rightarrow 2{ }^{3} S_{1}$, 
$2{ }^{1} P_{1} \rightarrow 2{ }^{3} S_{1}$ and $2{ }^{3} P_{1} \rightarrow 1{ }^{1} S_{0}$ are calculated in length-form and in velocityform. Amplitudes for the magnetic-dipole (M1) transitions $2{ }^{3} S_{1} \rightarrow{ }^{1}{ }^{1} S_{0}$ and the magnetic-quadrupole (M2) transitions $2{ }^{3} P_{2} \rightarrow 1{ }^{1} S_{0}$ are evaluated in velocity form.

Although the amplitudes are calculated using wave functions determined from previous work, there are several subtle points about the calculation that require particular care. Firstly, the amplitudes, although calculated using exact eigenfunctions of the no-pair Hamiltonian, depend on the gauge of the electromagnetic field, as first noted in Ref. [5]. This gauge dependence is reflected in slight differences between length-form and velocity-form transition amplitudes. The gauge dependence is a direct consequence of the fact that negative-energy contributions are omitted in the no-pair Hamiltonian. We will derive a commutator identity expressing the difference between length- and velocity-form amplitudes in terms of the commutator of the many-body potential with the dipole operator. This commutator is non-vanishing in the no-pair theory because positive-energy projection operators surround the many-body potential. The numerically calculated amplitudes are found to satisfy the commutator identity precisely. In Sec. 2, we describe the calculation of the transition amplitudes and give examples emphasizing the length-velocity differences.

Since transition amplitudes determined using wave functions from the nopair Hamiltonian are inherently gauge dependent, it becomes important to evaluate the missing negative-energy contributions and to make appropriate corrections to the amplitudes. For this purpose, we turn to the perturbation expansion of the $S$-matrix in QED. The $S$-matrix is gauge invariant order-by-order. The gauge invariance of the first-order $S$-matrix $S^{(1)}$ follows from an elementary identity involving the one-electron Dirac Hamiltonian. There are, of course, no negative-energy contributions to $S^{(1)}$. Negative-energy contributions first appear in electron propagators in the third-order $S$-matrix $S^{(3)}$. A second subtle point is that one must add derivative terms [6] to the usual third-order diagrams to establish the gauge-invariance of $S^{(3)}$. The origin of these derivative terms is illustrated in Sec. 3 by a third-order QED calculation of transitions in hydrogenlike ions carried out using the Gell-Mann-Low formalism [7]. These terms play a dual role in the calculation. In addition to establishing gauge invariance, they also act to effectively change the phase space available for the decay. A standard problem faced when calculating decay rates with approximate energies and wave functions is whether to use the theoretical or experimental photon energy. We will show that the derivative terms act to modify the lowest-order theoretical energies to include higher-order perturbation theory corrections, so that a fundamental justification for the use of corrected theoretical energies (which agree quite precisely with experimental energies) is provided.

The structure of $S^{(3)}$ inferred from the example in Sec. 3 is used in Sec. 4 to determine the first two terms in a gauge-invariant perturbation expansion of the transition amplitudes for heliumlike systems. We set up the perturbation theory calculation in Sec. 4 and give specific examples, concentrating particularly on the sensitive $2{ }^{3} P_{0,2} \rightarrow 2{ }^{3} S_{1}$ transitions. In these examples, contributions to the amplitudes from the positive-energy and negative-energy 
components of the propagator are determined separately. The positive- and negative-energy components of the amplitudes depend on gauge, but the sum of the positive- and negative-energy components is gauge independent. We find that for $2{ }^{3} P_{0} \rightarrow 2{ }^{3} S_{1}$ transitions, contributions from negative-energy terms to the velocity-form amplitude range up to $10 \%$ of the total amplitude for highly-charged ions. Negative-energy contributions to the length-form amplitudes remain small $\left(10^{-7}\right.$ a.u. $-10^{-8}$ a.u. $)$ for all of the electric-dipole transitions studied, in agreement with the conclusions reached in Ref. [5]. The lengthform amplitudes from the no-pair wave functions agree to within a few percent with the positive-energy components of the amplitudes from perturbation theory, the difference being primarily from omitted higher-order corrections in the perturbation expansion. Moreover, adding the negative-frequency components determined from perturbation theory to the no-pair amplitudes brings the velocity-form no-pair amplitudes into close agreement with the length-form amplitudes. Since there are negligible contributions to the length-form amplitudes from negative-energy states, we adopt the length-form no-pair amplitudes in our final tabulations for E1 transitions.

For magnetic-multipole transitions, the no-pair amplitudes again agree at the $1 \%$ level of accuracy with the positive-energy contributions from perturbation theory. We account for the missing negative-energy contributions by adding the negative-energy contributions from perturbation theory to the nopair amplitudes. Corrections to the wave functions from the Breit interaction significantly modify the negative-energy contributions, as found in an earlier calculation of the M1 decay of heliumlike argon [8]. We note that these relatively large negative-energy state effects were correctly included in earlier work on M1 decays based on perturbation theory [9], and the RRPA [10]. However, because differential equation techniques were used in those works, which automatically include both positive- and negative-energy states, this feature of the calculation was not noted at that time.

In Sec. 5, we present a summary of results, giving tables of amplitudes, line strengths and rates for the $2-2$ and $2-1$ transitions. Where possible, our calculations are compared with previous calculations and with measurements. We believe that the present values for transition amplitudes and rates are as accurate as, or more accurate than, all previous values.

\section{No-Pair Transition Amplitudes}

\section{$2.1 \quad$ No-pair Hamiltonian}

For a many-electron system, the no-pair Hamiltonian $[1,2]$ may be written $H=H_{0}+V_{C}+V_{B}$, where $H_{0}$ is the unperturbed Hamiltonian given in secondquantized form by

$$
H_{0}=\sum_{i} \epsilon_{i} a_{i}^{\dagger} a_{i} .
$$


The quantity $\epsilon_{i}$ is an eigenvalue of the one-electron Dirac equation

$$
h \phi_{i}=\epsilon_{i} \phi_{i}
$$

with

$$
h=c \boldsymbol{\alpha} \cdot \boldsymbol{p}+(\beta-1) c^{2}+V_{\mathrm{nuc}}(r)+U(r) .
$$

In Eq.(3), $V_{\text {nuc }}(r)$ is the Coulomb potential of the nucleus incorporating nuclear finite-size corrections, and $U(r)$ is a local model potential that approximates the electron-electron interaction. We make two choices of $U(r)$ here, namely,

$$
U(r)= \begin{cases}0, & \text { Coulomb-field case, } \\ v_{0}(1 s, r), & \text { Hartree case }\end{cases}
$$

where $v_{0}(1 s, r)$ is the self-consistent potential of the ground state of the heliumlike ion. The potential $U(r)$ is fixed by a ground-state HF calculation and treated as a local potential when solving the one-electron Dirac equation (2). The index $i$ in Eq.(2) represents the set of one-electron quantum numbers: the principal quantum number $n_{i}$, the angular momentum quantum number $\kappa_{i}$ $\left[\kappa_{i}=\mp\left(j_{i}+1 / 2\right)\right.$ for $\left.j_{i}=\left(l_{i} \pm 1 / 2\right)\right]$, and the magnetic quantum number $m_{i}$. The Coulomb interaction $V_{C}$ is given by

$$
V_{C}=\frac{1}{2} \sum_{i j k l} g_{i j k l} a_{i}^{\dagger} a_{j}^{\dagger} a_{l} a_{k}-\sum_{i j} U_{i j} a_{i}^{\dagger} a_{j},
$$

where the sum is over positive-energy states only. This restriction implements the positive-energy projection operators in Refs. $[1,2]$. The quantities $g_{i j k l}$ in Eq.(5) are two-electron Coulomb matrix elements:

$$
g_{i j k l}=\left\langle i j\left|\frac{1}{r_{12}}\right| k l\right\rangle,
$$

and

$$
U_{i j}=\langle i|U(r)| j\rangle .
$$

The Breit interaction $V_{B}$ is given by

$$
V_{B}=\frac{1}{2} \sum_{i j k l} b_{i j k l} a_{i}^{\dagger} a_{j}^{\dagger} a_{l} a_{k},
$$

where again the summation is restricted to positive-energy states. Here $b_{i j k l}$ are two-electron matrix elements of the instantaneous Breit operator,

$$
b_{i j k l}=-\left\langle i j\left|\frac{\boldsymbol{\alpha}_{\mathbf{1}} \cdot \boldsymbol{\alpha}_{\mathbf{2}}+\boldsymbol{\alpha}_{\mathbf{1}} \cdot \hat{\mathbf{r}}_{12} \boldsymbol{\alpha}_{\mathbf{2}} \cdot \hat{\mathbf{r}}_{12}}{2 r_{12}}\right| k l\right\rangle .
$$

Later, in our discussion of perturbation theory, we use the notation

$$
\begin{aligned}
& v_{i j k l}=g_{i j k l}+b_{i j k l}, \\
& \tilde{v}_{i j k l}=v_{i j k l}-v_{i j l k} .
\end{aligned}
$$


The no-pair Hamiltonian includes all corrections of order $\alpha^{2} Z^{2}$ a.u., but does not include QED corrections of order $\alpha^{3} Z^{4}$ a.u., such as the Lamb shift. Such corrections must be calculated separately as discussed, for example, in Ref. [4].

A two-electron state vector describing an atomic state with angular momentum $J, M$ may be written

$$
\Psi_{J M}=\sum_{i \leq j} c_{i j} \Phi_{i j}
$$

where the quantities $c_{i j}$ are expansion coefficients and where the configuration state vectors $\Phi_{i j}$ are defined by

$$
\Phi_{i j}=\eta_{i j} \sum_{m_{i} m_{j}}\left\langle j_{i} m_{i}, j_{j} m_{j} \mid J M\right\rangle a_{i}^{\dagger} a_{j}^{\dagger}|0\rangle,
$$

with

$$
\eta_{i j}=\left\{\begin{array}{cc}
1, & i \neq j \\
\frac{1}{\sqrt{2}}, & \mathrm{i}=\mathrm{j}
\end{array}\right.
$$

The quantities $c_{i j}, \Phi_{i j}$, and $\eta_{i j}$ are independent of magnetic quantum numbers. Therefore, a particular state $i$ is uniquely determined by $n_{i}$ and $\kappa_{i}$. To construct a state of even or odd parity, one must require the sum $l_{i}+l_{j}$ to be either even or odd, respectively. From the symmetry properties of the Clebsch-Gordan coefficients, it can be shown that

$$
\Phi_{i j}=(-1)^{j_{i}+j_{j}+J+1} \Phi_{j i}
$$

This relation, in turn, implies that $\Phi_{i i}$ vanishes unless $J$ is even. The wavefunction normalization condition has the form

$$
\left\langle\Psi_{J M} \mid \Psi_{J M}\right\rangle=\sum_{i \leq j} c_{i j}^{2}=1 .
$$

Substituting $\Psi_{J M}$ into the Schrödinger equation $\left(H_{0}+V\right) \Psi_{J M}=E \Psi_{J M}$, one obtains the following set of linear equations for the expansion coefficients $c_{i j}$ :

$$
\left(\epsilon_{i}+\epsilon_{j}\right) c_{i j}+\sum_{k \leq l} \eta_{i j} V(i j ; k l) \eta_{k l} c_{k l}=E c_{i j}
$$

The potential matrix in Eq.(17) is

$$
\begin{aligned}
& V(i j ; k l)=\sum_{L}(-1)^{j_{j}+j_{k}+L+J}\left\{\begin{array}{ccc}
j_{i} & j_{j} & J \\
j_{l} & j_{k} & L
\end{array}\right\} X_{L}(i j k l) \\
& \quad+\sum_{L}(-1)^{j_{j}+j_{k}+L}\left\{\begin{array}{ccc}
j_{i} & j_{j} & J \\
j_{k} & j_{l} & L
\end{array}\right\} X_{L}(i j l k) \\
& -\delta_{\kappa_{i} \kappa_{k}} \delta_{j l} U_{i k}-\delta_{\kappa_{j} \kappa_{l}} \delta_{i k} U_{j l}+(-1)^{j_{l}+j_{k}+J}\left[\delta_{\kappa_{j} \kappa_{k}} \delta_{i l} U_{j k}+\delta_{\kappa_{i} \kappa_{l}} \delta_{j k} U_{i l}\right],
\end{aligned}
$$

where the quantities $X_{L}(i j k l)$ are given by

$$
X_{L}(i j k l)=(-1)^{L}\left\langle\kappa_{i}\left\|C_{L}\right\| \kappa_{k}\right\rangle\left\langle\kappa_{j}\left\|C_{L}\right\| \kappa_{l}\right\rangle R_{L}(i j k l) .
$$


The quantities $\left\langle\kappa_{i}\left\|C_{L}\right\| \kappa_{j}\right\rangle$ are reduced matrix elements of normalized spherical harmonics, and the quantities $R_{L}(i j k l)$ are relativistic Slater integrals defined in Refs. $[3,4]$. For the case where both the Coulomb and Breit interactions are included in the Hamiltonian,

$$
X_{L}(i j k l) \rightarrow X_{L}(i j k l)+M_{L}(i j k l)+N_{L}(i j k l)+O_{L}(i j k l),
$$

where $M_{L}(i j k l), N_{L}(i j k l)$, and $O_{L}(i j k l)$ are the magnetic integrals defined in Ref. [11].

We can solve the eigenvalue problem in Eq.(17) to high accuracy using iterative techniques, as described in Ref. [4], or as a large-scale CI problem, using the methods given in Ref. [3].

\subsection{Electromagnetic interaction Hamiltonian}

The interaction Hamiltonian for a single electron with an electromagnetic field described by the vector potential $\mathbf{A}(\mathbf{r}, t)$ and the scalar potential $\phi(\mathbf{r}, t)$ is

$$
h_{I}(\mathbf{r}, t)=e\{-c \boldsymbol{\alpha} \cdot \boldsymbol{A}(\mathbf{r}, t)+\phi(\mathbf{r}, t)\},
$$

where $e=-|e|$ is the electron charge. If we assume that $h_{I}$ has time dependence $e^{-i \omega t}$ associated with an incoming photon field, we can make the replacement

$$
h_{I}(\mathbf{r}, t) \rightarrow h_{I}(\mathbf{r}, \omega) e^{-i \omega t},
$$

where

$$
h_{I}(\mathbf{r}, \omega)=e\{-c \boldsymbol{\alpha} \cdot \boldsymbol{A}(\mathbf{r}, \omega)+\phi(\mathbf{r}, \omega)\} .
$$

We will be considering gauge invariance of transition amplitudes in this paper. We wish to distinguish this kind of gauge invariance from the usual statement of the gauge invariance in QED. In that way of defining gauge invariance, the field theoretic Lagrangian remains invariant under the simultaneous transformation $\psi(x) \rightarrow e^{i \chi(x)} \psi(x), A_{\mu}(x) \rightarrow A_{\mu}(x)-(1 / e) \partial_{\mu} \chi(x)$, where the change in $A_{\mu}$ in the interaction part of the Lagrangian is compensated by the $i \partial_{\mu}$ part of the free part of the Lagrangian acting on the $e^{i \chi(x)}$ factor. Here, however, when we refer to gauge invariance, we mean that matrix elements of the interaction Hamiltonian remain unchanged when the above gauge transformation is made. The phase change of the electron fields in this case has no effect, but we will nevertheless show that the change of the Hamiltonian has a vanishing matrix element when taken between wave functions. We illustrate the present notion of gauge invariance for one-electron matrix elements in the following paragraph.

A gauge transformation

$$
\begin{aligned}
\mathbf{A}(\mathbf{r}, \omega) & \rightarrow \mathbf{A}^{\prime}(\mathbf{r}, \omega)=\mathbf{A}(\mathbf{r}, \omega)+\nabla \chi(\mathbf{r}, \omega), \\
\phi(\mathbf{r}, \omega) & \rightarrow \phi^{\prime}(\mathbf{r}, \omega)=\phi(\mathbf{r}, \omega)+i \omega \chi(\mathbf{r}, \omega),
\end{aligned}
$$

induces a change in the interaction Hamiltonian,

$$
h_{I}(\mathbf{r}, \omega) \rightarrow h_{I}^{\prime}(\mathbf{r}, \omega)=h_{I}(\mathbf{r}, \omega)+\Delta h_{I}(\mathbf{r}, \omega),
$$


where

$$
\Delta h_{I}(\mathbf{r}, \omega)=e\{-c \boldsymbol{\alpha} \cdot \nabla \chi(\mathbf{r}, \omega)+i \omega \chi(\mathbf{r}, \omega)\} .
$$

This equation can be rewritten in the more convenient form

$$
\begin{aligned}
\Delta h_{I} & =e\left\{-i \frac{c}{\hbar} \boldsymbol{\alpha} \cdot \boldsymbol{p} \chi+i \omega \chi\right\} \\
& =-i \frac{e}{\hbar}\{[h, \chi]-\hbar \omega \chi\},
\end{aligned}
$$

where $h$ is the one-electron Dirac Hamiltonian given in Eq.(3). The requirement that matrix elements be independent of the gauge of the external field can be written,

$$
\left\langle b\left|\Delta h_{I}\right| a\right\rangle=-i \frac{e}{\hbar}\langle b|[h, \chi]-\hbar \omega \chi| a\rangle=-i \frac{e}{\hbar}\left(\epsilon_{b}-\epsilon_{a}-\hbar \omega\right)\langle b|\chi| a\rangle=0 .
$$

This condition is obviously satisfied for states $|a\rangle$ and $|b\rangle$ that satisfy $\hbar \omega=\epsilon_{b}-\epsilon_{a}$. Therefore, transition amplitudes calculated using single-particle orbitals in a local potential are gauge invariant, provided the energy of the incoming photon equals the difference in energies of the final and initial orbitals. Later, we require matrix elements of derivatives of $\Delta h_{I}$ with respect to $\omega$. These matrix elements can be simplified with the aid of the above identity to give

$$
\left\langle b\left|\frac{d \Delta h_{I}}{d \omega}\right| a\right\rangle=-i \frac{e}{\hbar}\left\langle b\left|\left[h, \frac{d \chi}{d \omega}\right]-\hbar \omega \frac{d \chi}{d \omega}-\hbar \chi\right| a\right\rangle=i e\langle b|\chi| a\rangle,
$$

where the equality on the right-hand side is valid only for states satisfying $\hbar \omega=\epsilon_{b}-\epsilon_{a}$.

The electromagnetic interaction of a many-electron system is just the sum of the interactions of the individual electrons given above. In second quantization, the electromagnetic interaction Hamiltonian is given by

$$
H_{I}(\omega)=\sum_{i j}\left(h_{I}\right)_{i j} a_{i}^{\dagger} a_{j}
$$

where $\left(h_{I}\right)_{i j}=\left\langle i\left|h_{I}(\mathbf{r}, \omega)\right| j\right\rangle$.

\subsection{Multipole potentials and transition amplitudes}

The vector and scalar potential for a photon in the transverse gauge are

$$
\begin{aligned}
\mathbf{A}(\mathbf{r}, \omega) & =\hat{\boldsymbol{\epsilon}} e^{i \mathbf{k} \cdot \mathbf{r}} \\
\phi(\mathbf{r}, \omega) & =0
\end{aligned}
$$

where $\hat{\boldsymbol{\epsilon}}$ is the photon's polarization vector and $\mathbf{k}$ is its wave vector. To simplify the calculation of transition amplitudes, we expand $\mathbf{A}(\mathbf{r}, \omega)$ in a multipole series

$$
\mathbf{A}(\mathbf{r}, \omega)=4 \pi \sum_{J M \lambda} i^{J-\lambda} \mathbf{Y}_{J M}^{(\lambda)}(\hat{k}) \cdot \hat{\boldsymbol{\epsilon}} \mathbf{a}_{J M}^{(\lambda)}(\mathbf{r})
$$


where the vector spherical harmonics, $\mathbf{Y}_{J M}^{(\lambda)}(\hat{k})$, are those defined in Akhiezer and Berestetskii [12]. The multipole components of the vector potential are given in the transverse gauge by

$$
\begin{aligned}
\mathbf{a}_{J M}^{(0)}(\mathbf{r})= & j_{J}(k r) \mathbf{Y}_{J M}^{(0)}(\hat{r}), \\
\mathbf{a}_{J M}^{(1)}(\mathbf{r})= & {\left[j_{J}^{\prime}(k r)+\frac{j_{J}(k r)}{k r}\right] \mathbf{Y}_{J M}^{(1)}(\hat{r}) } \\
& +\sqrt{J(J+1)} \frac{j_{J}(k r)}{k r} \mathbf{Y}_{J M}^{(-1)}(\hat{r}) .
\end{aligned}
$$

In these equations, $\lambda=0$ designates magnetic multipoles and $\lambda=1$ designates electric multipoles. The parity of the multipole potential $\mathbf{a}_{J M}^{(\lambda)}(\mathbf{r})$ is $(-1)^{J+1-\lambda}$. Matrix elements of the electric-dipole interaction in the transverse gauge reduce to velocity-form dipole amplitudes in the nonrelativistic limit. Later we refer to the transverse-gauge amplitudes as velocity-form amplitudes.

Gauge transformations, Eqs. $(23,24)$, have no effect on the magnetic-multipole potentials, but they do modify electric-multipole potentials. In particular, a gauge transformation of the transverse-gauge potentials using the gauge function,

$$
\chi_{J M}(\mathbf{r}, \omega)=-\frac{1}{k} \sqrt{\frac{J+1}{J}} j_{J}(k r) Y_{J M}(\hat{r}),
$$

gives transition amplitudes that reduce to length form in the nonrelativistic limit. We refer to the resulting potentials as length-gauge potentials in the sequel. The electric-multipole potentials in the length gauge are

$$
\begin{aligned}
\mathbf{a}_{J M}^{(1)}(\mathbf{r}) & =-j_{J+1}(k r)\left[\mathbf{Y}_{J M}^{(1)}(\hat{r})-\sqrt{\frac{J+1}{J}} \mathbf{Y}_{J M}^{(-1)}(\hat{r})\right], \\
\phi_{J M}^{(1)}(k r) & =-i c \sqrt{\frac{J+1}{J}} j_{J}(k r) Y_{J M}(\hat{r}) .
\end{aligned}
$$

In either gauge, the multipole-interaction Hamiltonian can be written in terms of dimensionless multipole-transition operators $t_{J M}^{(\lambda)}(\mathbf{r}, \omega)$

$$
\left[h_{I}(\mathbf{r}, \omega)\right]_{J M}=-i e c \sqrt{\frac{(2 J+1)(J+1)}{4 \pi J}} t_{J M}^{(\lambda)}(\mathbf{r}, \omega),
$$

where the one-electron reduced matrix elements $\left\langle i\left\|t_{J}^{(\lambda)}\right\| j\right\rangle$ are Transverse Gauge:

$$
\left\langle\kappa_{i}\left\|t_{J}^{(0)}\right\| \kappa_{j}\right\rangle=\left\langle-\kappa_{i}\left\|C_{J}\right\| \kappa_{j}\right\rangle \int_{0}^{\infty} d r \frac{\kappa_{i}+\kappa_{j}}{J+1} j_{J}(k r)\left[G_{i}(r) F_{j}(r)+F_{i}(r) G_{j}(r)\right],
$$




$$
\begin{aligned}
& \left\langle\kappa_{i}\left\|t_{J}^{(1)}\right\| \kappa_{j}\right\rangle=\left\langle\kappa_{i}\left\|C_{J}\right\| \kappa_{j}\right\rangle \int_{0}^{\infty} d r\left\{-\frac{\kappa_{i}-\kappa_{j}}{J+1}\left[j_{J}^{\prime}(k r)+\frac{j_{J}(k r)}{k r}\right]\right. \\
& \left.\times\left[G_{i}(r) F_{j}(r)+F_{i}(r) G_{j}(r)\right]+J \frac{j_{J}(k r)}{k r}\left[G_{i}(r) F_{j}(r)-F_{i}(r) G_{j}(r)\right]\right\},
\end{aligned}
$$

and

Length Gauge:

$$
\begin{gathered}
\left\langle\kappa_{i}\left\|t_{J}^{(1)}\right\| \kappa_{j}\right\rangle=\left\langle\kappa_{i}\left\|C_{J}\right\| \kappa_{j}\right\rangle \int_{0}^{\infty} d r\left\{j_{J}(k r)\left[G_{i}(r) G_{j}(r)+F_{i}(r) F_{j}(r)\right]\right. \\
\left.j_{J+1}(k r)\left[\frac{\kappa_{i}-\kappa_{j}}{J+1}\left[G_{i}(r) F_{j}(r)+F_{i}(r) G_{j}(r)\right]+\left[G_{i}(r) F_{j}(r)-F_{i}(r) G_{j}(r)\right]\right]\right\} .
\end{gathered}
$$

In Eqs. $(38,39)$, the functions $G_{i}(r)$ and $F_{i}(r)$ are the large and small components, respectively, of the radial Dirac wave functions for the orbital with quantum numbers $\left(n_{i}, \kappa_{i}\right)$.

The multipole-transition operators $t_{J}^{(\lambda)}(\mathbf{r}, \omega)$ are related to the frequencydependent multipole-moment operators $q_{J}^{(\lambda)}(\mathbf{r}, \omega)$ by

$$
q_{J}^{(\lambda)}(\mathbf{r}, \omega)=\frac{(2 J+1) ! !}{k^{J}} t_{J}^{(\lambda)}(\mathbf{r}, \omega) .
$$

Both the transition operators and the multipole-moment operators are irreducible tensor operators. For a many-body system, the multipole-transition operators are given by

$$
T_{J M}^{(\lambda)}=\sum_{i j}\left(t_{J M}^{(\lambda)}\right)_{i j} a_{i}^{\dagger} a_{j},
$$

where $\left(t_{J M}^{(\lambda)}\right)_{i j}=\left\langle i\left|t_{J M}^{(\lambda)}(\mathbf{r}, \omega)\right| j\right\rangle$. The Einstein $B$ coefficient, giving the absorption probability per unit time of a photon of multipolarity $(J \lambda)$ leading from a state $I$ with angular momentum $J_{I}$, to a state $F$ with angular momentum $J_{F}$, is

$$
B_{J}^{(\lambda)}=2 \alpha \omega \frac{[J]}{\left[J_{I}\right]} \frac{J+1}{J}\left|\left\langle F|| T_{J}^{(\lambda)}|| I\right\rangle\right|^{2}=8 \pi k \frac{[J]}{\left[J_{I}\right]} \frac{J+1}{J}\left|\left\langle F|| T_{J}^{(\lambda)}|| I\right\rangle\right|^{2} c R_{\infty},
$$

where we use the notation $[J]=2 J+1$. The corresponding Einstein A coefficient, giving the spontaneous emission probability for transitions from state $I$ to state $F$ with $E_{I}-E_{F}=\hbar \omega$ is, of course, given by

$$
A_{J}^{(\lambda)}=B_{J}^{(\lambda)} .
$$

Using wave functions given in Eq.(12) for both the initial and final states 
and carrying out the sums over magnetic substates, one obtains

$$
\begin{aligned}
& \left\langle F\left\|T_{J}^{(\lambda)}\right\| I\right\rangle=\sqrt{\left[J_{I}\right]\left[J_{F}\right]}(-1)^{J} \sum_{\substack{m \leq n \\
r \leq s}} \eta_{r s} \eta_{m n} c_{r s}^{(F)} c_{m n}^{(I)} \times \\
& \left\{(-1)^{j_{r}+j_{s}+J_{I}}\left\{\begin{array}{ccc}
J & J_{I} & J_{F} \\
j_{s} & j_{r} & j_{m}
\end{array}\right\}\left\langle r\left\|t^{(\lambda)}\right\| m\right\rangle \delta_{n s}+(-1)^{j_{r}+j_{n}}\left\{\begin{array}{ccc}
J & J_{I} & J_{F} \\
j_{s} & j_{r} & j_{n}
\end{array}\right\}\left\langle r\left\|t^{(\lambda)}\right\| n\right\rangle \delta_{m s}\right. \\
& \left.+(-1)^{J_{F}+J_{I}+1}\left\{\begin{array}{ccc}
J & J_{I} & J_{F} \\
j_{r} & j_{s} & j_{m}
\end{array}\right\}\left\langle s\left\|t^{(\lambda)}\right\| m\right\rangle \delta_{n r}+(-1)^{j_{r}+j_{n}+J_{F}}\left\{\begin{array}{ccc}
J & J_{I} & J_{F} \\
j_{r} & j_{s} & j_{n}
\end{array}\right\}\left\langle s\left\|t^{(\lambda)}\right\| n\right\rangle \delta_{m r}\right\} .
\end{aligned}
$$

Equations(42-44) are used in the following sections to evaluate multipole-transition amplitudes and rates.

\subsection{A commutator identity}

For electric multipole transitions $(\lambda=1)$, we consider the difference between the transition amplitudes in length gauge $\left(T_{J M}^{(1)}\right)_{l}$ and in velocity gauge $\left(T_{J M}^{(1)}\right)_{v}$. Let us introduce the operator representing the difference between the multipoletransition operators in the two gauges

$$
\Delta T_{J M}^{(1)}=\left(T_{J M}^{(1)}\right)_{l}-\left(T_{J M}^{(1)}\right)_{v} .
$$

This difference can be written in terms of the multipole-interaction Hamiltonian using Eq.(36):

$$
\Delta T_{J M}^{(1)}=\frac{i}{e c} \sqrt{\frac{4 \pi J}{(J+1)(2 J+1)}} \Delta H_{J M},
$$

where

$$
\Delta H_{J M}=\sum_{i j}\left\langle i\left|\left(\Delta h_{I}\right)_{J M}\right| j\right\rangle a_{i}^{\dagger} a_{j}
$$

Equation (46) can be rewritten with the aid of Eq.(28) to give

$$
\begin{aligned}
\Delta H_{J M} & =-i \frac{e}{\hbar}\left\{\left[H_{0}, X_{J M}\right]-\hbar \omega X_{J M}\right\} \\
& =-i \frac{e}{\hbar}\left\{\left[H, X_{J M}\right]-\hbar \omega X_{J M}-\left[V, X_{J M}\right]\right\},
\end{aligned}
$$

where $V=V_{C}+V_{B}$ is the sum of the Coulomb and Breit interactions, and where

$$
X_{J M}=\sum_{i j}\left(\chi_{J M}\right)_{i j} a_{i}^{\dagger} a_{j}
$$

is the many-electron gauge operator. For eigenstates of $H$ that satisfy $E_{F}-E_{I}=$ $\hbar \omega$, it follows from Eq.(47) that

$$
\left\langle\Psi_{F}\left|\Delta H_{J M}\right| \Psi_{I}\right\rangle=i \frac{e}{\hbar}\left\langle\Psi_{F}\left|\left[V, X_{J M}\right]\right| \Psi_{I}\right\rangle .
$$


Using Eq.(34) and Eq.(46), we can formulate Eq.(48) as the commutator identity

$$
\left\langle\Psi_{F}\left|\Delta T_{J M}\right| \Psi_{1}\right\rangle=\frac{1}{\hbar \omega}\left\langle\Psi_{F}\left|\left[V, \Xi_{J M}\right]\right| \Psi_{I}\right\rangle,
$$

where

$$
\Xi_{J M}=\sum_{i j}\left(\xi_{J M}\right)_{i j} a_{i}^{\dagger} a_{j}
$$

with

$$
\xi_{J M}(\mathbf{r}, \omega)=\sqrt{\frac{4 \pi}{2 J+1}} j_{J}(k r) Y_{J M}(\hat{r}) .
$$

Since the no-pair potential $V$ in Eq.(49) contains only positive-energy components, the commutator of $V$ and $\Xi_{J M}$ does not vanish. Using the wave functions given in Eq.(12) for the case $U(r)=0$, the reduced matrix element of $\left[V, \Xi_{J M}\right]$ is found to be

$$
\begin{gathered}
\left\langle F||\left[V, \Xi_{J}\right] \| I\right\rangle=\sqrt{\left[J_{F}\right]\left[J_{I}\right]} \sum_{\substack{m \leq n \\
r \leq s}} \eta_{r s} \eta_{m n} c_{r s}^{(F)} c_{m n}^{(I)} \times \\
\sum_{i, L}\left[(-1)^{j_{n}+j_{s}+J_{F}+J_{I}+L}\left\{\begin{array}{ccc}
j_{i} & j_{n} & J_{F} \\
j_{s} & j_{r} & L
\end{array}\right\}\left\{\begin{array}{ccc}
j_{i} & j_{n} & J_{F} \\
J_{I} & J & j_{m}
\end{array}\right\} X_{L}(\text { srni })\left\langle i\left\|\xi_{J}\right\| m\right\rangle\right. \\
+(-1)^{j_{n}+j_{s}+J_{I}+L}\left\{\begin{array}{ccc}
j_{i} & j_{n} & J_{F} \\
j_{r} & j_{s} & L
\end{array}\right\}\left\{\begin{array}{ccc}
j_{i} & j_{n} & J_{F} \\
J_{I} & J & j_{m}
\end{array}\right\} X_{L}(r s n i)\left\langle i\left\|\xi_{J}\right\| m\right\rangle \\
+(-1)^{j_{n}+j_{s}+J_{F}+L}\left\{\begin{array}{ccc}
j_{i} & j_{m} & J_{F} \\
j_{s} & j_{r} & L
\end{array}\right\}\left\{\begin{array}{ccc}
j_{i} & j_{m} & J_{F} \\
J_{I} & J & j_{n}
\end{array}\right\} X_{L}(\text { srmi })\left\langle i\left\|\xi_{J}\right\| n\right\rangle \\
+(-1)^{j_{n}+j_{s}+L}\left\{\begin{array}{ccc}
j_{i} & j_{m} & J_{F} \\
j_{r} & j_{s} & L
\end{array}\right\}\left\{\begin{array}{ccc}
j_{i} & j_{m} & J_{F} \\
J_{I} & J & j_{n}
\end{array}\right\} X_{L}(r s m i)\left\langle i\left\|\xi_{J}\right\| n\right\rangle \\
+(-1)^{j_{m}-j_{r}+L}\left\{\begin{array}{ccc}
j_{i} & j_{s} & J_{I} \\
j_{n} & j_{m} & L
\end{array}\right\}\left\{\begin{array}{ccc}
j_{i} & j_{s} & J_{I} \\
J_{F} & J & j_{r}
\end{array}\right\}\left\langle r\left\|\xi_{J}\right\| i\right\rangle X_{L}(i s m n) \\
+(-1)^{j_{m}-j_{r}+J_{I}+L}\left\{\begin{array}{ccc}
j_{i} & j_{s} & J_{I} \\
j_{m} & j_{n} & L
\end{array}\right\}\left\{\begin{array}{ccc}
j_{i} & j_{s} & J_{I} \\
J_{F} & J & j_{r}
\end{array}\right\}\left\langle r\left\|\xi_{J}\right\| i\right\rangle X_{L}(\text { isnm }) \\
+(-1)^{j_{m}-j_{r}+J_{F}+L}\left\{\begin{array}{cccc}
j_{i} & j_{r} & J_{I} \\
j_{n} & j_{m} & L
\end{array}\right\}\left\{\begin{array}{ccc}
j_{i} & j_{r} & J_{I} \\
J_{F} & J & j_{s}
\end{array}\right\}\left\langle s\left\|\xi_{J}\right\| i\right\rangle X_{L}(\text { irmn }) \\
\left.+(-1)^{j_{m}-j_{r}+J_{F}+J_{I}+L}\left\{\begin{array}{lll}
j_{i} & j_{r} & J_{I} \\
j_{m} & j_{n} & L
\end{array}\right\}\left\{\begin{array}{ccc}
j_{i} & j_{r} & J_{I} \\
J_{F} & J & j_{s}
\end{array}\right\}\left\langle s\left\|\xi_{J}\right\| i\right\rangle X_{L}(\text { irnm })\right] .
\end{gathered}
$$

From the definition of the no-pair potential, it follows that the sum over $i$ in Eq.(50) is restricted to positive-energy single-particle orbitals. Thus, electromagnetic transition amplitudes calculated using the no-pair Hamiltonian are gauge dependent. If we artificially extend the sum over $i$ to include the complete set of positive- and negative-energy states, then, as is shown in the Appendix, the commutator vanishes identically. 
Nonvanishing values are a result of the restriction to positive-energy states in the no-pair Hamiltonian. In Subsection 2.5, we calculate reduced matrix elements of the E1 transition operator for heliumlike ions in length and velocity gauges as well as the reduced matrix element of the commutator from Eq.(50). The length-velocity difference is found to be equal to the matrix-element of the commutator to the numerical accuracy of the calculation.

\subsection{Numerical calculations and tests of transition ampli- tudes}

To determine the transition amplitudes numerically, we first solve the CI equations (17) either iteratively [4] or directly as a large-scale eigenvalue problem [3]. After obtaining wave functions and energies, we evaluate the reduced matrix elements of the transition amplitudes using Eq.(44). For concreteness, we consider only calculations carried out starting from the potential $U(r)=0$ in this subsection.

In our numerical calculations, we set the goal of obtaining amplitudes accurate to five significant figures. Since it is relatively expensive and time consuming to carry out the multiple sums in Eq.(44), we limit our basis sets as much as possible, within this accuracy constraint. For the allowed E1 transitions $2{ }^{1} P_{1} \rightarrow 1{ }^{1} S_{0}$ and $2{ }^{3} P_{0,1,2} \rightarrow 2{ }^{3} S_{1}$, as well as the $2{ }^{3} S_{1} \rightarrow 1{ }^{1} S_{0}$ (M1) and $2^{3} P_{2} \rightarrow 1{ }^{1} S_{0}$ (M2) transitions, we found that the following restrictions on basis sets gave amplitudes of five-figure accuracy. For nuclear charges in the range $Z=2-10$, the basis orbitals ( $\mathrm{nmrs}$ ) in Eq.(44) were limited to those having orbital angular momentum $l \leq 4$. For $Z=11-30$, it was sufficient to include orbitals with $l \leq 3$; for $Z>30$ only those orbitals with $l \leq 2$ were required. For $Z \leq 30$, we summed only the first 20 out of a set of 40 basis orbitals for each $l$ value considered. For $Z>30$, it was necessary to sum the first 25 orbitals from a set of 40 for each $l$.

In Table 1, we compare amplitudes evaluated with the limited basis sets described above with those obtained using complete basis sets [3] for nuclear charges $Z=10,20,30$ and 40 . For this comparison, the Breit interaction was omitted entirely from the calculation. As seen from this table, the difference between the truncated and exact amplitudes is at worse a few parts in the fifth significant digit. For the forbidden E1 transitions $2{ }^{3} P_{1} \rightarrow{ }^{1} S_{0}$ and $2{ }^{1} P_{1} \rightarrow$ $1^{2} S_{1}$, the basis sets described above were too small to give five-figure accuracy $Z<50$. For $Z \leq 30$, we needed orbitals with $l \leq 6$, while for $31 \leq Z \leq 40$, we needed orbitals with $l \leq 4$, and for $41 \leq Z \leq 49$, orbitals with $l \leq 3$ were required. For all $Z$, we summed over 25 of 40 basis functions.

To facilitate comparisons of values in Table 1 and in the other tables in this paper with pervious calculations, we factor $k^{J} /(2 J+1)$ !! from the transition amplitudes to obtain the multipole-moment operators $Q_{J M}^{(\lambda)}$,

$$
Q_{J M}^{(\lambda)}=\frac{(2 J+1) ! !}{k^{J}} T_{J M}^{(\lambda)}
$$


Table 1: Comparison of calculations of reduced matrix elements using the limited number of configurations described in the text with complete CI calculations. Results do not include Breit corrections to the wave function.

\begin{tabular}{|c|c|c|c|c|}
\hline$Z$ & Transition & $\begin{array}{c}\text { Limited } \\
\text { Configurations }\end{array}$ & $\begin{array}{c}\text { Complete } \\
\text { Configurations }\end{array}$ & $\begin{array}{c}\text { Percent } \\
\text { Difference }\end{array}$ \\
\hline \multirow[t]{9}{*}{10} & $2{ }^{1} P_{1}-1{ }^{1} S_{0}$ & 0.178458 & 0.178461 & $1.8(-3)$ \\
\hline & $2{ }^{1} P_{1}-2{ }^{1} S_{0}$ & 0.577485 & 0.577483 & $4.1(-4)$ \\
\hline & $2{ }^{1} P_{1}-2{ }^{3} S_{1}$ & 0.012193 & 0.012195 & $1.6(-2)$ \\
\hline & $2^{3} P_{1}-1^{1} S_{0}$ & 0.003913 & 0.003913 & $1.6(-2)$ \\
\hline & $2^{3} P_{0}-2^{3} S_{1}$ & 0.324405 & 0.324405 & $1.3(-4)$ \\
\hline & $2^{3} P_{1}-2^{3} S_{1}$ & 0.561963 & 0.561962 & $1.3(-4)$ \\
\hline & $2^{3} P_{2}-2^{3} S_{1}$ & 0.726198 & 0.726198 & $1.3(-4)$ \\
\hline & $2^{3} S_{1}-1^{1} S_{0}$ & 0.001767 & 0.001767 & $1.8(-3)$ \\
\hline & $2^{3} P_{2}-1^{1} S_{0}$ & 0.881752 & 0.881737 & $1.6(-3)$ \\
\hline \multirow[t]{9}{*}{20} & $2{ }^{1} P_{1}-1{ }^{1} S_{0}$ & 0.088423 & 0.088422 & $1.4(-3)$ \\
\hline & $2{ }^{1} P_{1}-2{ }^{1} S_{0}$ & 0.269181 & 0.269179 & $8.7(-4)$ \\
\hline & $2{ }^{1} P_{1}-2^{3} S_{1}$ & 0.041923 & 0.041932 & $2.1(-2)$ \\
\hline & $2^{3} P_{1}-1^{1} S_{0}$ & 0.014052 & 0.014055 & $2.1(-2)$ \\
\hline & $2^{3} P_{0}-2^{3} S_{1}$ & 0.154662 & 0.154662 & $8.7(-5)$ \\
\hline & $2^{3} P_{1}-2^{3} S_{1}$ & 0.265030 & 0.265025 & $1.7(-3)$ \\
\hline & $2{ }^{3} P_{2}-2^{3} S_{1}$ & 0.347534 & 0.347534 & $6.9(-5)$ \\
\hline & $2{ }^{3} S_{1}-1^{1} S_{0}$ & 0.007208 & 0.007208 & $6.5(-4)$ \\
\hline & $2^{3} P_{2}-1^{1} S_{0}$ & 0.432032 & 0.432027 & $1.0(-3)$ \\
\hline \multirow[t]{9}{*}{30} & $2{ }^{1} P_{1}-{ }^{1} S_{0}$ & 0.055224 & 0.055221 & $4.8(-3)$ \\
\hline & $2{ }^{1} P_{1}-2^{1} S_{0}$ & 0.166329 & 0.166325 & $2.2(-3)$ \\
\hline & $2{ }^{1} P_{1}-2{ }^{3} S_{1}$ & 0.062073 & 0.062082 & $1.4(-2)$ \\
\hline & $2^{3} P_{1}-1^{1} S_{0}$ & 0.021048 & 0.021051 & $1.4(-2)$ \\
\hline & $2^{3} P_{0}-2^{3} S_{1}$ & 0.100630 & 0.100630 & $6.0(-5)$ \\
\hline & $2^{3} P_{1}-2^{3} S_{1}$ & 0.163590 & 0.163584 & $3.4(-3)$ \\
\hline & $2^{3} P_{2}-2^{3} S_{1}$ & 0.227606 & 0.227606 & $4.7(-5)$ \\
\hline & $2^{3} S_{1}-1^{1} S_{0}$ & 0.016392 & 0.016392 & $5.7(-4)$ \\
\hline & $2^{3} P_{2}-1^{1} S_{0}$ & 0.282034 & 0.282032 & $6.5(-4)$ \\
\hline \multirow[t]{9}{*}{40} & $2{ }^{1} P_{1}-1^{1} S_{0}$ & 0.038251 & 0.038248 & $6.4(-3)$ \\
\hline & $2^{1} P_{1}-2^{1} S_{0}$ & 0.115910 & 0.115908 & $2.1(-3)$ \\
\hline & $2{ }^{1} P_{1}-2{ }^{3} S_{1}$ & 0.061313 & 0.061317 & $6.9(-3)$ \\
\hline & $2^{3} P_{1}-1^{1} S_{0}$ & 0.020851 & 0.020852 & $7.1(-3)$ \\
\hline & $2^{3} P_{0}-2^{3} S_{1}$ & 0.073786 & 0.073786 & $1.1(-4)$ \\
\hline & $2{ }^{3} P_{1}-2^{3} S_{1}$ & 0.113168 & 0.113161 & $6.8(-3)$ \\
\hline & $2^{3} P_{2}-2^{3} S_{1}$ & 0.168470 & 0.168469 & $6.9(-5)$ \\
\hline & $2^{3} S_{1}-1^{1} S_{0}$ & 0.029440 & 0.029440 & $3.2(-4)$ \\
\hline & $2^{3} P_{2}-1^{1} S_{0}$ & 0.205929 & 0.205929 & 0 \\
\hline
\end{tabular}


For E1 transitions, we tabulate reduced matrix elements of $Q_{1 M}^{(1)}$, while for M1 and M2 transitions, we tabulate reduced matrix elements of the magneticmultipole operator

$$
M_{J M}=2 c Q_{J M}^{(0)} .
$$

It should be noted that the magnetic-quadrupole amplitude defined in this way is the conventional moment given in many textbooks [13]. It differs from that used in some compilations of transition amplitudes [14] by a factor of $3 / 2$.

Tests of the commutator identity are shown in Table 2 , where values of the reduced matrix elements of $Q_{1 M}^{(1)}$ are given for ions with $Z=20,50$, and 100 . As can be seen by comparing the length-form and velocity-form amplitudes in the third and fourth columns of the table, the amplitudes differ in the two gauges. The differences, which are tabulated in the fifth column of the table, are typically in the fourth significant figure, though differences occur in the second figure for the $2{ }^{3} P_{0,1} \rightarrow 2{ }^{3} S_{1}$ transitions at high $Z$. To convince ourselves that these differences are of a physical origin, we compare them with the values predicted by the commutator identity Eq.(49), which are listed in the last column of the table. The equality of the entries in the last two columns confirms that the length-velocity differences in the numerical amplitudes are just those expected for calculations carried-out using the no-pair theory.

It should be mentioned that the multiple sums in the expression for the commutator are also evaluated using a reduced basis set. We restrict the orbitals ( $n m r s)$ in Eq.(50) to include those having angular momentum quantum number $l \leq 2$. We further restrict the orbitals by summing only the first 8 states in a basis set of 40 orbitals. The sums over $L$ are also restricted to values $L \leq 2$, while the sum over $i$ includes all 40 positive-energy basis functions and all values of angular momentum permitted by the selection rules. Because of the large number of indices, these sums are time consuming. Nevertheless, in selected cases, we verify that on increasing the limits above sufficiently, the differences in the transition amplitudes agree to five figures with the values predicted by the commutator identity. As a further check on our numerical methods, we extend the sum over $i$ to include 40 positive-energy orbitals and 40 negative-energy orbitals and find that the reduced matrix element of the commutator vanishes to more than six figures for each of the cases considered in Table 2.

From the values in Table 2, it is clear that transition amplitudes determined from the no-pair Hamiltonian are gauge dependent, and that the gauge dependence is a result of excluding negative-energy states in the no-pair Hamiltonian. In the following section, we examine electromagnetic transitions in hydrogenlike ions in a gauge-invariant perturbation theory calculation based on QED. This analysis will be used in Sec. 3 to construct gauge-invariant amplitudes for heliumlike ions in perturbation theory. 
Table 2: Reduced matrix elements of the electric-dipole operator in both the length and velocity gauges obtained using CI wave functions from the BreitCoulomb, no-pair Hamiltonian. $\Delta Q$ is the difference between the matrix elements in the two gauges, the final column $[V, \Xi]$ gives values of the difference predicted by the commutator identity Eq.(49). Units: a.u.

\begin{tabular}{cccccc}
\hline \hline$Z$ & Transition & $\left\langle F\left\|Q_{1}\right\| I\right\rangle_{l}$ & $\left\langle F\left\|Q_{1}\right\| I\right\rangle_{v}$ & \multicolumn{1}{c}{$\Delta Q$} & \multicolumn{1}{c}{$[V, \Xi]$} \\
\hline 20 & $2{ }^{1} P_{1}-1{ }^{1} S_{0}$ & 0.088563 & 0.088594 & -0.000031 & -0.000033 \\
& $2{ }^{3} P_{1}-1{ }^{1} S_{0}$ & 0.014967 & 0.014972 & -0.000005 & -0.000005 \\
& $2{ }^{1} P_{1}-2{ }^{3} S_{1}$ & 0.045270 & 0.045221 & 0.000049 & 0.000048 \\
& $2{ }^{3} P_{0}-2{ }^{3} S_{1}$ & 0.154748 & 0.154398 & 0.000350 & 0.000360 \\
& $2{ }^{3} P_{1}-2{ }^{3} S_{1}$ & 0.264555 & 0.264030 & 0.000525 & 0.000537 \\
& $2{ }^{3} P_{2}-2{ }^{3} S_{1}$ & 0.347554 & 0.347051 & 0.000503 & 0.000514 \\
50 & $2{ }^{1} P_{1}-1{ }^{1} S_{0}$ & 0.029263 & 0.029291 & -0.000028 & -0.000028 \\
& $2{ }^{3} P_{1}-1{ }^{1} S_{0}$ & 0.018526 & 0.018542 & -0.000016 & -0.000017 \\
& $2{ }^{1} P_{1}-2{ }^{3} S_{1}$ & 0.055209 & 0.055072 & 0.000137 & 0.000140 \\
& $2{ }^{3} P_{0}-2{ }^{3} S_{1}$ & 0.057633 & 0.056537 & 0.001097 & 0.001106 \\
& $2{ }^{3} P_{1}-2{ }^{3} S_{1}$ & 0.084451 & 0.083239 & 0.001212 & 0.001228 \\
& $2{ }^{3} P_{2}-2{ }^{3} S_{1}$ & 0.133095 & 0.132905 & 0.000190 & 0.000192 \\
& & & & & \\
100 & $2{ }^{1} P_{1}-1{ }^{1} S_{0}$ & 0.011478 & 0.011499 & -0.000021 & -0.000021 \\
& $2{ }^{3} P_{1}-1{ }^{1} S_{0}$ & 0.008181 & 0.008193 & -0.000012 & -0.000012 \\
& $2{ }^{1} P_{1}-2{ }^{3} S_{1}$ & 0.026731 & 0.026689 & 0.000042 & 0.000042 \\
& $2{ }^{3} P_{0}-2{ }^{3} S_{1}$ & 0.022804 & 0.020437 & 0.002367 & 0.002394 \\
& $2{ }^{3} P_{1}-2{ }^{3} S_{1}$ & 0.032173 & 0.028439 & 0.003734 & 0.003757 \\
& $2{ }^{3} P_{2}-2{ }^{3} S_{1}$ & 0.059723 & 0.059725 & -0.000002 & -0.000001 \\
\hline \hline
\end{tabular}




\section{$3 \quad S$-Matrix Theory for Decay Rates}

Although we are interested in heliumlike ions, in this section we consider the simpler problem of obtaining gauge-independent transition rates for one-electron ions in an external potential, which is chosen to be a Coulomb potential of charge $Z_{0}$ together with a perturbing potential $V(r)$. We use the resulting theoretical analysis as a paradigm for constructing gauge-invariant amplitudes for transitions in heliumlike ions. To investigate the transition amplitudes in a gaugeinvariant framework, we turn to the QED $S$-matrix. Analysis of transitions from the point of view of QED shows that transition amplitudes are given by the usual Feynman diagrams from perturbation theory, together with derivative terms that have no diagrammatic representation. We use this example primarily to elucidate the structure of such derivative terms.

Specifically, we will work with an interaction Hamiltonian $H_{I}=H_{I}^{A}+H_{I}^{B}$, where

$$
H_{I}^{A}=e \int d^{3} r \bar{\psi}(r) \gamma_{\mu} A^{\mu}(r) \psi(r)
$$

and

$$
H_{I}^{B}=\int d^{3} r \bar{\psi}(r) V(r) \psi(r)
$$

Here $V(r)$ represents an external potential that is in principle arbitrary. One choice that illustrates the important features of the theory is

$$
V(r)=-\frac{\delta Z}{r} \gamma_{0}
$$

Here $\delta Z \equiv Z-Z_{0}$, where $Z$ is the actual charge of the nucleus of a hydrogenlike ion and $Z_{0}$ a nearby charge that defines the potential in which the calculation is carried out. For example, to calculate decay rates of hydrogenlike thallium $(Z=81)$ as perturbations of the decay rate of hydrogenlike mercury $(Z=80)$, we would have $\delta Z=1$. Since both decay rates are known, the difference must be accounted for dominantly by first-order perturbation theory. Another choice of $V(r)$ has been described by Indelicato and Mohr [15], which allows energy levels of lithiumlike uranium to be calculated as perturbations from hydrogenlike uranium with $V(r)$ coming from the potential of the two $1 s$ electrons.

In order to calculate decay rates, we recall the well-known fact that these rates are related to energy level shifts $\Delta E$ by $\Gamma=-2 \operatorname{Im} \Delta E$. This approach was used by Barbieri and Sucher [6] in a paper closely related to the present work to calculate radiative corrections to M1 decays in hydrogenlike ions. In order to calculate $\Delta E$, we use $S$-matrix techniques. The basic equation used is the symmetrized version of the Gell-Mann-Low formula [7] given by Sucher [16],

$$
\Delta E_{n}=\frac{i \epsilon}{2} \lim _{\epsilon \rightarrow 0} \frac{\partial}{\partial \lambda} \ln \left\langle n\left|S_{\epsilon, \lambda}\right| n\right\rangle
$$

where

$$
S_{\epsilon, \lambda}=e^{-i \lambda \int d^{4} x e^{-\epsilon\left|x_{0}\right|} H_{I}(x)}
$$




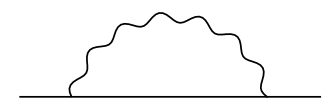

Figure 1: Feynman diagram for $\Sigma_{v v}(E)$. The imaginary part of this diagram gives the leading contribution to the decay rate.

We apply these formulas to a hydrogenic ion with an electron in state $v$. The lowest-order contributions to the $S$-matrix come from a single action of $H_{I}^{B}$,

$$
S_{\epsilon, \lambda}^{(1)}=-\frac{2 i \lambda}{\epsilon} E^{(1)}(v)
$$

and two actions of $H_{I}^{A}$,

$$
S_{\epsilon, \lambda}^{(2)}=-\frac{i \lambda^{2}}{\epsilon} \Sigma_{v v}\left(\epsilon_{v}\right)
$$

Here we have introduced

$$
E^{(1)}(v)=\int d^{3} r \bar{\psi}_{v}(r) V(r) \psi_{v}(r)
$$

and defined a generalization of the usual self-energy

$$
\Sigma_{m n}\left(\epsilon_{v}\right) \equiv-i \alpha \int d^{3} x d^{3} y \int \frac{d^{4} k}{(2 \pi)^{4}} \frac{e^{i \vec{k} \cdot(\vec{x}-\vec{y})}}{k^{2}+i \delta} \bar{\psi}_{m}(\vec{x}) \gamma_{\mu} S_{F}\left(\vec{x}, \vec{y} ; \epsilon_{v}-k_{0}\right) \gamma^{\mu} \psi_{n}(\vec{y})
$$

We ignore the other radiative correction associated with two actions of $H_{I}^{A}$ describing vacuum polarization because it is real.

We can now determine the lowest-order decay rate from the imaginary part of the Lamb shift, $\Sigma_{v v}\left(\epsilon_{v}\right)$ illustrated in Fig. 1. That imaginary part arises solely from the 'pole terms' [17], which arise when a Wick rotation $k_{0} \rightarrow i k_{4}$ is carried out, and result from the contour passing through poles associated with electrons of energy lower than that of the valence electron $v$, which we will refer to as $m$. Specifically, the pole terms are, in Feynman gauge,

$$
\Delta E_{\text {pole }}=\alpha \sum_{m<v} \int \frac{d^{3} x d^{3} y}{|\vec{x}-\vec{y}|} e^{i\left(\epsilon_{v}-\epsilon_{m}\right)|\vec{x}-\vec{y}|} \bar{\psi}_{v}(\vec{x}) \gamma_{\mu} \psi_{m}(\vec{x}) \bar{\psi}_{m}(\vec{y}) \gamma^{\mu} \psi_{v}(\vec{y}) .
$$

The real part of this contributes to the Lamb shift, and the imaginary part reproduces the lowest-order decay rate. Note there is also a half-pole term when $m=v$, which enters with a factor $1 / 2$, but is purely real. It is a simple matter to evaluate the imaginary part of this expression, and agreement with the standard result is found. Because the self-energy is gauge-invariant, the same rates must be obtained if the Coulomb gauge is used instead of the Feynman gauge. For decays of multipolarity $(J \lambda)$, we obtain

$$
\operatorname{Im} \Delta E(\omega)=-\alpha \omega \frac{2 J+1}{2 j_{v}+1} R^{2}(\omega)
$$




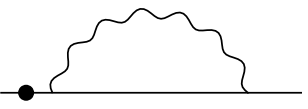

$(l)$

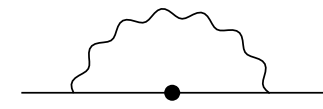

$(v)$

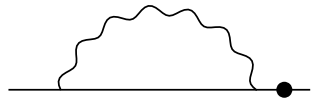

$(r)$

Figure 2: Feynman diagrams for the three contributions to the third-order $S$ matrix leading to the dominant correction to the decay rate. The solid dot $\bullet$ represents the interaction with the external field $V$. The labels $(l),(r)$, and $(v)$ represent left, right, and vertex, respectively.

where $\hbar \omega=\epsilon_{v}-\epsilon_{m}$, and where

$$
R(\omega)=\sqrt{\frac{J+1}{J}}\left\langle v\left\|t_{J}^{(\lambda)}\right\| m\right\rangle .
$$

The reduced matrix elements $\left\langle v\left\|t_{J}^{(\lambda)}\right\| m\right\rangle$ here are precisely those defined in (38-39). Equation (61) leads to a decay rate in agreement with the general expression given in Eq.(43).

We now consider corrections of higher order. We will be concerned with two actions of $H_{I}^{A}$ and one of $H_{I}^{B}$. A first contribution of this order comes from the lowest-order formulas for $S^{(1)}$ and $S^{(2)}$ given above, which give an energy shift that diverges in the limit $\epsilon \rightarrow 0$,

$$
\Delta E^{\operatorname{div}}=\frac{i}{\epsilon}\left(E^{(1)}(v)+\Sigma_{v v}\left(\epsilon_{v}\right)\right)\left(2 E^{(1)}(v)+\Sigma_{v v}\left(\epsilon_{v}\right)\right)
$$

We will be concerned here only with the contribution $3 i / \epsilon E^{(1)}(v) \Sigma_{v v}\left(\epsilon_{v}\right)$. It clearly must be canceled by a term from the third-order $S$ matrix, which we will show is connected with the derivative terms we are interested in. There are three contributions to the $S$-matrix. represented by the Feynman diagrams of Figs. $2(l),(v)$ and $(r)$. (Here $(v)$ stands for vertex, $(l)$ for side left, and $(r)$ for side right.) We begin with

$$
\begin{gathered}
S_{(r)}=-32 \pi \alpha \epsilon^{3} \lambda^{3} \int d^{3} x d^{3} y d^{3} z \int \frac{d E_{1}}{2 \pi} \int \frac{d E_{2}}{2 \pi} \int \frac{d^{4} k}{(2 \pi)^{3}} \frac{e^{i \vec{k} \cdot(\vec{x}-\vec{y})}}{k^{2}} \frac{1}{\epsilon^{2}+\left(k_{0}+E_{1}-\epsilon_{v}\right)^{2}} \\
\frac{1}{\epsilon^{2}+\left(E_{1}-E_{2}+k_{0}\right)^{2}} \frac{1}{\epsilon^{2}+\left(E_{2}-\epsilon_{v}\right)^{2}} \bar{\psi}_{v}(\vec{x}) \gamma_{\mu} S_{F}\left(\vec{x}, \vec{y} ; E_{1}\right) \gamma^{\mu} S_{F}\left(\vec{y}, \vec{z}, E_{2}\right) V(z) \psi_{v}(\vec{z}) .
\end{gathered}
$$

In the above, the terms with $\epsilon^{2}$ in the denominator act to emphasize the region of integration in which $E_{1}=\epsilon_{v}-k_{0}$ and $E_{2}=\epsilon_{v}$, and when it is possible to use these values in the Feynman propagators $S_{F}$, the integrations over $E_{1}$ and $E_{2}$ can be immediately carried out. However, if we now make a spectral decomposition of the second Feynman propagator, reexpressing $S_{(r)}$ as

$$
\begin{array}{r}
S_{(r)}=-8 \pi i \epsilon^{3} \lambda^{3} \sum_{m} \int d^{3} z \int \frac{d E_{1}}{2 \pi} \int \frac{d E_{2}}{2 \pi} \frac{1}{\epsilon^{2}+\left(k_{0}+E_{1}-\epsilon_{v}\right)^{2}} \frac{1}{\epsilon^{2}+\left(E_{1}-E_{2}+k_{0}\right)^{2}} \\
\frac{1}{\epsilon^{2}+\left(E_{2}-\epsilon_{v}\right)^{2}} \Sigma_{v m}\left(E_{1}\right) \bar{\psi}_{m}(z) V(z) \psi_{v}(\vec{z}) \frac{1}{E_{2}-\epsilon_{m}(1-i \delta)},
\end{array}
$$


we see that more care is needed when $m=v$. We now concentrate on this case. If we make the approximation $\Sigma_{v m}\left(E_{1}\right)=\Sigma_{v m}\left(\epsilon_{v}-k_{0}\right)$, it is possible to directly carry out the $E_{1}$ and $E_{2}$ integrations, with the result that gives the divergent energy

$$
\Delta E_{(r)}^{\text {div }}=-\frac{3}{2 \epsilon} E^{(1)}(v) \Sigma_{v v}\left(\epsilon_{v}\right)
$$

When taken together with an identical term from the $(l)$ calculation, it is seen that the divergent contributions to the energy cancel, as they must. However, because we have found divergences, terms normally safely dropped can contribute a finite amount to the energy. The only approximation we made in the above was to replace $E_{1}$ with $\epsilon_{v}-k_{0}$ in the self energy. In order to pick up the derivative term, we now expand

$$
\Sigma_{v v}\left(E_{1}\right)=\Sigma_{v v}\left(\epsilon_{v}-k_{0}\right)+\left.\left(E_{1}-\epsilon_{v}+k_{0}\right) \frac{\partial \Sigma_{v v}(E)}{\partial E}\right|_{E=\epsilon_{v}-k_{0}}+\cdots .
$$

The second term can now be easily evaluated, as can an identical term from the (l) case, and we find

$$
\Delta E_{\text {deriv }}^{(l)+(r)}=E^{(1)}(v) \Sigma_{v v}^{\prime}(\omega),
$$

where we have used the fact that the energy depends on $\epsilon_{v}$ through $\omega=\epsilon_{v}-\epsilon_{m}$.

The remaining part of the $(l)$ and $(r)$ terms are easily evaluated, and can be shown to coincide with the results of ordinary perturbation theory. Turning to the vertex term, we first note that while it has no $1 / \epsilon$ divergences, it also contains a derivative term. This can be seen most clearly when a spectral decomposition of both electron propagators in the vertex term is made, in which case we find

$$
\begin{array}{r}
\Delta E_{(v)}=-4 \pi i \alpha \sum_{i j} \int d^{3} z \bar{\psi}_{i}(\vec{z}) V(z) \psi_{j}(\vec{z}) \int \frac{d^{4} k}{(2 \pi)^{4}} \int d^{3} x \int d^{3} y \frac{e^{i \vec{k} \cdot(\vec{x}-\vec{y})}}{k^{2}} \\
\frac{\bar{\psi}_{v}(\vec{x}) \gamma_{\mu} \psi_{i}(\vec{x}) \bar{\psi}_{j}(\vec{y}) \gamma^{\mu} \psi_{v}(\vec{y})}{\left(\epsilon_{v}-k_{0}-\epsilon_{m}(1-i \delta)\right)\left(\epsilon_{v}-k_{0}-\epsilon_{n}(1-i \delta)\right)} .
\end{array}
$$

As with the self-energy, the only source of an imaginary part of $\Delta E_{(v)}$ is pole terms, in which one or other of the states $i$ or $j$ is more deeply bound than the state $v$. The derivative term comes from the term in which both $i$ and $j$ are the state $m$ defined above. But in that case, we see that the integral over $d^{3} z$ in the above equation gives $E^{(1)}(m)$. It is then straightforward to show that, defining $\delta \omega \equiv E^{(1)}(v)-E^{(1)}(m)$, the combined derivative terms taken together with the lowest-order energy gives

$$
\Sigma_{v v}(\omega)+\Delta E_{\text {deriv }}=\left(1+\delta \omega \frac{\partial}{\partial E}\right) \Sigma_{v v}(E)_{E=\omega},
$$

which is clearly the beginning of the Taylor expansion of $\Sigma_{v v}(\omega+\delta \omega)$. In this way, we see that the derivative terms are indeed acting to correct the lowest-order energy difference $\omega$ to its actual value. We emphasize here that the 
standard procedure of using experimental energies in decay rate calculations already accounts for the effect of the derivative term, but the above discussion provides a rigorous theoretical justification for the procedure. In addition to the derivative terms, there are, of course, the standard perturbation theory contributions. They can be accounted for by adding to $R(\omega)$ the quantity

$$
\delta R(\omega)=\sqrt{\frac{J+1}{J}}\left(\sum_{i \neq m} \frac{\left\langle v \| t_{J}^{\lambda}|| i\right\rangle\langle i|U| m\rangle}{\epsilon_{m}-\epsilon_{i}}+\sum_{i \neq v} \frac{\langle v|U| i\rangle\left\langle i|| t_{J}^{\lambda}|| m\right\rangle}{\epsilon_{v}-\epsilon_{i}}\right),
$$

with the understanding that only cross terms in $(R(\omega)+\delta R(\omega))^{2}$ are to be kept.

To illustrate how this calculation works, we consider the calculation of the $M 1$ decay of the $2 s$ state of hydrogenlike thallium as a perturbation of hydrogenlike mercury. In atomic units, the imaginary part of the energy for mercury is $-4.92710^{-4}$, and for thallium $-5.64810^{-4}$, so perturbation theory must pick up $-0.72110^{-4}$ a.u.. The non-derivative parts of the calculation, carried out in Feynman gauge, give $-6.41010^{-4}$ a.u.. The derivative terms contribute 5.736 $10^{-4}$ a.u., for a combined $-0.67410^{-4}$. Note that while the bulk of the difference is picked up in this order of perturbation theory, a smaller difference is left over that is consistent with the expected size of the next order of perturbation theory.

While we have employed a fully field theoretic approach to derive the derivative terms, we now note that if one treats energy-dependent potentials or amplitudes of energy-dependent operators in ordinary Rayleigh-Schrödinger perturbation theory, such terms automatically appear. This is simply because, when we expand $E=E^{(0)}+E^{(1)}+\cdots$, an energy-dependent potential or operator must also be expanded as $T(E)=T\left(E^{(0)}\right)+E^{(1)} T^{\prime}\left(E^{(0)}\right)+\cdots$. Thus, if an amplitude has energy dependence, as is the case for electromagnetic transitions, derivative terms will arise. Returning to our field-theoretic discussion, we note that $\operatorname{Im} \Delta E \propto \omega R^{2}(\omega)$, where $R(\omega)$ is essentially the reduced matrix element of the transition operator $t_{J}^{(\lambda)}(\omega)$. The derivative acting on $\operatorname{Im} \Delta E$ gives a contribution $2 \omega R(\omega) R^{\prime}(\omega)$ that could be obtained from the arguments just given about Rayleigh-Schrödinger perturbation theory. However, the derivative also acts on the linear factor $\omega$, and can clearly be accounted for by replacing $\omega$ with $\omega+\delta \omega$ in the expression for $\operatorname{Im} \Delta E$. We have been unable to find an alternative derivation for this effect, and therefore rely on the more elaborate field theory approach given above to explain it.

In carrying out perturbation theory for the decays of helium in the next section, we adopt the following procedure. In lowest order, we will use the above equation with the matrix elements $R(\omega)$ suitably generalized to helium. We will show that in second-order perturbation theory the derivative terms are not only present, but play a crucial role in preserving gauge invariance. Finally, although we have not carried out a complete field theoretic analysis for helium (which involves treating the imaginary part of all two-photon diagrams), we will change the outside factor $\omega$. However, instead of including only first-order perturbation theory, as done in this section, we also include all the higher-order 
perturbation theory, recoil, and QED effects calculated in Refs. [3, 4], which amounts to using the experimental energies.

\section{Application of Perturbation Theory to Heli- umlike Ions}

In this section, we carry out a gauge-invariant second-order perturbation theory calculation of amplitudes for transitions in heliumlike ions fashioned after the QED calculation of Sec. 3.

\subsection{Basic equations}

We consider single-configuration states such as $2{ }^{3} P_{0}$ or $1{ }^{1} S_{0}$ and carry out our analysis in the uncoupled representation. Let us consider two unperturbed wave functions, $\Psi_{v a}^{(0)}$ and $\Psi_{w b}^{(0)}$,

$$
\begin{aligned}
& \Psi_{v a}^{(0)}=a_{v}^{\dagger} a_{a}^{\dagger}|0\rangle \\
& \Psi_{w b}^{(0)}=a_{w}^{\dagger} a_{b}^{\dagger}|0\rangle .
\end{aligned}
$$

Here, the orbital labels $a$ and $b$ refer to $1 s$ electrons and the labels $v$ and $w$ refer to excited electrons. We allow for the possibility that $v$ is also a $1 s$ state, in which case, the wave function $\Psi_{v a}^{(0)}$ represents the $(1 s)^{2}$ ground state. Later, these states will be coupled to give a good value of angular momentum. The energies of the states are $E_{v a}^{(0)}=\epsilon_{v}+\epsilon_{a}$ and $E_{w b}^{(0)}=\epsilon_{w}+\epsilon_{b}$. The energy of the absorbed photon is then, in lowest order, $\hbar \omega=E_{w b}^{(0)}-E_{v a}^{(0)}=\epsilon_{w}-\epsilon_{v}$.

The lowest-order amplitude for the transition induced by the interaction Hamiltonian $H_{I}$ of Eq.(31) is

$$
T^{(1)}=\left\langle\Psi_{w b}^{(0)}\left|H_{I}\right| \Psi_{v a}^{(0)}\right\rangle=\left(h_{I}\right)_{w v} \delta_{b a}-\left(h_{I}\right)_{w a} \delta_{b v}
$$

The amplitude $T^{(1)}$ is obviously gauge invariant, since according to Eq.(29), the two single-particle matrix elements are themselves independent of gauge for $\hbar \omega=\epsilon_{w}-\epsilon_{v}$.

We write the first-order correction to $\Psi_{v a}^{(0)}$ as

$$
\Psi_{v a}^{(1)}=\sum_{i j \neq v a} \rho_{i j v a}^{(1)} a_{i}^{\dagger} a_{j}^{\dagger}|0\rangle .
$$

This perturbed wave function satisfies

$$
\left(H_{0}-E_{v a}^{(0)}\right) \Psi_{v a}^{(1)}=\left(E_{v a}^{(1)}-V_{I}\right) \Psi_{v a}^{(0)} .
$$

We use the local potential $U(r)$ given in Eq.(4) to approximate the electronelectron interaction in the single-particle Dirac equation. This is expected to 
improve the quality of the approximate wave functions as well as the convergence of the perturbation expansion. We find for the first-order energy

$$
E_{v a}^{(1)}=\tilde{v}_{v a v a}-U_{v v}-U_{a a},
$$

where $\tilde{v}_{i j k l}$ is defined in Eq.(11). The first-order correction to the photon energy is

$$
\delta \omega=\frac{E_{w b}^{(1)}-E_{v a}^{(1)}}{\hbar} .
$$

For $i j \neq v a$, we find that the expansion coefficients in the first-order wave function are

$$
\rho_{i j v a}^{(1)}=-\frac{v_{i j v a}}{\epsilon_{i}+\epsilon_{j}-\epsilon_{v}-\epsilon_{a}}+\frac{U_{i v} \delta_{j a}}{\epsilon_{i}-\epsilon_{v}}+\frac{U_{j a} \delta_{i v}}{\epsilon_{j}-\epsilon_{a}}
$$

The corresponding second-order transition amplitude is given by

$$
\begin{aligned}
T^{(2)} & =\left\langle\Psi_{w b}^{(1)}\left|H_{I}\right| \Psi_{v a}^{(0)}\right\rangle+\left\langle\Psi_{w b}^{(0)}\left|H_{I}\right| \Psi_{v a}^{(1)}\right\rangle+\delta \omega \frac{d T^{(1)}}{d \omega} \\
& =\left\langle\Psi_{w b}^{(1)}\left|H_{I}\right| \Psi_{v a}^{(0)}\right\rangle+\left\langle\Psi_{w b}^{(0)}\left|H_{I}\right| \Psi_{v a}^{(1)}\right\rangle+\delta \omega\left\langle\Psi_{w b}^{(0)}\left|\frac{d H_{I}}{d \omega}\right| \Psi_{v a}^{(0)}\right\rangle,
\end{aligned}
$$

where the third term on the right-hand side is the derivative term discussed in Sec. 3. With the first-order wave function (75), we obtain

$$
\begin{aligned}
T^{(2)} & =\sum_{i j \neq w b} \rho_{i j w b}^{(1) *}\left\langle 0\left|a_{j} a_{i} H_{I} a_{v}^{\dagger} a_{a}^{\dagger}\right| 0\right\rangle+\sum_{i j \neq v a} \rho_{i j v a}^{(1)}\left\langle 0\left|a_{b} a_{w} H_{I} a_{i}^{\dagger} a_{j}^{\dagger}\right| 0\right\rangle \\
& +\delta \omega\left\langle 0\left|a_{b} a_{w} \frac{d H_{I}}{d \omega} a_{v}^{\dagger} a_{a}^{\dagger}\right| 0\right\rangle .
\end{aligned}
$$


This expression can, in turn, be rewritten as

$$
\begin{aligned}
T^{(2)} & =\sum_{i j \neq w b} \rho_{i j w b}^{(1) *}\left[\left(h_{I}\right)_{i v} \delta_{j a}-\left(h_{I}\right)_{i a} \delta_{j v}+\left(h_{I}\right)_{j a} \delta_{i v}-\left(h_{I}\right)_{j v} \delta_{i a}\right] \\
& +\sum_{i j \neq v a} \rho_{i j v a}^{(1)}\left[\left(h_{I}\right)_{w i} \delta_{b j}-\left(h_{I}\right)_{b i} \delta_{w j}+\left(h_{I}\right)_{b j} \delta_{w i}-\left(h_{I}\right)_{w j} \delta_{b i}\right] \\
& +\delta \omega\left[\left(\frac{d h_{I}}{d \omega}\right)_{w v} \delta_{b a}-\left(\frac{d h_{I}}{d \omega}\right)_{w a} \delta_{b v}\right] \\
& =\sum_{i a \neq w b} \frac{v_{w b i a}\left(h_{I}\right)_{i v}-v_{w b a i}\left(h_{I}\right)_{i v}}{\epsilon_{w}-\epsilon_{i}}-\sum_{i v \neq w b} \frac{v_{w b v i}\left(h_{I}\right)_{i a}-v_{w b i v}\left(h_{I}\right)_{i a}}{\epsilon_{i}+\epsilon_{v}-\epsilon_{w}-\epsilon_{b}} \\
& +\sum_{i b \neq v a} \frac{\left(h_{I}\right)_{w i} v_{i b v a}-\left(h_{I}\right)_{w i} v_{b i v a}}{\epsilon_{v}-\epsilon_{i}}-\sum_{i} \frac{\left(h_{I}\right)_{b i} v_{w i v a}-\left(h_{I}\right)_{b i} v_{i w v a}}{\epsilon_{i}+\epsilon_{w}-\epsilon_{v}-\epsilon_{a}} \\
& +\sum_{i \neq w} \frac{U_{w i}\left(h_{I}\right)_{i v} \delta_{b a}-U_{w i}\left(h_{I}\right)_{i a} \delta_{b v}}{\epsilon_{i}-\epsilon_{w}} \\
& +\sum_{i \neq v} \frac{\left(h_{I}\right)_{w i} U_{i v}}{\epsilon_{i}-\epsilon_{v}} \delta_{b a}-\sum_{i \neq a} \frac{\left(h_{I}\right)_{w i} U_{i a}}{\epsilon_{i}-\epsilon_{a}} \delta_{b v} \\
& +\delta_{\omega}\left[\left(\frac{d h_{I}}{d \omega}\right)_{w v} \delta_{b a}-\left(\frac{d h_{I}}{d \omega}\right)_{w a} \delta_{b v}\right]
\end{aligned}
$$

Equation (82) is the basic equation for the second-order transition amplitude. In contrast to the no-pair approach, we sum over both positive- and negativeenergy states as we would if we were evaluating the transition amplitude from the QED point of view.

We are now in a position to prove the gauge invariance of $T^{(2)}$. For this purpose, we replace $h_{I}$ by $h_{I}+\Delta h_{I}$ everywhere in the expression above and examine the resulting change $\Delta T^{(2)}$. With the aid of Eq.(29), we obtain

$$
\begin{aligned}
\Delta T^{(2)}= & -\frac{i e}{\hbar}\left\{-\sum_{i a \neq w b} \tilde{v}_{w b i a}(\chi)_{i v}-\sum_{i v \neq w b} \tilde{v}_{w b v i}(\chi)_{i a}\right. \\
+ & \sum_{i b \neq v a}(\chi)_{w i} \tilde{v}_{i b v a}+\sum_{i}(\chi)_{b i} \tilde{v}_{w i v a} \\
+ & \sum_{i \neq w}\left[U_{w i}(\chi)_{i v} \delta_{b a}-U_{w i}(\chi)_{i a} \delta_{b v}\right] \\
- & \sum_{i \neq v}(\chi)_{w i} U_{i v} \delta_{b a}+\sum_{i \neq a}(\chi)_{w i} U_{i a} \delta_{b v} \\
& \left.-\left(E_{w b}^{(1)}-E_{v a}^{(1)}\right)\left[(\chi)_{w v} \delta_{b a}-(\chi)_{w a} \delta_{b v}\right]\right\}
\end{aligned}
$$


This expression can be rewritten as:

$$
\begin{aligned}
\Delta T^{(2)}= & -\frac{i e}{\hbar}\left\{\sum_{i}\left[-\tilde{v}_{w b i a}(\chi)_{i v}-\tilde{v}_{w b v i}(\chi)_{i a}+(\chi)_{w i} \tilde{v}_{i b v a}+(\chi)_{b i} \tilde{v}_{w i v a}\right]\right. \\
& +\sum_{i}\left[\left(U_{w i}(\chi)_{i v}-(\chi)_{w i} U_{i v}\right) \delta_{b a}-\left(U_{w i}(\chi)_{i a}-(\chi)_{w i} U_{i a}\right) \delta_{b v}\right] \\
& +\left[\tilde{v}_{w b w b}-U_{w w}-U_{b b}-\tilde{v}_{v a v a}+U_{v v}+U_{a a}\right](\chi)_{w v} \delta_{b a} \\
& -\left[\tilde{v}_{w b w b}-U_{w w}-U_{b b}-\tilde{v}_{v a v a}+U_{v v}+U_{a a}\right](\chi)_{w a} \delta_{b v} \\
& \left.-\left(E_{w b}^{(1)}-E_{v a}^{(1)}\right)\left[(\chi)_{w v} \delta_{b a}-(\chi)_{w a} \delta_{b v}\right]\right\} .
\end{aligned}
$$

The sums on the first two lines vanish identically if completeness of the intermediate states $i$ is invoked. This fact is demonstrated in the Appendix. Furthermore, from Eq.(77), the terms on the third and fourth lines are seen to cancel the term on the last line. We therefore find that

$$
\Delta T^{(2)}=0,
$$

provided one sums over both positive- and negative-energy intermediate states.

If we were carrying out an MBPT calculation of the amplitude using the no-pair interaction, the negative-energy states in (82) would not appear and we would lose the gauge invariance. We expect the dominant contributions to the all-order no-pair amplitudes from negative-energy states to be accurately predicted by the negative-energy contributions to Eq.(82). Moreover, if the negative-energy contributions from perturbation theory are added to the exact no-pair amplitudes in length gauge and in velocity gauge, then, we expect the "corrected" no-pair amplitudes in these two gauges to agree with one another to a high degree of accuracy. We verify the correctness of these conjectures in Sec. 4.3.

\subsection{Angular reduction}

To compare the analysis given in the previous subsection with that for the nopair amplitudes given in Sec. 2, we must first replace the interaction Hamiltonian $h_{I}$ used in the equations of the previous subsection with the transition operator $t_{J}^{(\lambda)}$ defined in Sec. 2.3. We couple the orbitals $v$ and $a$ in the initial-state wave function to angular momentum $J_{I}, M_{I}$,

$$
\left|J_{I} M_{I}\right\rangle=\eta_{v a} \sum_{m_{v} m_{a}}\left\langle j_{v} m_{v} j_{a} m_{a} \mid J_{I} M_{I}\right\rangle \Psi_{v a}
$$

where $\eta_{v a}=1 / \sqrt{2}$ for the identical particle case $\left(n_{a}=n_{v}\right.$ and $\left.\kappa_{a}=\kappa_{v}\right)$ and $\eta_{v a}=1$, otherwise. Similarly, we couple the final-state orbitals $w$ and $b$ to angular momentum $J_{F}, M_{F}$,

$$
\left|J_{F} M_{F}\right\rangle=\sum_{m_{w} m_{b}}\left\langle j_{w} m_{w} j_{b} m_{b} \mid J_{F} M_{F}\right\rangle \Psi_{w b}
$$


Carrying out the sums over magnetic quantum numbers, we obtain the following expression for the reduced matrix element of the first-order transition amplitude,

$$
\begin{aligned}
\left\langle F\left\|T_{J}^{(\lambda)}\right\| I\right\rangle^{(1)}= & \eta_{v a} \sqrt{\left[J_{F}\right]\left[J_{I}\right]}\left\{(-1)^{J_{I}+J+w+a}\left\{\begin{array}{ccc}
J_{F} & J_{I} & J \\
v & w & b
\end{array}\right\}\left\langle w\left\|t_{J}^{(\lambda)}\right\| v\right\rangle \delta_{b a}\right. \\
& \left.+(-1)^{J+w+a}\left\{\begin{array}{ccc}
J_{F} & J_{I} & J \\
a & w & b
\end{array}\right\}\left\langle w\left\|t_{J}^{(\lambda)}\right\| a\right\rangle \delta_{b v}\right\} \cdot
\end{aligned}
$$


Similarly, the reduced-matrix element of the transition amplitude in secondorder perturbation theory is found to be

$$
\begin{aligned}
& \left\langle F|| T_{J}^{(\lambda)} \| I\right\rangle^{(2)}=\eta_{v a} \sqrt{\left[J_{F}\right]\left[J_{I}\right]} \times \\
& \left\{\sum_{i a \neq w b, L}(-1)^{J_{I}+J+J_{F}+L}\left\{\begin{array}{ccc}
w & b & J_{F} \\
a & i & L
\end{array}\right\}\left\{\begin{array}{ccc}
J_{F} & J_{I} & J \\
v & i & a
\end{array}\right\} \frac{X_{L}(w b i a)\left\langle i\left\|t_{J}^{(\lambda)}\right\| v\right\rangle}{\epsilon_{w}-\epsilon_{i}}\right. \\
& +\sum_{i a \neq w b, L}(-1)^{J_{I}+J+L}\left\{\begin{array}{ccc}
w & b & J_{F} \\
i & a & L
\end{array}\right\}\left\{\begin{array}{ccc}
J_{F} & J_{I} & J \\
v & i & a
\end{array}\right\} \frac{X_{L}(w b a i)\left\langle i\left\|t_{J}^{(\lambda)}\right\| v\right\rangle}{\epsilon_{w}-\epsilon_{i}} \\
& +\sum_{i v \neq w b, L}(-1)^{J+L+b+a}\left\{\begin{array}{ccc}
w & b & J_{F} \\
i & v & L
\end{array}\right\}\left\{\begin{array}{ccc}
J_{F} & J_{I} & J \\
a & i & v
\end{array}\right\} \frac{X_{L}(w b v i)\left\langle i\left\|t_{J}^{(\lambda)}\right\| a\right\rangle}{\epsilon_{i}+\epsilon_{v}-\epsilon_{w}-\epsilon_{b}} \\
& +\sum_{i v \neq w b, L}(-1)^{J_{F}+J+L+b+a}\left\{\begin{array}{ccc}
w & b & J_{F} \\
v & i & L
\end{array}\right\}\left\{\begin{array}{ccc}
J_{F} & J_{I} & J \\
a & i & v
\end{array}\right\} \frac{X_{L}(w b i v)\left\langle i\left\|t_{J}^{(\lambda)}\right\| a\right\rangle}{\epsilon_{i}+\epsilon_{v}-\epsilon_{w}-\epsilon_{b}} \\
& +\sum_{i b \neq v a, L}(-1)^{J+L+w-v}\left\{\begin{array}{ccc}
v & a & J_{I} \\
b & i & L
\end{array}\right\}\left\{\begin{array}{ccc}
J_{F} & J_{I} & J \\
i & w & b
\end{array}\right\} \frac{\left\langle w\left\|t_{J}^{(\lambda)}\right\| i\right\rangle X_{L}(i b v a)}{\epsilon_{v}-\epsilon_{i}} \\
& +\sum_{i b \neq v a, L}(-1)^{J_{I}+J+L+w-v}\left\{\begin{array}{ccc}
v & a & J_{I} \\
i & b & L
\end{array}\right\}\left\{\begin{array}{ccc}
J_{F} & J_{I} & J \\
i & w & b
\end{array}\right\} \frac{\left\langle w\left\|t_{J}^{(\lambda)}\right\| i\right\rangle X_{L}(\text { biva })}{\epsilon_{v}-\epsilon_{i}} \\
& +\sum_{i, L}(-1)^{J_{I}+J+J_{F}+L+w+v}\left\{\begin{array}{ccc}
v & a & J_{I} \\
i & w & L
\end{array}\right\}\left\{\begin{array}{ccc}
J_{F} & J_{I} & J \\
i & b & w
\end{array}\right\} \frac{\left\langle b\left\|t_{J}^{(\lambda)}\right\| i\right\rangle X_{L}(w i v a)}{\epsilon_{i}+\epsilon_{w}-\epsilon_{v}-\epsilon_{a}} \\
& +\sum_{i, L}(-1)^{J+J_{F}+L+w+v}\left\{\begin{array}{ccc}
v & a & J_{I} \\
w & i & L
\end{array}\right\}\left\{\begin{array}{ccc}
J_{F} & J_{I} & J \\
i & b & w
\end{array}\right\} \frac{\left\langle b\left\|t_{J}^{(\lambda)}\right\| i\right\rangle X_{L}(i w v a)}{\epsilon_{i}+\epsilon_{w}-\epsilon_{v}-\epsilon_{a}} \\
& +(-1)^{J_{I}+J+w+a}\left\{\begin{array}{ccc}
J_{F} & J_{I} & J \\
v & w & a
\end{array}\right\} \delta_{b a} \sum_{i \neq w} \delta_{\kappa_{i} \kappa_{w}} \frac{U_{w i}\left\langle i\left\|t_{J}^{(\lambda)}\right\| v\right\rangle}{\epsilon_{i}-\epsilon_{w}} \\
& +(-1)^{J+w+a}\left\{\begin{array}{ccc}
J_{F} & J_{I} & J \\
a & w & v
\end{array}\right\} \delta_{b v} \sum_{i \neq w} \delta_{\kappa_{i} \kappa_{w}} \frac{U_{w i}\left\langle i\left\|t_{J}^{(\lambda)}\right\| a\right\rangle}{\epsilon_{i}-\epsilon_{w}} \\
& +(-1)^{J_{I}+J+w+a}\left\{\begin{array}{ccc}
J_{F} & J_{I} & J \\
v & w & a
\end{array}\right\} \delta_{b a} \sum_{i \neq v} \delta_{\kappa_{i} \kappa_{v}} \frac{\left\langle w\left\|t_{J}^{(\lambda)}\right\| i\right\rangle U_{i v}}{\epsilon_{i}-\epsilon_{v}} \\
& +(-1)^{J+w+a}\left\{\begin{array}{ccc}
J_{F} & J_{I} & J \\
a & w & v
\end{array}\right\} \delta_{b v} \sum_{i \neq a} \delta_{\kappa_{i} \kappa_{a}} \frac{\left\langle w\left\|t_{J}^{(\lambda)}\right\| i\right\rangle U_{i a}}{\epsilon_{i}-\epsilon_{a}} \\
& +\frac{E_{w b}^{(1)}-E_{v a}^{(1)}}{\hbar}\left[(-1)^{J_{I}+J+w+a}\left\{\begin{array}{ccc}
J_{F} & J_{I} & J \\
v & w & a
\end{array}\right\}\left\langle w\left\|\left(\frac{d t_{J}^{(\lambda)}}{d \omega}\right)\right\| v\right\rangle \delta_{b a}\right. \\
& \left.\left.+(-1)^{J+w+a}\left\{\begin{array}{ccc}
J_{F} & J_{I} & J \\
a & w & v
\end{array}\right\}\left\langle w\left\|\left(\frac{d t_{J}^{(\lambda)}}{d \omega}\right)\right\| a\right\rangle \delta_{b v}\right]\right\} \text {. }
\end{aligned}
$$


With the notation

$$
\left\langle F\left\|T_{J}^{(\lambda)}\right\| I\right\rangle^{(1+2)}=\left\langle F\left\|T_{J}^{(\lambda)}\right\| I\right\rangle^{(1)}+\left\langle F\left\|T_{J}^{(\lambda)}\right\| I\right\rangle^{(2)},
$$

the Einstein A-coefficients are given by

$$
A_{J}^{(\lambda)}=2 \alpha(\omega+\delta \omega) \frac{[J]}{\left[J_{I}\right]} \frac{J+1}{J}\left|\left\langle F\left\|T_{J}^{(\lambda)}\right\| I\right\rangle^{(1+2)}\right|^{2} .
$$

For comparison with no-pair theory, we need expressions for the reduced matrix elements of the multipole-moment operators. These are related to the reduced matrix elements of the transition operators by the expressions

$$
\left\langle F\left\|T_{J}^{(\lambda)}\right\| I\right\rangle^{(1+2)}=\frac{\alpha^{k}(\omega+\delta \omega)^{k}}{(2 J+1) ! !} \begin{cases}\left\langle F\left\|Q_{J}^{(1)}\right\| I\right\rangle^{(1+2)} & \text { for } \lambda=1 \\ (\alpha / 2)\left\langle F\left\|M_{J}\right\| I\right\rangle^{(1+2)} & \text { for } \lambda=0\end{cases}
$$

As a specific example, let us consider the $2{ }^{3} P_{0} \rightarrow 2{ }^{3} S_{1}$ transition. We use the Eqs.(87) and (88) to evaluate the transition amplitudes, and Eq.(90) to obtain the reduced matrix element of the electric-dipole moment operator. In Table 3, we list the contributions to length-form and velocity-form electric-dipole moment matrix elements for $Z=20,40$, and 80 . We see that the first-order matrix elements are identical in length and velocity forms. The sum over intermediate states in (88) is seen to contribute very little to the length-form moments, but substantially to the velocity-form matrix elements. By contrast, the derivative terms in Eq.(88) are seen to contribute substantially to the length form, but give only tiny contributions to the velocity form. When the contributions from both parts of the second-order calculation are added, we obtain gauge-invariant second-order matrix elements. Summing the first-order and second-order contributions gives values that are not only gauge invariant, but are also in good quantitative agreement with the length-form no-pair results presented, for example, in Table 2 .

\subsection{Negative-energy components of second-order ampli- tudes}

In this subsection, we examine the decomposition of the transition amplitudes calculated in second-order perturbation theory into positive-energy and negativeenergy components. The negative-energy contributions to the transition amplitude arise from those terms in the sum over states $i$ in the second-order amplitude given in Eq.(88) for which $\epsilon_{i}<-2 m c^{2}$.

The value of the sum over $i$ and the corresponding contributions from negativeenergy states depend on the potential used in defining the lowest-order Hamiltonian. Thus, the negative-energy contributions to the amplitudes obtained in a calculation starting from $U(r)=0$ (the Coulomb-field case) will be quite different from the contributions in a calculation starting from $U(r)=v_{0}(1 s, r)$, (the Hartree case). The reason for this difference is obvious on inspecting Eq.(88). In the Hartree case, there are substantial cancellations between the 
Table 3: Perturbation theory applied to the reduced matrix element of the dipole-moment operator in length-form and in velocity-form for the $2{ }^{3} P_{0} \rightarrow$ $2{ }^{3} S_{1}$ transition. The contribution "1st" is from first-order perturbation theory. The contributions " $\Sigma_{i}$ " and " $d T^{(1)} / d \omega$ are from the sum over states and the derivative term in second-order perturbation theory. The rows labeled "2nd" and "1st+2nd" are the second-order contributions and the sum of the firstand second-order contributions, respectively. Results are calculated for the case where $U(r)=v_{0}(1 s, r)$ and do not include the Breit interactions .

\begin{tabular}{llrr}
\hline \hline$Z$ & Contribution & $\left\langle F|| Q_{1} \| I\right\rangle_{l}$ & $\left\langle F\left\|Q_{1}\right\| I\right\rangle_{v}$ \\
\hline 20 & 1st & 0.132096 & 0.132096 \\
& $\Sigma_{i}$ & -0.001058 & 0.022721 \\
& $d T^{(1)} / d \omega$ & 0.023780 & 0.000001 \\
& 2nd & 0.022722 & 0.022722 \\
& 1st+2nd & 0.154818 & 0.154818 \\
& & & \\
40 & 1st & 0.061919 & 0.061919 \\
& $\Sigma_{i}$ & -0.000246 & 0.011911 \\
& $d T^{(1)} / d \omega$ & 0.012157 & 0.000001 \\
& 2nd & 0.011911 & 0.011911 \\
& 1st+2nd & 0.073830 & 0.073830 \\
& & & \\
80 & 1st & 0.024229 & 0.024229 \\
& $\Sigma_{i}$ & -0.000049 & 0.007958 \\
& $d T^{(1)} / d \omega$ & 0.008008 & 0.000001 \\
& 2nd & 0.007959 & 0.007959 \\
& 1st+2nd & 0.032188 & 0.032188 \\
\hline \hline
\end{tabular}


Table 4: Comparison of length-form $\left\langle F|| Q_{1}|| I\right\rangle_{l}$ and velocity-form $\left\langle F|| Q_{1} \| I\right\rangle_{v}$ dipole matrix elements from no-pair, "no-pair", calculations and from secondorder perturbation theory, "pert-th". Here, superscripts (+) and (-) refer to contributions from positive- and negative-energy states, respectively. $\Delta Q^{(+)}$is the difference in the positive-energy parts of the length-form and velocity-form amplitudes. All calculations are carried out in a Coulomb basis, $U(r)=0$. The notation $a(-b)$ designates $a \times 10^{-b}$.

\begin{tabular}{|c|c|c|c|c|c|c|}
\hline$Z$ & Type & $\left\langle\left\langle\overline{\left\langle F|| Q_{1} \| I\right\rangle_{l}^{(+)}}\right.\right.$ & $\left\langle\left\langle\overline{\left\langle F|| Q_{1}|| I\right\rangle_{l}^{(-)}}\right.\right.$ & $\left\langle\left\langle\overline{\left\langle F\left\|Q_{1}\right\| I\right\rangle_{v}^{(+)}}\right.\right.$ & $\left\langle\left\langle\overline{\left\langle F|| Q_{1}|| I\right\rangle_{v}^{(-)}}\right.\right.$ & $\Delta Q^{(+)}$ \\
\hline \multicolumn{7}{|c|}{$2{ }^{3} P_{0}-2{ }^{3} S_{1}$} \\
\hline 20 & no-pair & 0.154750 & & 0.154400 & & 0.000350 \\
\hline 20 & pert-th & 0.148663 & $1(-12)$ & 0.148281 & 0.000382 & 0.000382 \\
\hline 50 & no-pair & 0.057636 & & 0.056543 & & 0.001093 \\
\hline 50 & pert-th & 0.056582 & $5(-10)$ & 0.055457 & 0.001125 & 0.001125 \\
\hline 100 & no-pair & 0.022809 & & 0.020442 & & 0.002367 \\
\hline 100 & pert-th & 0.022378 & $2(-07)$ & 0.020006 & 0.002374 & 0.002372 \\
\hline \multicolumn{7}{|c|}{$2^{3} P_{2}-2^{3} S_{1}$} \\
\hline 20 & no-pair & 0.347546 & & 0.347046 & & 0.000500 \\
\hline 20 & pert-th & 0.337611 & $4(-09)$ & 0.337055 & 0.000557 & 0.000556 \\
\hline 50 & no-pair & 0.133056 & & 0.132865 & & 0.000192 \\
\hline 50 & pert-th & 0.132769 & $6(-08)$ & 0.132569 & 0.000203 & 0.000200 \\
\hline 100 & no-pair & 0.059372 & & 0.059365 & & 0.000007 \\
\hline 100 & pert-th & 0.059368 & $2(-07)$ & 0.059360 & $-9(-08)$ & 0.000008 \\
\hline
\end{tabular}

sums on the first eight lines and the counter-terms with $U_{i k}$ on the following four lines; such cancellations cannot occur in the Coulomb case. Since we wish to use the negative-energy contributions from perturbation theory to estimate the negative-energy terms omitted in the no-pair calculations, it is important to carry out both the perturbation-theory calculation and the no-pair calculation starting from the same potential. The energies and length-form matrix elements obtained from the no-pair Hamiltonian are found to be independent of $U(r)$ to better than six significant figures. However, the velocity-form matrix-elements obtained from a no-pair calculation starting from the Hartree potential are quite different from those obtained from a corresponding calculation starting from a Coulomb potential.

These points are illustrated in Tables 4 and 5, where no-pair and perturbation theory amplitudes for the sensitive $2{ }^{3} P_{0} \rightarrow 2{ }^{3} S_{1}$ and $2{ }^{3} P_{2} \rightarrow 2{ }^{3} S_{1}$ transitions at $Z=20,50$, and 100 are decomposed into positive- and negative-energy components and compared. Table 4 gives the amplitudes obtained starting from a Coulomb potential and Table 5 gives those obtained starting from a Hartree potential. The positive-energy part of the length-form and velocity-form am- 
Table 5: Comparison of length-form $\left\langle F|| Q_{1}|| I\right\rangle_{l}$ and velocity-form $\left\langle F|| Q_{1}|| I\right\rangle_{v}$ dipole matrix elements from no-pair, "no-pair", calculations and from secondorder perturbation theory, "pert-th". Here, the superscripts $(+)$ and $(-)$ refer to contributions from positive- and negative-energy states, respectively. $\Delta Q^{(+)}$is the difference in the positive-energy parts of the length-form and velocity-form amplitudes. All calculations are carried out in a Hartree basis, $U(r)=v_{0}(1 s, r)$. The notation $a(-b)$ designates $a \times 10^{-b}$.

\begin{tabular}{|c|c|c|c|c|c|c|}
\hline$Z$ & Type & $\left\langle\left\langle\overline{\left\langle F\left\|Q_{1}\right\| I\right\rangle_{l}^{(+)}}\right.\right.$ & $\left\langle\left\langle\overline{\left\langle F|| Q_{1} \| I\right\rangle_{l}^{(-)}}\right.\right.$ & $\left\langle\overline{\left\langle F\left\|Q_{1}\right\| I\right\rangle_{v}^{(+)}}\right.$ & $\left\langle\left\langle\overline{\left\langle F\left\|Q_{1}\right\| I\right\rangle_{v}^{(-)}}\right.\right.$ & $\Delta Q^{(+)}$ \\
\hline \multicolumn{7}{|c|}{$2^{3} P_{0}-2^{3} S_{1}$} \\
\hline 20 & no-pair & 0.154750 & & 0.154831 & & -0.000081 \\
\hline 20 & pert-th & 0.154936 & $-8(-09)$ & 0.155018 & -0.000082 & -0.000082 \\
\hline 50 & no-pair & 0.057636 & & 0.057345 & & 0.000291 \\
\hline 50 & pert-th & 0.057668 & $-7(-08)$ & 0.057373 & 0.000295 & 0.000295 \\
\hline 100 & no-pair & 0.022809 & & 0.021401 & & 0.001408 \\
\hline 100 & pert-th & 0.022772 & $-2(-07)$ & 0.021349 & 0.001421 & 0.001423 \\
\hline \multicolumn{7}{|c|}{$2^{3} P_{2}-2^{3} S_{1}$} \\
\hline 20 & no-pair & 0.347546 & & 0.346656 & & 0.000890 \\
\hline 20 & pert-th & 0.347808 & $2(-08)$ & 0.346932 & 0.000877 & 0.000876 \\
\hline 50 & no-pair & 0.133056 & & 0.132655 & & 0.000401 \\
\hline 50 & pert-th & 0.133068 & $1(-07)$ & 0.132670 & 0.000399 & 0.000398 \\
\hline 100 & no-pair & 0.059372 & & 0.059311 & & 0.000061 \\
\hline 100 & pert-th & 0.059378 & $4(-07)$ & 0.059321 & 0.000052 & 0.000057 \\
\hline
\end{tabular}


plitudes from perturbation theory are seen to differ from the corresponding no-pair amplitudes by less than $4 \%$ in the Coulomb case and less than $0.2 \%$ in the Hartree case. For both cases, it is seen that there are no contributions to the length-form amplitudes at the level of accuracy of interest here (five significant figures). There are, however, non-negligible negative-energy contributions to the velocity-form amplitudes in perturbation theory. If we use the negative-energy contributions to the perturbation theory velocity-form amplitudes to estimate the missing negative-energy corrections to the velocity-form no-pair amplitudes, then the corrected velocity-form no-pair amplitudes go into agreement with the length-form no-pair amplitudes to four significant figures in the Coulomb-field case and to five significant figures in the Hartree case. Since for either starting potential, the length-form no-pair potential is insensitive to either negative-energy corrections or to the starting potential, this form for the amplitude will be adopted for our final tabulations.

For magnetic-multipole transitions, we have only one form (velocity-form) of the transition operator to consider. Again, we carry out no-pair calculations and perturbation-theory calculations in two potentials, the nuclear Coulomb potential and the Hartree potential. The no-pair amplitudes are found to differ somewhat in the two potentials, because the missing negative-energy contributions to the amplitudes depend on the starting potential. It is found that the positive-energy amplitudes from perturbation theory agree with the no-pair amplitudes at the $1 \%$ level of accuracy for the Coulomb case and at the $0.1 \%$ level for the Hartree case. We use the negative-frequency contributions from perturbation theory to estimate the missing negative-energy contributions from the no-pair amplitudes. The results are shown in Table 6, where we list the nopair amplitudes, the negative-energy contributions from perturbation theory, and the resulting modified amplitudes for M1 transitions $2{ }^{3} S_{1} \rightarrow 1{ }^{1} S_{0}$ and M2 transitions $2{ }^{3} P_{2} \rightarrow 1{ }^{1} S_{0}$, calculated in two potentials. The resulting modified amplitudes are seen to be independent of the starting potential to better than five figures for all M2 transitions and for those M1 transitions with $Z \geq 30$. For $Z<30$, we expect the modified no-pair amplitudes calculated using the Hartree potential to be more accurate than those using the Coulomb potential. We estimate the error in the low $Z$ M1 amplitudes to be less than $0.1 \%$ for $Z<30$.

In summary, we adopt length-form no-pair amplitudes in our final tabulation of E1 transitions, and we adopt no-pair amplitudes modified by adding negative-energy contributions from perturbation theory for magnetic-multipole transitions. For M1 transitions with $Z<30$, our amplitudes are calculated starting from the Hartree potential. In the following section, we present our final results and compare them with other calculations and with experiment.

\section{Results and Comparisons}

In our final tabulations, we use length-form E1 transition amplitudes calculated with exact no-pair wave functions, including both the Coulomb and Breit 
Table 6: Reduced matrix elements of the magnetic dipole moment for $2^{3} S_{1} \rightarrow$ $1^{1} S_{0}$ transitions and the magnetic quadrupole moment for $2^{3} P_{2} \rightarrow 1^{1} S_{0}$ transitions. Values in columns labeled "Hartree" are evaluated using basis functions created in the Hartree potential $U(r)=v_{0}(1 s, r)$, and those listed in columns "Coulomb" are calculated in a Coulomb-field basis. The no-pair moments are modified by adding negative-energy corrections from perturbation theory to give the corrected matrix elements listed in the columns labeled $\langle F|| M \| I\rangle$. The notation $a(-b)$ designates $a \times 10^{-b}$.

\begin{tabular}{ccccccc}
\hline \hline & Hartree & Hartree & Hartree & Coulomb & Coulomb & Coulomb \\
$Z$ & no-pair (+) & pert-th $(-)$ & $\langle F\|M\| I\rangle$ & no-pair (+) & pert-th $(-)$ & $\langle F\|M\| I\rangle$ \\
\hline & & & \multicolumn{2}{c}{$2^{3} S_{1} \rightarrow 1^{1} S_{0}$} \\
5 & $4.26756(-4)$ & $-9.99661(-7)$ & $4.25756(-4)$ & $4.26020(-4)$ & $-2.32841(-8)$ & $4.25997(-4)$ \\
10 & $1.76852(-3)$ & $-1.44150(-6)$ & $1.76708(-3)$ & $1.76749(-3)$ & $-1.62401(-7)$ & $1.76733(-3)$ \\
20 & $7.21056(-3)$ & $-8.04420(-7)$ & $7.20976(-3)$ & $7.21122(-3)$ & $-1.24108(-6)$ & $7.20997(-3)$ \\
30 & $1.63948(-2)$ & $2.70616(-6)$ & $1.63975(-2)$ & $1.64020(-2)$ & $-4.23978(-6)$ & $1.63978(-2)$ \\
40 & $2.94451(-2)$ & $9.29513(-6)$ & $2.94544(-2)$ & $2.94647(-2)$ & $-1.04395(-5)$ & $2.94543(-2)$ \\
50 & $4.65451(-2)$ & $1.82441(-5)$ & $4.65633(-2)$ & $4.65844(-2)$ & $-2.15904(-5)$ & $4.65628(-2)$ \\
60 & $6.79538(-2)$ & $2.79272(-5)$ & $6.79817(-2)$ & $6.80207(-2)$ & $-4.01290(-5)$ & $6.79806(-2)$ \\
70 & $9.40213(-2)$ & $3.57720(-5)$ & $9.40571(-2)$ & $9.41243(-2)$ & $-6.94242(-5)$ & $9.40549(-2)$ \\
80 & $1.25217(-1)$ & $3.81507(-5)$ & $1.25255(-1)$ & $1.25366(-1)$ & $-1.14086(-4)$ & $1.25252(-1)$ \\
90 & $1.62145(-1)$ & $3.02091(-5)$ & $1.62175(-1)$ & $1.62351(-1)$ & $-1.80357(-4)$ & $1.62171(-1)$ \\
100 & $2.05548(-1)$ & $5.64640(-6)$ & $2.05554(-1)$ & $2.05824(-1)$ & $-2.76660(-4)$ & $2.05547(-1)$ \\
& & & $2^{3} P_{2} \rightarrow 1^{1} S_{0}$ & & & \\
5 & 1.19578 & $1.08648(-5)$ & 1.19579 & 1.19579 & $-9.37185(-6)$ & 1.19578 \\
10 & $5.88007(-1)$ & $1.15662(-5)$ & $5.88019(-1)$ & $5.88025(-1)$ & $-7.24570(-6)$ & $5.88018(-1)$ \\
20 & $2.88201(-1)$ & $1.18155(-5)$ & $2.88213(-1)$ & $2.88219(-1)$ & $-6.36802(-6)$ & $2.88213(-1)$ \\
30 & $1.88203(-1)$ & $1.17979(-5)$ & $1.88215(-1)$ & $1.88221(-1)$ & $-6.03153(-6)$ & $1.88215(-1)$ \\
40 & $1.37464(-1)$ & $1.16429(-5)$ & $1.37476(-1)$ & $1.37481(-1)$ & $-5.84629(-6)$ & $1.37475(-1)$ \\
50 & $1.06295(-1)$ & $1.13411(-5)$ & $1.06306(-1)$ & $1.06311(-1)$ & $-5.79091(-6)$ & $1.06305(-1)$ \\
60 & $8.48580(-2)$ & $1.08573(-5)$ & $8.48689(-2)$ & $8.48745(-2)$ & $-5.90008(-6)$ & $8.48686(-2)$ \\
70 & $6.89535(-2)$ & $1.01491(-5)$ & $6.89636(-2)$ & $6.89700(-2)$ & $-6.22291(-6)$ & $6.89638(-2)$ \\
80 & $5.64791(-2)$ & $9.17127(-6)$ & $5.64883(-2)$ & $5.64948(-2)$ & $-6.81199(-6)$ & $5.64880(-2)$ \\
90 & $4.62644(-2)$ & $7.88189(-6)$ & $4.62723(-2)$ & $4.62797(-2)$ & $-7.71841(-6)$ & $4.62720(-2)$ \\
100 & $3.76016(-2)$ & $6.24541(-6)$ & $3.76078(-2)$ & $3.76165(-2)$ & $-8.98877(-6)$ & $3.76075(-2)$ \\
\hline \hline
\end{tabular}


interactions. By using the length gauge, we obtain amplitudes that are independent of the starting potential and that have negligible corrections from negative-energy states. In our calculations, we use theoretical energies obtained from the no-pair Hamiltonian that include QED corrections. As discussed in Refs. [3, 4], these energies are in very close agreement with experimental energies. Amplitudes for the M1 and M2 transitions are calculated in the velocity gauge. Negative-energy corrections, which are important in the velocity gauge, are obtained from second-order perturbation theory.

\subsection{Comparison with other theories}

Over the years, numerous nonrelativistic calculations of E1 amplitudes and rates have been made for the $2-1$ and $2-2$ transitions along the helium isoelectronic sequence. Among the most precise of these calculations are those of Schiff, Pekeris and Accad [21], who evaluated oscillator strengths of the $2{ }^{1} P \rightarrow 1{ }^{1} S$ and $2{ }^{3} P \rightarrow 2{ }^{3} S$ transitions for ions with nuclear charges in the range $Z=2-10$ using variational wave functions. These benchmark calculations were confirmed and extended by Kono and Hattori [22] and by Sandars and Knight [23]. Most recently, Cann and Thakkar [24] have carried out high-precision variational calculations of oscillator strengths for $S-P$ and $P-D$ transitions for ions in the range $Z=2-10$. The nonrelativistic oscillator strengths from Ref. [24] are rounded to five digits and compared with the present calculations in Table 7. The present results for the triplet transitions are obtained by summing the oscillator strengths of the three fine-structure components to give a composite relativistic oscillator strength. As can be seen from the table, the differences between the relativistic and nonrelativistic values range from one part in the fourth figure at $Z=2$ to three parts in the third figure at $Z=10$. This results in an error of about $0.4 \%$ in the nonrelativistic oscillator strength for the singlet transition and an error of $3 \%$ for the corresponding triplet transition at $Z=10$. The source of these errors is the use of nonrelativistic energies and wave functions in the calculations of Ref. [24].

Accurate nonrelativistic multi-configuration Hartree-Fock (MCHF) calculations of the oscillator strength of the $2^{3} P \rightarrow 2{ }^{3} S$ transition in $\mathrm{Li}^{+}(Z=3)$ were reported in Ref. [25]. These calculations gave close agreement with those of Refs. [21, 22, 23, 24] when nonrelativistic energies were used but came into agreement with the present calculation when relativistic corrections to the energy were made. Such energy corrections are ambiguous for triplet transitions and not at all possible for intercombination transitions.

Drake, in his unified model [26], devised a scheme for smoothly joining precise nonrelativistic calculations of the $2 P_{1} \rightarrow 1 S_{0}$ oscillator strengths at low $Z$ to uncorrelated, but relativistic, oscillator strengths at high $Z$. Using this method, accurate oscillator strengths for both the allowed transitions $2{ }^{1} P_{1} \rightarrow{ }^{1} S_{0}$ and the intercombination transitions $2{ }^{3} P_{1} \rightarrow 1{ }^{1} S_{0}$ were obtained throughout the isoelectronic sequence. In Table 8 , we compare the oscillator strengths from the unified model with values from the present calculation. For low values of $Z$, the unified model removes the discrepancies with the nonrelativistic variational 
Table 7: Comparison of the present relativistic calculations of oscillator strengths with the high-precision nonrelativistic calculations of Ref. [24].

\begin{tabular}{cccll}
\hline \hline & \multicolumn{2}{c}{$2{ }^{1} P \rightarrow 1{ }^{1} S$} & \multicolumn{2}{c}{$2{ }^{3} P \rightarrow 2{ }^{3} S$} \\
$Z$ & Non Rel. & Present & Non Rel. & Present \\
\hline 2 & 0.27617 & 0.2761 & 0.5391 & 0.5391 \\
3 & 0.45663 & 0.4565 & 0.30794 & 0.3081 \\
4 & 0.55156 & 0.5513 & 0.21314 & 0.2134 \\
5 & 0.60892 & 0.6085 & 0.16263 & 0.1632 \\
6 & 0.64707 & 0.6463 & 0.13138 & 0.1321 \\
7 & 0.67420 & 0.6731 & 0.11018 & 0.1112 \\
8 & 0.69445 & 0.6929 & 0.09486 & 0.09619 \\
9 & 0.71013 & 0.7080 & 0.08327 & 0.08595 \\
10 & 0.72262 & 0.7198 & 0.07420 & 0.07625 \\
\hline \hline
\end{tabular}

calculations, and for high $Z$ the unified model remains in agreement with the present calculations at the $1 \%$ level of accuracy. Similarly, the unified model gives oscillator strengths for the intercombination transitions that agree with the present calculations at the $1 \%$ level throughout the isoelectronic sequence, with the exception of very low values of $Z$. For the intercombination transition, our values disagree with those of Drake by more than $1 \%$ for $Z=2$, and agreement is not obtained until $Z=7$. We attribute this discrepancy to Drake's first-order treatment of the Breit interaction. While the Breit correction to the matrix element of the $2^{1} P_{1}-1{ }^{1} S_{0}$ transition is less than a percent of the total matrix element for the entire isoelectronic sequence, this is not so for the spin-forbidden transition. In fact, for $Z=2$, the Breit contribution to the matrix element is actually $52 \%$ of the total matrix element! This contribution drops to $12 \%$ for $Z=10$. Clearly for very low values of $Z$, the treatment of the Breit as a firstorder perturbation is inadequate. Since we include the Breit contribution to the matrix element exactly, within the numerical limits previously stated, our values of the rates for the $2^{3} P_{1}-1^{1} S_{0}$ transition are expected to be precise.

In addition to the unified model, a variety of other theoretical methods have been used to carry out relativistic calculations of transition probabilities along the helium isoelectronic sequence. Among these are the relativistic randomphase approximation (RRPA) [10], the multiconfiguration Dirac-Fock method (MCDF) [27], relativistic CI expansions [28], relativistic many-body theory [8], and QED perturbation theory $[9,29,30]$. In Table 9 we compare the decay rates predicted by the present calculation of the $2{ }^{1,3} P_{1} \rightarrow{ }^{1} S_{0}$ transitions with the relativistic calculations of Refs. $[10,26,28]$. All of the calculations agree at the few percent level for high values of $Z$, where correlation is unimportant. The $2{ }^{3} P_{1} \rightarrow 1{ }^{1} S_{0}$ rates at low values of $Z$ are very sensitive to correlation effects, leading to large errors in the approximate calculations of Refs. [10, 28]. The situation is similar for other E1 transitions; the allowed transition rates are de- 
Table 8: Comparison of the present relativistic calculations of oscillator strengths for $2{ }^{1} P_{1} \rightarrow 1{ }^{1} S_{0}$ and $2{ }^{3} P_{1} \rightarrow 1{ }^{1} S_{0}$ with the values from the unified model of Ref. [26].

\begin{tabular}{ccccc}
\hline \hline & \multicolumn{2}{c}{$2{ }^{1} P_{1} \rightarrow 1{ }^{1} S_{0}$} & \multicolumn{2}{c}{$2{ }^{3} P_{1} \rightarrow 1{ }^{1} S_{0}$} \\
$Z$ & Present & Unified & Present & Unified \\
\hline 2 & 0.2761 & 0.2762 & $2.810(-8)$ & $2.774(-8)$ \\
5 & 0.6085 & 0.6084 & $7.093(-6)$ & $7.082(-6)$ \\
10 & 0.7198 & 0.7196 & $4.425(-4)$ & $4.424(-4)$ \\
20 & 0.7454 & 0.7452 & $2.192(-2)$ & $2.193(-2)$ \\
30 & 0.6629 & 0.6628 & $1.080(-1)$ & $1.079(-1)$ \\
40 & 0.5743 & 0.5743 & $1.849(-1)$ & $1.847(-1)$ \\
50 & 0.5161 & 0.5162 & $2.216(-1)$ & $2.213(-1)$ \\
60 & 0.4727 & 0.4728 & $2.356(-1)$ & $2.352(-1)$ \\
70 & 0.4333 & 0.4332 & $2.382(-1)$ & $2.376(-1)$ \\
80 & 0.3932 & 0.3928 & $2.343(-1)$ & $2.335(-1)$ \\
90 & 0.3505 & 0.3493 & $2.260(-1)$ & $2.248(-1)$ \\
100 & 0.3042 & 0.3016 & $2.137(-1)$ & $2.119(-1)$ \\
\hline \hline
\end{tabular}

termined to within a few percent in the approximate calculations, but the more sensitive intercombination transitions are poorly described by the approximate calculations especially at low $Z$.

A great deal of attention has been given to the M1 transitions $2{ }^{3} S_{1} \rightarrow 1{ }^{1} S_{0}$ during the past twenty-five years. The interest stemmed from the identification of intense M1 lines in the spectrum of the sun by Gabriel and Jordan [31]. Calculations of M1 decay rate based on an expansion of the M1 matrix element in powers of $Z \alpha$ were carried out in Refs. [29, 32, 33], and other relativistic calculations were reported in Refs. [9, 10, 27, 28, 8, 30]. In Table 10, we compare the present calculations with some of these previous calculations. For low $Z$ ions, the approximate RRPA [10] and Krause [28] calculations are seriously in error because of inadequate treatment of correlation corrections. At intermediate $Z$, all M1 calculations agree to within $1 \%$. At high $Z$, the M1 amplitudes are sensitive to the atomic wave functions inside the nucleus; this has the consequence that the present M1 calculations, which include nuclear finite size corrections, deviate from the previous calculations, which were carried out for point nuclei, by two to three percent for ions with $Z>80$.

The calculation closest in spirit to the present one is that of Lindroth and Salomonson [8], who evaluated the M1 transition in $\mathrm{Ar}^{+16}$ using "exact" nopair wave functions. The Breit interaction and negative-energy corrections were treated perturbatively. The contributions to the M1 matrix element from positive- and negative-energy states in the two calculations are: 
Table 9: Comparison of the present E1 decay rates $\left(\mathrm{s}^{-1}\right)$ for the $2{ }^{1} P_{1} \rightarrow 1{ }^{1} S_{0}$ and $2{ }^{3} P_{1} \rightarrow 1{ }^{1} S_{0}$ transitions with other relativistic theories.

\begin{tabular}{clccl}
\hline \hline$Z$ & Present & Unified[26] & RRPA[10] & Krause[28] \\
\hline \multicolumn{5}{c}{$2{ }^{1} P_{1} \rightarrow 1{ }^{1} S_{0}$} \\
2 & $1.798(9)$ & $1.799(9)$ & $1.71(9)$ & $1.86(9)$ \\
5 & $3.719(11)$ & $3.719(11)$ & & $4.05(11)$ \\
10 & $8.850(12)$ & $8.851(12)$ & $8.85(12)$ & $9.28(12)$ \\
20 & $1.642(14)$ & $1.643(14)$ & $1.65(14)$ & $1.68(14)$ \\
30 & $7.764(14)$ & $7.773(14)$ & $7.81(14)$ & $7.88(14)$ \\
40 & $2.212(15)$ & $2.216(15)$ & & $2.23(15)$ \\
50 & $5.057(15)$ & $5.071(15)$ & $5.08(15)$ & $5.10(15)$ \\
60 & $1.006(16)$ & $1.010(16)$ & & $1.02(16)$ \\
70 & $1.805(16)$ & $1.813(16)$ & & $1.82(16)$ \\
80 & $2.986(16)$ & $3.000(16)$ & $3.01(16)$ & $3.02(16)$ \\
90 & $4.616(16)$ & $4.638(16)$ & & $4.68(16)$ \\
100 & $6.733(16)$ & $6.760(16)$ & $6.79(16)$ & $6.85(16)$ \\
& & $2{ }^{3} P_{1} \rightarrow 1{ }^{1} S_{0}$ & & \\
2 & $1.757(2)$ & $1.764(2)$ & $2.33(2)$ & $1.57(2)$ \\
5 & $4.210(6)$ & $4.220(6)$ & $4.36(6)$ & $4.15(6)$ \\
10 & $5.347(9)$ & $5.356(9)$ & $5.40(9)$ & $5.36(9)$ \\
20 & $4.777(12)$ & $4.786(12)$ & $4.85(12)$ & $4.93(12)$ \\
30 & $1.251(14)$ & $1.252(14)$ & $1.22(14)$ & $1.31(14)$ \\
40 & $7.008(14)$ & $7.017(14)$ & & $8.30(14)$ \\
50 & $2.120(15)$ & $2.123(15)$ & $2.12(15)$ & $2.19(15)$ \\
60 & $4.845(15)$ & $4.853(15)$ & & $4.99(15)$ \\
70 & $9.460(15)$ & $9.480(15)$ & & $9.72(15)$ \\
80 & $1.668(16)$ & $1.672(16)$ & $1.68(16)$ & $1.71(16)$ \\
90 & $2.732(16)$ & $2.741(16)$ & & $2.80(16)$ \\
100 & $4.231(16)$ & $4.250(16)$ & $4.27(16)$ & $4.34(16)$ \\
\hline \hline
\end{tabular}


Table 10: Comparison of the present $2{ }^{3} S_{1} \rightarrow 1{ }^{1} S_{0}$ M1 decay rate $\left(\mathrm{s}^{-1}\right)$ with other theories.

\begin{tabular}{clllll}
\hline \hline$Z$ & Present & Pert-Th.[9] & RRPA[10] & Drake[32] & Krause[28] \\
\hline 2 & $1.266(-4)$ & $1.253(-4)$ & $1.73(-4)$ & $1.272(-4)$ & $8.737(-5)$ \\
5 & 6.696 & 6.731 & 6.90 & 6.695 & 6.407 \\
10 & $1.092(4)$ & $1.098(4)$ & $1.10(4)$ & $1.087(4)$ & $1.081(4)$ \\
20 & $1.412(7)$ & $1.418(7)$ & $1.42(7)$ & $1.383(7)$ & $1.410(7)$ \\
30 & $8.981(8)$ & $9.023(8)$ & $9.01(8)$ & & $9.000(8)$ \\
40 & $1.719(10)$ & $1.729(10)$ & $1.73(10)$ & & $1.727(10)$ \\
50 & $1.726(11)$ & $1.737(11)$ & $1.74(11)$ & & $1.737(11)$ \\
60 & $1.161(12)$ & $1.171(12)$ & $1.17(12)$ & & $1.171(12)$ \\
70 & $5.968(12)$ & $6.032(12)$ & $6.03(12)$ & & $6.040(12)$ \\
80 & $2.540(13)$ & $2.577(13)$ & $2.58(13)$ & & $2.582(13)$ \\
90 & $9.439(13)$ & $9.639(13)$ & $9.63(13)$ & & $9.661(13)$ \\
100 & $3.181(14)$ & & $3.29(14)$ & & $3.299(14)$ \\
\hline \hline
\end{tabular}

\begin{tabular}{crr}
$10^{5} \times \mathrm{M}$ & Present & Ref.[8] \\
\hline$(+)$ & 2.1255 & 2.1226 \\
$(-)$ & -0.0004 & -0.0001 \\
Sum & 2.1251 & 2.1225 \\
\hline
\end{tabular}

The photon energy used in Ref. [8] was $\omega=114.072$ a.u., in good agreement with the value $\omega=114.077$ a.u. used in the present calculation. The lifetime of the $2{ }^{3} S_{1}$ state from Ref. [8] is $\tau=209.4 \pm 0.4 \mathrm{~ns}$, compared to the present value $\tau=208.9 \pm 0.2$ ns. The principal difference between the two calculations appears to be the treatment of the Breit interaction.

There have been various calculations of the $2{ }^{3} P_{2} \rightarrow 1{ }^{1} S_{0}$ M2 rate as well $[10,27,28,34,35]$. In Table 11, we compare our calculations of the M2 rate with some of these earlier calculations. We find excellent agreement for low $Z$ with the results of Drake [34] and for high $Z$ with the results of Krause [28].

\subsection{Comparison with experiment}

The precision of laboratory measurements of the $2{ }^{3} S_{1} \rightarrow 1{ }^{1} S_{0}$ (M1) transition rate has increased dramatically over the past few decades. Presently, the most accurately measured $\mathrm{M} 1$ rate is for $\mathrm{C}^{+4}$ [55]. The measured rate $A_{\exp }=48.57 \pm$ $0.11 \mathrm{~s}^{-1}$ differs with the present theoretical value $A_{\mathrm{th}}=48.60 \mathrm{~s}^{-1}$ by less than one standard deviation. The only other high-precision measurement of the $\mathrm{M} 1$ rate is for $\mathrm{Nb}^{39+}$ [57], where the measured rate differs from the present theoretical value by three standard deviations. Comparisons of the present calculations with other measured M1 rates are given in Table 12. The measured rates, which vary by fifteen orders of magnitude for $Z$ in the range $2-54$. are also compared with the present calculations in Fig. 3. 
Table 11: Comparison of the present $2{ }^{3} P_{2} \rightarrow 1{ }^{1} S_{0}$ M2 decay rate $\left(\mathrm{s}^{-1}\right)$ with other theories.

\begin{tabular}{ccllll}
\hline \hline$Z$ & Present & Drake [34] & RRPA [10] & Kundu [35] & Krause [28] \\
\hline 2 & $3.271(-1)$ & $3.27(-1)$ & $3.94(-1)$ & $3.62(-1)$ & $1.183(-1)$ \\
5 & $5.014(3)$ & $5.01(3)$ & $5.09(3)$ & $4.96(3)$ & $4.082(3)$ \\
10 & $2.257(6)$ & $2.26(6)$ & $2.27(6)$ & & $2.078(6)$ \\
20 & $7.510(8)$ & & $7.56(8)$ & & $7.254(8)$ \\
30 & $2.104(10)$ & & $2.12(10)$ & & $2.064(10)$ \\
40 & $2.208(11)$ & & $2.24(11)$ & & $2.187(11)$ \\
50 & $1.365(12)$ & & $1.39(12)$ & & $1.360(12)$ \\
60 & $6.060(12)$ & & $6.17(12)$ & & $6.070(12)$ \\
70 & $2.146(13)$ & & $2.19(13)$ & & $2.160(13)$ \\
80 & $6.457(13)$ & & $6.63(13)$ & & $6.529(13)$ \\
90 & $1.718(14)$ & & $1.77(14)$ & & $1.746(14)$ \\
100 & $4.156(14)$ & & $4.32(14)$ & & $4.254(14)$ \\
\hline
\end{tabular}

Table 12: Comparison of experimental M1 decay rates of the $2{ }^{3} S_{1}$ state $A^{\exp }$ with decay rates $A^{\text {theory }}$ from the present calculation.

\begin{tabular}{ccccc}
\hline \hline$Z$ & $A^{\text {exp }}$ & $A^{\text {theory }}$ & unit & Ref. \\
\hline 2 & $1.11 \pm 0.33$ & 1.266 & $10^{-4} \mathrm{~s}^{-1}$ & {$[47]$} \\
3 & $1.71 \pm 0.38$ & 2.035 & $10^{-2} \mathrm{~s}^{-1}$ & {$[49]$} \\
6 & $4.857 \pm 0.011$ & 4.860 & $10^{1} \mathrm{~s}^{-1}$ & {$[55]$} \\
7 & $2.561 \pm 0.031$ & 2.537 & $10^{2} \mathrm{~s}^{-1}$ & {$[55]$} \\
10 & $1.105 \pm 0.018$ & 1.092 & $10^{4} \mathrm{~s}^{-1}$ & {$[53]$} \\
16 & $1.41 \pm 0.17$ & 1.426 & $10^{6} \mathrm{~s}^{-1}$ & {$[48]$} \\
17 & $2.82 \pm 0.19$ & 2.661 & $10^{6} \mathrm{~s}^{-1}$ & {$[48]$} \\
18 & $4.93 \pm 0.29$ & 4.787 & $10^{6} \mathrm{~s}^{-1}$ & {$[50]$} \\
22 & $3.88 \pm 0.20$ & 3.750 & $10^{6} \mathrm{~s}^{-1}$ & {$[45]$} \\
23 & $5.92 \pm 0.24$ & 5.913 & $10^{6} \mathrm{~s}^{-1}$ & {$[46]$} \\
26 & $2.08 \pm 0.26$ & 2.075 & $10^{8} \mathrm{~s}^{-1}$ & {$[46]$} \\
35 & $4.46 \pm 0.14$ & 4.360 & $10^{9} \mathrm{~s}^{-1}$ & {$[52]$} \\
36 & $5.848 \pm 0.075$ & 5.822 & $10^{9} \mathrm{~s}^{-1}$ & {$[56]$} \\
41 & $2.1954 \pm 0.0068$ & 2.217 & $10^{10} \mathrm{~s}^{-1}$ & {$[57]$} \\
47 & $9.01 \pm 0.16$ & 9.083 & $10^{10} \mathrm{~s}^{-1}$ & {$[54]$} \\
54 & $3.92 \pm 0.12$ & 3.846 & $10^{11} \mathrm{~s}^{-1}$ & {$[51]$} \\
\hline \hline
\end{tabular}




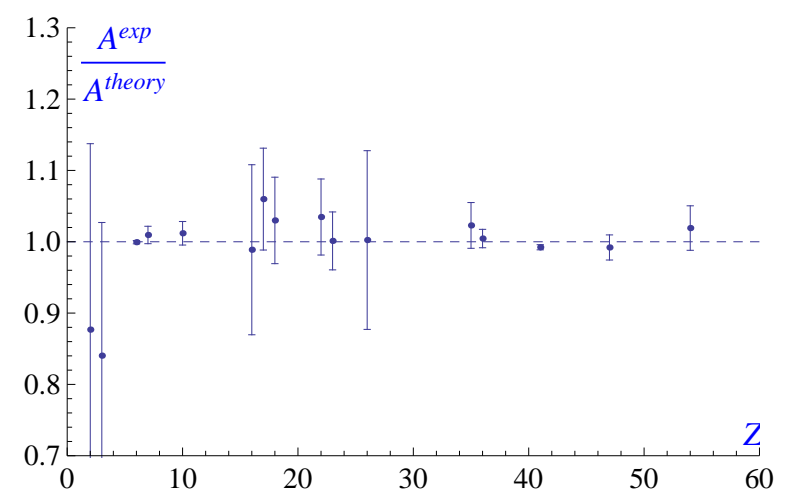

Figure 3: Ratio of experimental M1 decay rates of the $2{ }^{3} S_{1}$ state A ${ }^{\exp }$ to those from the present calculation $\mathrm{A}^{\text {theory }}$.

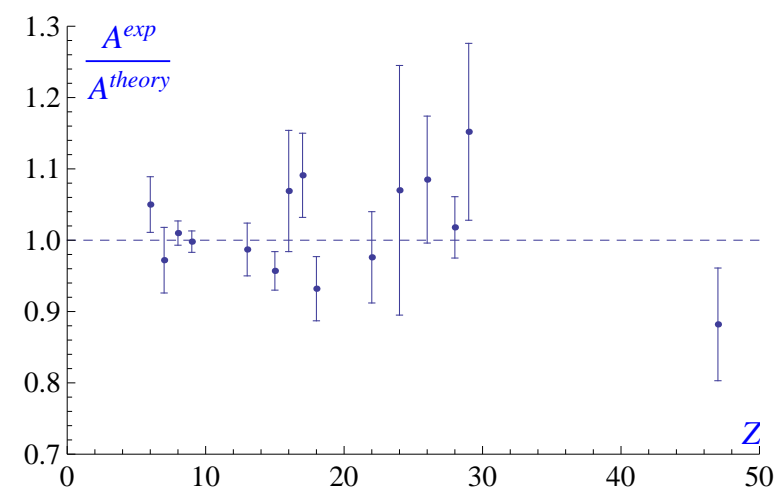

Figure 4: Ratio of experimental M2 + E1 decay rates of the $2{ }^{3} P_{2}$ state $\mathrm{A}^{\exp }$ to those from the present calculation $\mathrm{A}^{\text {theory }}$.

The decay of the $2{ }^{3} P_{2}$ state consists of two branches; $2{ }^{3} P_{2} \rightarrow 2{ }^{3} S_{1}$ (E1) and $2{ }^{3} P_{2} \rightarrow{ }^{1} S_{0}$ (M2). For those ions having nuclei with nonvanishing angular momenta, there is a third branch caused by hyperfine mixing of the $2{ }^{3} P_{2}$ state with the $2^{3} P_{1}$ state. This branch typically contributes only small corrections to the sum of the rates of the other two branches and will be ignored in the theoretical rates used here for comparison. For low values of $Z$, the E1 branch dominates, while for $Z \geq 19$, the M2 rate is larger. Although the lifetime measurements for the $2{ }^{3} P_{2}$ state are not as precise as those for the $2{ }^{3} S_{1}$ state, measurements at the $1.5 \%$ level of accuracy were carried out for $\mathrm{O}^{+6}$ and $\mathrm{F}^{+7}$ [66]. The resulting decay rates for both of these ions agree with the present calculation to within the experimental errors. Comparisons of experimental decay rates with the present calculations for M2 transitions are given in Table 13. and shown graphically in Fig. 4. 
Table 13: Comparison of experimental decay rates $A^{\exp }$ of the $2{ }^{3} P_{2}$ state with decay rates $A^{\text {th }}$ from the present calculation. Contributions to $A^{\text {theory }}$ from hyperfine mixing have been omitted.

\begin{tabular}{ccccc}
\hline \hline$Z$ & $A^{\exp }$ & $A^{\text {theory }}$ & unit & Ref. \\
\hline 6 & $5.99 \pm 0.22$ & 5.705 & $10^{7} \mathrm{~s}^{-1}$ & {$[71]$} \\
7 & $6.71 \pm 0.32$ & 6.901 & $10^{7} \mathrm{~s}^{-1}$ & {$[71]$} \\
8 & $8.26 \pm 0.14$ & 8.182 & $10^{7} \mathrm{~s}^{-1}$ & {$[66]$} \\
9 & $10.0 \pm 1.0$ & 9.598 & $10^{7} \mathrm{~s}^{-1}$ & {$[62]$} \\
& $9.58 \pm 0.14$ & 9.598 & $10^{7} \mathrm{~s}^{-1}$ & {$[66]$} \\
13 & $1.852 \pm 0.069$ & 1.877 & $10^{8} \mathrm{~s}^{-1}$ & {$[65]$} \\
15 & $2.778 \pm 0.077$ & 2.904 & $10^{8} \mathrm{~s}^{-1}$ & {$[67]$} \\
& $2.94 \pm 0.26$ & 2.904 & $10^{8} \mathrm{~s}^{-1}$ & {$[68]$} \\
16 & $4.00 \pm 0.32$ & 3.743 & $10^{8} \mathrm{~s}^{-1}$ & {$[59]$} \\
17 & $5.38 \pm 0.29$ & 4.933 & $10^{8} \mathrm{~s}^{-1}$ & {$[60]$} \\
18 & $5.9 \pm 1.0$ & 6.618 & $10^{8} \mathrm{~s}^{-1}$ & {$[58]$} \\
& $6.17 \pm 0.30$ & 6.618 & $10^{8} \mathrm{~s}^{-1}$ & {$[63]$} \\
22 & $2.27 \pm 0.15$ & 2.326 & $10^{9} \mathrm{~s}^{-1}$ & {$[63]$} \\
& $2.48 \pm 0.25$ & 2.326 & $10^{9} \mathrm{~s}^{-1}$ & {$[69]$} \\
24 & $4.65 \pm 0.76$ & 4.347 & $10^{9} \mathrm{~s}^{-1}$ & {$[69]$} \\
26 & $9.1 \pm 1.6$ & 7.929 & $10^{9} \mathrm{~s}^{-1}$ & {$[61]$} \\
& $8.9 \pm 1.0$ & 7.929 & $10^{9} \mathrm{~s}^{-1}$ & {$[64]$} \\
& $8.3 \pm 1.0$ & 7.929 & $10^{9} \mathrm{~s}^{-1}$ & {$[70]$} \\
28 & $1.429 \pm 0.061$ & 1.404 & $10^{10} \mathrm{~s}^{-1}$ & {$[73]$} \\
29 & $2.13 \pm 0.23$ & 1.849 & $10^{10} \mathrm{~s}^{-1}$ & {$[72]$} \\
47 & $8.06 \pm 0.72$ & 9.140 & $10^{11} \mathrm{~s}^{-1}$ & {$[74]$} \\
\hline \hline
\end{tabular}




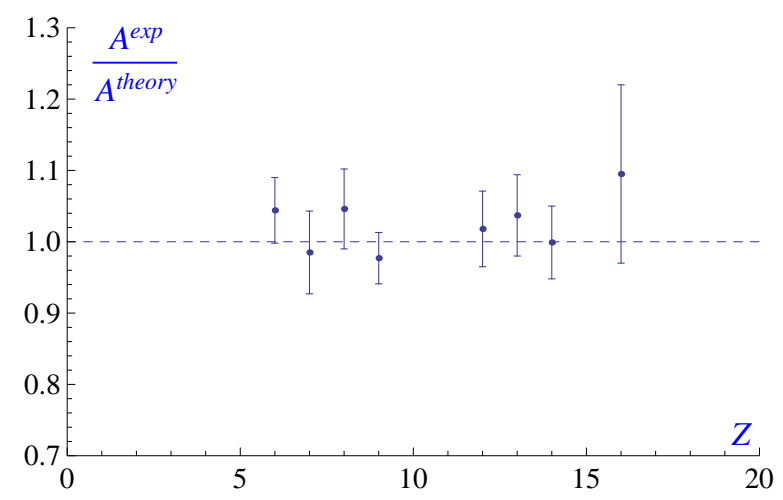

Figure 5: Ratio of experimental E1 decay rates of the $2{ }^{3} P_{1}$ state $\mathrm{A}^{\exp }$ to those from the present calculation $\mathrm{A}^{\text {theory }}$.

Table 14: Comparison of experimental E1 decay rates $A^{\exp }$ for $2{ }^{3} P_{1}$ states with the corresponding calculated decay rates $A^{\text {theory }}$, which are sums of the rates to the $2{ }^{3} S_{1}$ and $1{ }^{1} S_{0}$ states.

\begin{tabular}{ccccc}
\hline \hline$Z$ & $A^{\exp }$ & $A^{\text {theory }}$ & unit & Ref. \\
\hline 6 & $8.85 \pm 0.39$ & 8.478 & $10^{7} \mathrm{~s}^{-1}$ & {$[78]$} \\
7 & $2.04 \pm 0.12$ & 2.071 & $10^{8} \mathrm{~s}^{-1}$ & {$[78]$} \\
8 & $6.58 \pm 0.35$ & 6.292 & $10^{8} \mathrm{~s}^{-1}$ & {$[66]$} \\
9 & $1.88 \pm 0.07$ & 1.924 & $10^{9} \mathrm{~s}^{-1}$ & {$[66]$} \\
12 & $3.45 \pm 0.18$ & 3.388 & $10^{10} \mathrm{~s}^{-1}$ & {$[77]$} \\
13 & $7.81 \pm 0.43$ & 7.533 & $10^{10} \mathrm{~s}^{-1}$ & {$[77]$} \\
14 & $1.57 \pm 0.08$ & 1.572 & $10^{11} \mathrm{~s}^{-1}$ & {$[76]$} \\
16 & $6.37 \pm 0.73$ & 5.820 & $10^{11} \mathrm{~s}^{-1}$ & {$[76]$} \\
\hline \hline
\end{tabular}

Measurements of the rate of the intercombination transition $2{ }^{3} P_{1} \rightarrow{ }^{1} S_{0}$ (E1) transition at low values of $Z$ have also been made. These experimental measurements are compared with the present calculations in Table 14 and shown in Fig. 5.

\subsection{Compilation of transition rates}

Finally, in Table 15, we present a summary of our results for E1 transitions in the range $Z=2-100$, and in Table 16, we present a summary of our M1 and M2 calculations over the same range. In these tables, we list transition wavelengths $\lambda(\AA)$, transition multiplets, angular momenta of the initial and final states, Einstein A-coefficients, and line strengths $S$. Additionally, for E1 transitions, we tabulate (absorption) oscillator strengths $f$. The line strength $S$ (a.u.) is the square of the reduced matrix element of the multipole-moment 
operator. Thus,

$$
S= \begin{cases}\left|\left\langle F|| Q_{1}^{(1)}|| I\right\rangle\right|^{2}, & \text { for E1 transitions } \\ \left|\left\langle F|| M_{J}|| I\right\rangle\right|^{2}, & \text { for M1 and M2 transitions } .\end{cases}
$$

The Einstein A-coefficients $\left(\mathrm{s}^{-1}\right)$ listed in the tables were obtained in terms of line strengths using the relations

$$
\begin{aligned}
A_{k i} & =\frac{2.02613 \times 10^{18}}{\lambda^{3}\left[J_{k}\right]} S_{E 1}, \quad \text { for } \mathrm{E} 1 \text { transitions } \\
& =\frac{2.69735 \times 10^{13}}{\lambda^{3}\left[J_{k}\right]} S_{M 1}, \quad \text { for M1 transitions, } \\
& =\frac{1.49097 \times 10^{13}}{\lambda^{5}\left[J_{k}\right]} S_{M 2}, \quad \text { for M2 transitions }
\end{aligned}
$$

where $\left[J_{k}\right]=2 J_{k}+1$. Finally, the (dimensionless) oscillator strengths for E1 transitions are given in terms of E1 line strengths by

$$
f_{i k}=\frac{303.756}{\lambda\left[J_{i}\right]} S_{E 1} .
$$

In our numerical work, we have attempted to control the numerical accuracy of calculations of amplitudes to five significant figures. We believe that the transitions rates presented in Tables 15 and 16 are accurate to $0.1 \%$ or better from the numerical point of view.

\section{$5.4 \quad$ Future Prospects}

As discussed above, all of the transition rates listed in Tables 15 and 16 are expected to be accurate to better than $0.1 \%$. At this level of accuracy it becomes important to consider contributions to the rates from other sources. The starting point of our calculations, the no-pair Hamiltonian, does not by itself include the effects of the finite mass of the nucleus or QED, although the effect of finite nuclear size has been included. However, all of these effects have been included as completely as possible in our previous calculations of $n=1$ and $n=2$ energy levels in the helium isoelectronic sequence [3, 4]. By using these energies in the present calculation, we are partially accounting for recoil and QED. We now turn to the question of the importance of those recoil and QED corrections not accounted for by modifying the energies.

The highest-order calculations of radiative corrections to decay rates have been carried out for the exotic atom positronium [36]. The first-order corrections to the decay of orthopositronium are quite large [37],

$$
\Gamma^{1}=-10.2866(6) \frac{\alpha}{\pi} \Gamma^{0},
$$

a $2.4 \%$ correction. This correction has been confirmed experimentally [38], although a notable feature of the decay is that higher-order corrections must 
account for a $0.1 \%$ discrepancy between theory and experiment. Remarkably little is known about radiative corrections to decay rates of non-exotic atoms. Part of the reason for this is that, until recently, there has not been as much experimental impetus to carry out these difficult calculations. It is unusual to measure a decay rate at under the $1 \%$ level, and even corrections as large as those in orthopositronium would be difficult to detect for most decays. There are, however, important exceptions to this statement, most prominently in the case of heliumlike ions. As discussed above, the M1 decay of heliumlike carbon has been measured to $0.2 \%$ [55]. The agreement with our theory then definitely rules out a radiative correction as large as in orthopositronium, yet a correction of the order of the electron anomalous magnetic moment, $\alpha / 2 \pi$, which enters at the $0.1 \%$ level, cannot be ruled out. For the case of hydrogen, QED corrections have been treated in a general manner by Barbieri and Sucher [6] and for the specific case of M1 decays by Lin and Feinberg [39]. It was shown in the latter work that possible corrections of order $\alpha \ln \alpha$ and $\alpha$ vanish for the decay $2 S_{1 / 2} \rightarrow$ $1 S_{1 / 2}$ in hydrogenic ions. This result appears to be specific to hydrogen, and the more complicated heliumlike calculation has not been carried out. Inasmuch as at high $Z$, heliumlike ions can be treated as perturbations to the hydrogenic case, a possible $\alpha / Z$ correction may be expected, which will be beyond our claimed accuracy. In addition to the leading order $\alpha$ corrections, there are also corrections of order $\alpha(Z \alpha)^{2}$, which could enter at the $0.1 \%$ level at high $Z$ depending on the coefficient. We note that while the E1 transition rates are in general poorly known, a recent high precision $(0.5 \%)$ measurement of lifetimes of $3^{3} S_{1}$ and $3^{3} D_{J}$ levels in neutral helium [40] agrees with the calculations of Kono and Hattori [22]. Again, while this rules out large radiative corrections, smaller corrections could easily be present.

Another high-accuracy experiment that sheds light on the size of radiative corrections is the measurement of the $\mathrm{He}^{+}$lifetime by Drake, Kwela, and Wijngaarden [41]. This experiment quotes an error of $0.075 \%$, and agrees with a theoretical decay rate that does not include QED. This again rules out a large coefficient of $\alpha / \pi$, and is close to ruling out even an $\alpha / 2 \pi$ correction. Finally, we note that a number of experiments accurate at the few tenths of a percent level have been carried out on alkali atoms [42, 43, 44], some of which disagree with theory by almost a percent. Because of the different electronic configuration, we do not see that the small radiative correction inferred from $\mathrm{He}^{+}$need carry over to these atoms, and believe it possible that larger radiative corrections may be present in the case of the alkalis. In any case, it seems clear that the theory of radiative corrections to atomic decay rates is, with the exception of orthopositronium, a relatively unexplored field of QED. It cannot be overemphasized that experiments of the highest possible accuracy are essential for stimulating progress on these demanding calculations. What we hope we have provided in the present paper is a set of calculations of such high accuracy that any deviation from such experiments can be unambiguously interpreted as due to radiative corrections. We consider the complete calculation of radiative corrections the main theoretical challenge remaining for our theoretical understanding of decay rates of helium and heliumlike ions. 
This work of DP and JS was supported by NSF grant PHY-92-04089. The work of WRJ was supported in part by NSF grant PHY-92-04089 and in part by the Alexander von Humboldt foundation at the Technical University of Dresden. The authors owe thanks to Y.-K. Kim, P. Mohr and G. Soff for useful suggestions, and to A.E. Livingston and H.G. Berry for a careful reading of the manuscript. Thanks are also due to J. Fuhr for helpful discussions on the NIST database.

\section{Useful identities}

In this appendix, we prove that the commutator $[V, \Xi]$ vanishes if the sum over intermediate states in Eq.(49) is extended to include negative-energy states. As before, we may write the potential operator as

$$
\begin{aligned}
V & =\frac{1}{2} \sum_{i j k l} v_{i j k l} a_{i}^{\dagger} a_{j}^{\dagger} a_{l} a_{k}, \\
\Xi & =\sum_{p q} \xi_{p q} a_{p}^{\dagger} a_{q},
\end{aligned}
$$

where

$$
v_{i j k l}=\left\langle i j\left|V\left(\mathbf{x}_{1}, \mathbf{x}_{2}\right)\right| k l\right\rangle .
$$

Here $V$ is the sum of the Coulomb and instantaneous Breit interactions, and

$$
\xi_{i j}=\langle i|\xi(\mathbf{x})| j\rangle .
$$

The sums are restricted to positive-energy states only. With some algebra, the commutator can be put in the form

$$
[V, \Xi]=\frac{1}{2} \sum_{i j k l} O_{i j k l} a_{i}^{\dagger} a_{j}^{\dagger} a_{l} a_{k},
$$

where

$$
O_{i j k l}=\sum_{r}\left\{v_{i j r l} \xi_{r k}+v_{i j k r} \xi_{r l}-\xi_{i r} v_{r j k l}-\xi_{j r} v_{i r k l}\right\} .
$$

Let us introduce the positive-energy projection operator

$$
\Lambda_{+}\left(\mathbf{x}, \mathbf{x}^{\prime}\right)=\sum_{r} \psi_{r}(\mathbf{x}) \psi_{r}^{\dagger}\left(\mathbf{x}^{\prime}\right) .
$$

We can then write Eq.(98) as

$$
O_{i j k l}=\int d^{3} x_{1} d^{3} x_{2} d^{3} x_{3} d^{3} x_{4} \psi_{i}^{\dagger}\left(\mathbf{x}_{1}\right) \psi_{j}^{\dagger}\left(\mathbf{x}_{2}\right) O\left(\mathbf{x}_{1}, \mathbf{x}_{2} ; \mathbf{x}_{3}, \mathbf{x}_{4}\right) \psi_{k}\left(\mathbf{x}_{3}\right) \psi_{l}\left(\mathbf{x}_{4}\right),
$$

with

$$
\begin{aligned}
O\left(\mathbf{x}_{1}, \mathbf{x}_{2} ; \mathbf{x}_{3}, \mathbf{x}_{4}\right) & =\Lambda_{+}\left(\mathbf{x}_{1}, \mathbf{x}_{3}\right) \delta\left(\mathbf{x}_{2}, \mathbf{x}_{4}\right)\left[V\left(\mathbf{x}_{1}, \mathbf{x}_{2}\right) \xi\left(\mathbf{x}_{3}\right)-\xi\left(\mathbf{x}_{1}\right) V\left(\mathbf{x}_{3}, \mathbf{x}_{4}\right)\right] \\
& +\Lambda_{+}\left(\mathbf{x}_{2}, \mathbf{x}_{4}\right) \delta\left(\mathbf{x}_{1}, \mathbf{x}_{3}\right)\left[V\left(\mathbf{x}_{1}, \mathbf{x}_{2}\right) \xi\left(\mathbf{x}_{4}\right)-\xi\left(\mathbf{x}_{2}\right) V\left(\mathbf{x}_{3}, \mathbf{x}_{4}\right)[101)\right.
\end{aligned}
$$


If the sum in Eq.(99) is extended over both positive- and negative-energy states, then $\Lambda_{+}\left(\mathbf{x}, \mathbf{x}^{\prime}\right) \rightarrow \delta\left(\mathbf{x}, \mathbf{x}^{\prime}\right)$, and it follows from Eq.(101) that $O\left(\mathbf{x}_{1}, \mathbf{x}_{2} ; \mathbf{x}_{3}, \mathbf{x}_{4}\right)=$ 0 . This, in turn, implies $[V, \Xi]=0$.

In a similar way, the terms on the first two lines of Eq.(84) in Sec. 4.1 can be shown to vanish if the sum over intermediate states $i$ extends over both positiveand negative-energy states.

\section{References}

[1] J. Sucher, Phys. Rev. A22, 348 (1980).

[2] M.H. Mittleman, Phys. Rev. A4, 893 (1971); Phys. Rev. A5, 2395 (1972); Phys. Rev. A24, 1167 (1981).

[3] M.H. Chen, K.T. Cheng, and W.R. Johnson, Phys. Rev. A47, 3692 (1993).

[4] D.R. Plante, W.R. Johnson, and J. Sapirstein, Phys. Rev. A49, 3519 (1994).

[5] J. Hiller, J. Sucher, G. Feinberg, and B. Lynn, Ann. Phys. (N.Y.) 127, 149 (1980).

[6] R. Barbieri and J. Sucher, Nucl. Phys. B 134, 155 (1978). See also P.J. Mohr, in Relativistic, Quantum Electrodynamic, and Weak Interaction Effects in Atoms, Ed. Walter Johnson, Peter Mohr, and Joseph Sucher, AIP Conference Proceeding 189 (AIP, New York, 1989).

[7] M. Gell-Mann and F. Low, Phys. Rev. 84350 (1951).

[8] Eva Lindroth and Sten Salomonson, Phys. Rev. A 41, 4659 (1990).

[9] W.R. Johnson and Chein-ping Lin, Phys. Rev. A9, 1486 (1974).

[10] W.R. Johnson and C.D. Lin, Phys. Rev. A 14, 565 (1976); C.D. Lin, W.R. Johnson, and A. Dalgarno, Phys. Rev. A 15, 154 (1977).

[11] W.R. Johnson, S.A. Blundell, and J. Sapirstein, Phys. Rev. A 37, 2764 (1988).

[12] A.I. Akhiezer and V.B. Berestetskii, Quantum Electrodynamics (Interscience, New York, 1965), p. 28.

[13] B.W. Shore and D.H. Menzel, Principles of Atomic Spectra (Wiley, New York, 1968), p. 435; M. Mizushima, Quantum Mechanics of Atomic Spectra and Atomic Structure (W.A. Benjamin, New York, 1970), p. 88; I.I. Sobelman, Atomic Spectra and Radiative Transitions (Springer, Berlin, 1992), Sec. 9.3.

[14] See, for example, J.R. Fuhr, G.A. Martin, and W.L. Wiese, J. Phys. Chem. Ref. Data 17, Suppl. 4 (1988). 
[15] P. Indelicato and P.J. Mohr, Theor. Chem. Acta 80, 207 (1991).

[16] J. Sucher, Phys. Rev. 107, 1448 (1957).

[17] A. Desiderio and W.R. Johnson, Phys. Rev. A 3, 1267 (1971).

[18] W.H. Furry, Phys. Rev. 81, 115 (1951).

[19] E. Avgoustoglou, W.R. Johnson, D.R. Plante, J. Sapirstein, S. Sheinerman, and S.A. Blundell, Phys. Rev. A 46, 5478 (1992).

[20] W.R. Johnson and G. Soff, At. Data. Nucl. Data Tables 33, 405 (1985).

[21] B. Schiff, C.L. Pekeris, and Y. Accad, Phys. Rev. A 4, 885 (1971).

[22] A. Kono and S. Hattori, Phys. Rev. A 29, 2981 (1984).

[23] F.C. Sanders and R.F. Knight, Phys. Rev. A 39, 4387 (1989).

[24] N.M. Cann and A.J. Thakkar, Phys. Rev. A 46, 5397 (1992).

[25] C. Froese Fischer, Nucl. Instrum. Methods Phy. Res., B 31, 265 (1988).

[26] G.W.F. Drake, Phys. Rev. A 19, 1387 (1979).

[27] J. Hata and I.P. Grant, J. Phys. B 14, 2111 (1981).

[28] J. Krause, Phys. Rev. A 34, 3692 (1986).

[29] G. Feinberg and J. Sucher, Phys. Rev. Lett. 26, 681 (1971).

[30] R. Marrus and P.J. Mohr, Adv. At. Mol. Phys. 14, 181 (1978).

[31] A.H. Gabriel and Carol Jordan, Nature 221, 947 (1969); Mon. Not. R. Astron. Soc. 145, 241 (1969); Phys. Lett. A 32, 166 (1970).

[32] G.W.F. Drake, Phys. Rev. A 3, 908 (1971).

[33] I.L. Beigman and U.I. Safronova, Zh. Eksp. Teor. Fiz. 60, 2045 (1971) [Sov. Phys._JETP 33, 1102 (1971)].

[34] G.W.F. Drake, Ap. J. 158, 1199 (1969); G.W.F. Drake, Ap. J. 163, 439 (1971).

[35] B. Kundu, P.K. Mukherjee, and H.P. Roy, Phys. Scr. 39, 722 (1989).

[36] P. Labelle, G.P. Lepage, and U. Magnea, Phys. Rev. Lett. 72, 2006 (1994).

[37] G.S. Adkins, A.A. Salahuddin, and K.E. Schalm, Phys. Rev. A 45, 7774 (1992).

[38] J.S. Nico, D.W. Gidley, A. Rich, and P.W. Zitzewitz, Phys. Rev. Lett. 65, 1344 (1990). 
[39] D.L. Lin and G. Feinberg, Phys. Rev. A 10, 1425 (1974).

[40] U. Volz, D. Marger, H. Roth, and H. Schmoranzer, University of Kaiserslautern preprint (1995).

[41] G.W.F. Drake, J. Kwela, and A. van Wijngaarden, Phys. Rev. A 46, 113 (1992).

[42] A. Gaupp, R. Kuske, and H.J. Andraä, Phys. Rev. A 26, 3351 (1982).

[43] C. Tanner, Atomic Physics 14, ed. D.J. Wineland, C.E. Wieman, and S.J. Smith (AIP Press, New York 1995), p. 130.

[44] J. Jin and D.A. Church, Phys. Rev. A 49, 3463 (1994).

[45] H. Gould, R. Marrus, and R.W. Schmieder, Phys. Rev. Lett. 31, 504 (1973).

[46] H. Gould, R. Marrus, and P.J. Mohr, Phys. Rev. Lett. 33, 676 (1974).

[47] H.W. Moos and J.R. Woodworth, Phys. Rev. A 12, 2455 (1975).

[48] J.A. Bednar, C.L. Cocke, B. Curnutte, and R. Randall, Phys. Rev. A 11, 460 (1975).

[49] R.D. Knight and M.H. Prior, Phys. Rev. A 21, 179 (1980).

[50] G. Hurbricht amd E. Träbert, Z. Phys. D 7, 243 (1987).

[51] R. Marrus, P. Charles, P. Indelicato, L. de Billy, C. Tazi, J.P. Briand, A. Simionovici, D.D. Dietrich, F. Bosch, D. Liesen, Phys. Rev. A 39, 3725 (1989).

[52] R.W. Dunford, D.A. Church, C.J. Liu, H.G. Berry, M.L. Raphaelian, M. Hass, and L.J. Curtis, Phys. Rev. A 41, 4109 (1990).

[53] B.J. Wargelin, P. Beiersdorfer, S.M. Kahn, Phys. Rev. Lett. 71, 2196 (1993).

[54] B.B. Birkett, J.P. Briand, P. Charles, D.D. Dietrich, K. Finlayson, P. Indelicato, D. Liesen, R. Marrus, and A. Simionovici, Phys. Rev. A 47, R2454 (1993).

[55] H.T. Schmidt, P. Forck, M. Grieser, D. Habs, J. Kenntner, G. Miersch, R. Repnow, U. Schramm, T. Schüssler, D. Schwalm, A. Wolf, Phys. Rev. Lett. 72, 1616 (1994).

[56] S. Cheng, R.W. Dunford, C.J. Liu, B.J. Zabransky, A.E. Livingston, and L.J. Curtis, Phys. Rev. A 49, 2347 (1994).

[57] A. Simionovici, B.B. Birkett, R. Marrus, P. Charles, P. Indelicato, D.D. Dietrich, and K. Finlayson, Phys. Rev. A 49, 3553 (1994). 
[58] R. Marrus and R.W. Schmieder, Phys. Rev. A 5, 1160 (1972).

[59] C.L. Cocke, B. Curnutte, and R. Randall, Phys. Rev. A 9, 1823 (1974).

[60] C.L. Cocke, B. Curnutte, J.R. MacDonald, and R. Randall, Phys. Rev. A 9, 57 (1974).

[61] H. Gould, R. Marrus, and P.J. Mohr, Phys. Rev. Lett. 33, 676 (1974).

[62] J.R. Mowat, P.M. Griffin, H.H. Haselton, R. Laubert, D.J. Pegg, R.S. Peterson, I.A. Sellin, and R.S. Thoe, Phys. Rev. A 11, 2198 (1975).

[63] W.A. Davis and R. Marrus, Phys. Rev. A 15, 1963 (1977).

[64] R. Marrus and P.J. Mohr, Adv. At. Mol. Phys. 14, 181 (1978).

[65] B. Denne, S. Huldt, J. Pihl, and R. Hallin, Phys. Scr. 22, 45 (1980).

[66] L. Engström, C. Jupén, B. Denne, S. Huldt, W.T. Meng, P. Kaijser, J.O. Ekberg, U. Litzén, and I. Martinson, Phys. Scr. 22, 570 (1981).

[67] Ph. Deschepper, P. Lebrun, L. Palffy, and P. Pellegrin, Phys. Rev. A 26, 1271 (1982).

[68] A.E. Livingston and S.J. Hinterlong, Nucl. Instrum. Methods 202, 103 (1982).

[69] H.D. Dohmann, R. Mann, and E. Pfeng, Z. Phys. A 309, 101 (1982).

[70] J.P. Buchet, M.C. Buchet-Poulizac, A. Denis, J. Désesquelles, M. Dreutta, J.P. Grandin, M. Huet, X. Husson, and D. Lecler, Phys. Rev. A 30, 309 (1984).

[71] R. Hutton, N. Reistad, L. Engström, S. Huldt, Phys. Scr. 31, 506 (1985).

[72] J.P. Buchet, M.C. Buchet-Poulizac, A. Denis, J. Désesquelles, M. Dreutta, J.P. Grandin, X. Husson, D. Lecler, and H.F. Beyer, Nucl. Instrum. Methods B 9, 645 (1985).

[73] R.W. Dunford, C.J. Liu, J. Last, N. Berrah-Mansour, R. Vondrasek, D.A. Church, and L.J. Curtis, Phys. Rev. A 44, 764 (1991).

[74] A. Simionovici, B.B. Birkett, J.P. Briand, P. Charles, D.D. Dietrich, K. Finlayson, P. Indelicato, D. Liesen, and R. Marrus, Phys. Rev. A 48, 1695 (1993).

[75] P. Richard, R.L. Kauffman, F. Hopkins, C.W. Woods, and K.A. Jamison, Phys. Rev. A 8, 2187 (1973).

[76] S. L. Varghese, C. L. Cocke, and B. Curnutte, Phys. Rev. A 14, 1729 (1976). 
[77] I. A. Armour, J.D. Silver, and E. Träbert, J. Phys. B 14, 3563 (1981).

[78] R. Hutton, N. Reistad, L. Engström, and S. Huldt, Phys. Scr. 31, 506 (1985). 
Table 15: E1 transitions

\begin{tabular}{|c|c|c|c|c|c|c|c|}
\hline \multicolumn{3}{|c|}{ Transition } & \multicolumn{4}{|c|}{$A_{k i}$} & \multirow[b]{2}{*}{ S (a.u.) } \\
\hline$Z$ & Array & Multiplet & $\lambda(\AA)$ & $J_{i}-J_{k}$ & $\left(s^{-1}\right)$ & $f_{i k}$ & \\
\hline \multirow[t]{7}{*}{2} & \multirow[t]{2}{*}{$1 s^{2}-1 s 2 p$} & ${ }^{1} S-{ }^{3} P$ & 591.33 & $0-1$ & $1.787(2)$ & $2.810(-8)$ & $5.4698(-8)$ \\
\hline & & ${ }^{1} S-{ }^{1} P$ & 584.25 & $0-1$ & $1.799(9)$ & $2.761(-1)$ & $5.3110(-1)$ \\
\hline & \multirow[t]{5}{*}{$1 s 2 s-1 s 2 p$} & ${ }^{3} S-{ }^{3} P$ & 10832. & $1-2$ & $1.022(7)$ & $2.995(-1)$ & $3.2038(1)$ \\
\hline & & & 10832. & $1-1$ & $1.022(7)$ & $1.797(-1)$ & $1.9223(1)$ \\
\hline & & & 10831. & $1-0$ & $1.022(7)$ & $5.990(-2)$ & 6.4077 \\
\hline & & ${ }^{3} S-{ }^{1} P$ & 8864.8 & $1-1$ & 1.552 & $1.828(-8)$ & $1.6006(-6)$ \\
\hline & & ${ }^{1} S-{ }^{1} P$ & 20584 . & $0-1$ & $1.976(6)$ & $1.255(-1)$ & $2.5516(1)$ \\
\hline \multirow[t]{7}{*}{3} & \multirow[t]{2}{*}{$1 s^{2}-1 s 2 p$} & ${ }^{1} S-{ }^{3} P$ & 202.30 & $0-1$ & $1.790(4)$ & $3.295(-7)$ & $2.1945(-7)$ \\
\hline & & ${ }^{1} S-{ }^{1} P$ & 199.26 & $0-1$ & $2.556(10)$ & $4.565(-1)$ & $2.9947(-1)$ \\
\hline & \multirow[t]{5}{*}{$1 s 2 s-1 s 2 p$} & ${ }^{3} S-{ }^{3} P$ & 5485.6 & $1-2$ & $2.276(7)$ & $1.712(-1)$ & 9.2730 \\
\hline & & & 5486.2 & $1-1$ & $2.276(7)$ & $1.027(-1)$ & 5.5637 \\
\hline & & & 5484.7 & $1-0$ & $2.278(7)$ & $3.424(-2)$ & 1.8546 \\
\hline & & ${ }^{3} S-{ }^{1} P$ & 3879.7 & $1-1$ & $4.190(1)$ & $9.456(-8)$ & $3.6232(-6)$ \\
\hline & & ${ }^{1} S-{ }^{1} P$ & 9583.4 & $0-1$ & $5.148(6)$ & $7.088(-2)$ & 6.7089 \\
\hline \multirow[t]{7}{*}{4} & \multirow[t]{2}{*}{$1 s^{2}-1 s 2 p$} & ${ }^{1} S-{ }^{3} P$ & 101.68 & $0-1$ & $4.002(5)$ & $1.861(-6)$ & $6.2305(-7)$ \\
\hline & & ${ }^{1} S-{ }^{1} P$ & 100.25 & $0-1$ & $1.220(11)$ & $5.513(-1)$ & $1.8194(-1)$ \\
\hline & \multirow[t]{5}{*}{$1 s 2 s-1 s 2 p$} & ${ }^{3} S-{ }^{3} P$ & 3721.7 & $1-2$ & $3.427(7)$ & $1.186(-1)$ & 4.3597 \\
\hline & & & 3723.7 & $1-1$ & $3.421(7)$ & $7.112(-2)$ & 2.6156 \\
\hline & & & 3722.1 & $1-0$ & $3.426(7)$ & $2.372(-2)$ & $8.7187(-1)$ \\
\hline & & ${ }^{3} S-{ }^{1} P$ & 2441.7 & $1-1$ & $3.853(2)$ & $3.444(-7)$ & $8.3054(-6)$ \\
\hline & & ${ }^{1} S-{ }^{1} P$ & 6143.3 & $0-1$ & $8.757(6)$ & $4.955(-2)$ & 3.0063 \\
\hline \multirow[t]{7}{*}{5} & \multirow[t]{2}{*}{$1 s^{2}-1 s 2 p$} & ${ }^{1} S-{ }^{3} P$ & 61.086 & $0-1$ & $4.226(6)$ & $7.093(-6)$ & $1.4264(-6)$ \\
\hline & & ${ }^{1} S-{ }^{1} P$ & 60.311 & $0-1$ & $3.719(11)$ & $6.085(-1)$ & $1.2081(-1)$ \\
\hline & \multirow[t]{5}{*}{$1 s 2 s-1 s 2 p$} & ${ }^{3} S-{ }^{3} P$ & 2822.3 & $1-2$ & $4.557(7)$ & $9.070(-2)$ & 2.5281 \\
\hline & & & 2826.5 & $1-1$ & $4.536(7)$ & $5.433(-2)$ & 1.5165 \\
\hline & & & 2825.2 & $1-0$ & $4.542(7)$ & $1.812(-2)$ & $5.0551(-1)$ \\
\hline & & ${ }^{3} S-{ }^{1} P$ & 1771.9 & $1-1$ & $2.061(3)$ & $9.701(-7)$ & $1.6977(-5)$ \\
\hline & & ${ }^{1} S-{ }^{1} P$ & 4491.8 & $0-1$ & $1.262(7)$ & $3.818(-2)$ & 1.6936 \\
\hline \multirow[t]{7}{*}{6} & \multirow[t]{2}{*}{$1 s^{2}-1 s 2 p$} & ${ }^{1} S-{ }^{3} P$ & 40.728 & $0-1$ & $2.830(7)$ & $2.111(-5)$ & $2.8309(-6)$ \\
\hline & & ${ }^{1} S-{ }^{1} P$ & 40.266 & $0-1$ & $8.864(11)$ & $6.463(-1)$ & $8.5679(-2)$ \\
\hline & \multirow[t]{5}{*}{$1 s 2 s-1 s 2 p$} & ${ }^{3} S-{ }^{3} P$ & 2271.5 & $1-2$ & $5.702(7)$ & $7.352(-2)$ & 1.6492 \\
\hline & & & 2278.5 & $1-1$ & $5.648(7)$ & $4.396(-2)$ & $9.8919(-1)$ \\
\hline & & & 2277.9 & $1-0$ & $5.652(7)$ & $1.466(-2)$ & $3.2971(-1)$ \\
\hline & & ${ }^{3} S-{ }^{1} P$ & 1386.7 & $1-1$ & $7.952(3)$ & $2.293(-6)$ & $3.1400(-5)$ \\
\hline & & ${ }^{1} S-{ }^{1} P$ & 3527.4 & $0-1$ & $1.668(7)$ & $3.111(-2)$ & 1.0839 \\
\hline
\end{tabular}


Table 15: E1 transitions

\begin{tabular}{|c|c|c|c|c|c|c|c|}
\hline \multicolumn{3}{|c|}{ Transition } & \multicolumn{4}{|c|}{$A_{k i}$} & \multirow[b]{2}{*}{ S (a.u.) } \\
\hline$Z$ & Array & Multiplet & $\lambda(\AA)$ & $J_{i}-J_{k}$ & $\left(s^{-1}\right)$ & $f_{i k}$ & \\
\hline \multirow[t]{7}{*}{7} & \multirow[t]{2}{*}{$1 s^{2}-1 s 2 p$} & ${ }^{1} S-{ }^{3} P$ & 29.083 & $0-1$ & $1.394(8)$ & $5.303(-5)$ & $5.0772(-6)$ \\
\hline & & ${ }^{1} S-{ }^{1} P$ & 28.786 & $0-1$ & $1.806(12)$ & $6.731(-1)$ & $6.3789(-2)$ \\
\hline & \multirow[t]{5}{*}{$1 s 2 s-1 s 2 p$} & ${ }^{3} S-{ }^{3} P$ & 1896.7 & $1-2$ & $6.891(7)$ & $6.195(-2)$ & 1.1604 \\
\hline & & & 1907.3 & $1-1$ & $6.774(7)$ & $3.694(-2)$ & $6.9586(-1)$ \\
\hline & & & 1907.6 & $1-0$ & $6.770(7)$ & $1.231(-2)$ & $2.3194(-1)$ \\
\hline & & ${ }^{3} S-{ }^{1} P$ & 1137.1 & $1-1$ & $2.465(4)$ & $4.778(-6)$ & $5.3656(-5)$ \\
\hline & & ${ }^{1} S-{ }^{1} P$ & 2896.0 & $0-1$ & $2.092(7)$ & $2.630(-2)$ & $7.5225(-1)$ \\
\hline \multirow[t]{7}{*}{8} & \multirow[t]{2}{*}{$1 s^{2}-1 s 2 p$} & ${ }^{1} S-{ }^{3} P$ & 21.803 & $0-1$ & $5.499(8)$ & $1.176(-4)$ & $8.4392(-6)$ \\
\hline & & ${ }^{1} S-{ }^{1} P$ & 21.601 & $0-1$ & $3.302(12)$ & $6.929(-1)$ & $4.9275(-2)$ \\
\hline & \multirow[t]{5}{*}{$1 s 2 s-1 s 2 p$} & ${ }^{3} S-{ }^{3} P$ & 1623.6 & $1-2$ & $8.149(7)$ & $5.368(-2)$ & $8.6073(-1)$ \\
\hline & & & 1638.3 & $1-1$ & $7.925(7)$ & $3.189(-2)$ & $5.1599(-1)$ \\
\hline & & & 1639.9 & $1-0$ & $7.902(7)$ & $1.062(-2)$ & $1.7198(-1)$ \\
\hline & & ${ }^{3} S-{ }^{1} P$ & 961.95 & $1-1$ & $6.533(4)$ & $9.063(-6)$ & $8.6099(-5)$ \\
\hline & & ${ }^{1} S-{ }^{1} P$ & 2449.6 & $0-1$ & $2.537(7)$ & $2.283(-2)$ & $5.5223(-1)$ \\
\hline \multirow[t]{7}{*}{9} & \multirow[t]{2}{*}{$1 s^{2}-1 s 2 p$} & ${ }^{1} S-{ }^{3} P$ & 16.950 & $0-1$ & $1.833(9)$ & $2.369(-4)$ & $1.3219(-5)$ \\
\hline & & ${ }^{1} S-{ }^{1} P$ & 16.806 & $0-1$ & $5.574(12)$ & $7.080(-1)$ & $3.9173(-2)$ \\
\hline & \multirow[t]{5}{*}{$1 s 2 s-1 s 2 p$} & ${ }^{3} S-{ }^{3} P$ & 1414.4 & $1-2$ & $9.506(7)$ & $4.752(-2)$ & $6.6375(-1)$ \\
\hline & & & 1433.8 & $1-1$ & $9.113(7)$ & $2.809(-2)$ & $3.9774(-1)$ \\
\hline & & & 1436.9 & $1-0$ & $9.054(7)$ & $9.342(-3)$ & $1.3258(-1)$ \\
\hline & & ${ }^{3} S-{ }^{1} P$ & 832.19 & $1-1$ & $1.539(5)$ & $1.598(-5)$ & $1.3130(-4)$ \\
\hline & & ${ }^{1} S-{ }^{1} P$ & 2116.1 & $0-1$ & $3.011(7)$ & $2.021(-2)$ & $4.2245(-1)$ \\
\hline \multirow[t]{7}{*}{10} & \multirow[t]{2}{*}{$1 s^{2}-1 s 2 p$} & ${ }^{1} S-{ }^{3} P$ & 13.553 & $0-1$ & $5.356(9)$ & $4.425(-4)$ & $1.9742(-5)$ \\
\hline & & ${ }^{1} S-{ }^{1} P$ & 13.447 & $0-1$ & $8.851(12)$ & $7.198(-1)$ & $3.1865(-2)$ \\
\hline & \multirow[t]{5}{*}{$1 s 2 s-1 s 2 p$} & ${ }^{3} S-{ }^{3} P$ & 1248.1 & $1-2$ & $1.099(8)$ & $4.278(-2)$ & $5.2738(-1)$ \\
\hline & & & 1272.8 & $1-1$ & $1.034(8)$ & $2.513(-2)$ & $3.1585(-1)$ \\
\hline & & & 1277.7 & $1-0$ & $1.023(8)$ & $8.344(-3)$ & $1.0530(-1)$ \\
\hline & & ${ }^{3} S-{ }^{1} P$ & 731.96 & $1-1$ & $3.307(5)$ & $2.656(-5)$ & $1.9200(-4)$ \\
\hline & & ${ }^{1} S-{ }^{1} P$ & 1856.3 & $0-1$ & $3.521(7)$ & $1.819(-2)$ & $3.3345(-1)$ \\
\hline \multirow[t]{7}{*}{11} & \multirow[t]{2}{*}{$1 s^{2}-1 s 2 p$} & ${ }^{1} S-{ }^{3} P$ & 11.083 & $0-1$ & $1.405(10)$ & $7.764(-4)$ & $2.8328(-5)$ \\
\hline & & ${ }^{1} S-{ }^{1} P$ & 11.003 & $0-1$ & $1.339(13)$ & $7.291(-1)$ & $2.6408(-2)$ \\
\hline & \multirow[t]{5}{*}{$1 s 2 s-1 s 2 p$} & ${ }^{3} S-{ }^{3} P$ & 1111.8 & $1-2$ & $1.265(8)$ & $3.908(-2)$ & $4.2907(-1)$ \\
\hline & & & 1142.3 & $1-1$ & $1.163(8)$ & $2.276(-2)$ & $2.5678(-1)$ \\
\hline & & & 1149.2 & $1-0$ & $1.143(8)$ & $7.544(-3)$ & $8.5629(-2)$ \\
\hline & & ${ }^{3} S-{ }^{1} P$ & 652.03 & $1-1$ & $6.600(5)$ & $4.207(-5)$ & $2.7089(-4)$ \\
\hline & & ${ }^{1} S-{ }^{1} P$ & 1647.1 & $0-1$ & $4.077(7)$ & $1.658(-2)$ & $2.6976(-1)$ \\
\hline \multirow[t]{2}{*}{12} & $1 s^{2}-1 s 2 p$ & ${ }^{1} S-{ }^{3} P$ & 9.2310 & $0-1$ & $3.375(10)$ & $1.293(-3)$ & $3.9307(-5)$ \\
\hline & & & & & & \multicolumn{2}{|c|}{ Continued on next page } \\
\hline
\end{tabular}


Table 15: E1 transitions

\begin{tabular}{|c|c|c|c|c|c|c|c|}
\hline \multicolumn{3}{|c|}{ Transition } & \multicolumn{4}{|c|}{$A_{k i}$} & \multirow[b]{2}{*}{ S (a.u.) } \\
\hline$Z$ & Array & Multiplet & $\lambda(\AA)$ & $J_{i}-J_{k}$ & $\left(s^{-1}\right)$ & $f_{i k}$ & \\
\hline & & ${ }^{1} S-{ }^{1} P$ & 9.1685 & $0-1$ & $1.948(13)$ & $7.364(-1)$ & $2.2227(-2)$ \\
\hline & $1 s 2 s-1 s 2 p$ & ${ }^{3} S-{ }^{3} P$ & 997.46 & $1-2$ & $1.453(8)$ & $3.612(-2)$ & $3.5587(-1)$ \\
\hline & & & 1034.3 & $1-1$ & $1.299(8)$ & $2.083(-2)$ & $2.1275(-1)$ \\
\hline & & & 1043.3 & $1-0$ & $1.266(8)$ & $6.889(-3)$ & $7.0983(-2)$ \\
\hline & & ${ }^{3} S-{ }^{1} P$ & 586.59 & $1-1$ & $1.240(6)$ & $6.399(-5)$ & $3.7069(-4)$ \\
\hline & & ${ }^{1} S-{ }^{1} P$ & 1473.9 & $0-1$ & $4.696(7)$ & $1.529(-2)$ & $2.2262(-1)$ \\
\hline \multirow[t]{7}{*}{13} & $1 s^{2}-1 s 2 p$ & ${ }^{1} S-{ }^{3} P$ & 7.8068 & $0-1$ & $7.519(10)$ & $2.061(-3)$ & $5.2970(-5)$ \\
\hline & & ${ }^{1} S-{ }^{1} P$ & 7.7571 & $0-1$ & $2.742(13)$ & $7.420(-1)$ & $1.8950(-2)$ \\
\hline & $1 s 2 s-1 s 2 p$ & ${ }^{3} S-{ }^{3} P$ & 899.65 & $1-2$ & $1.669(8)$ & $3.375(-2)$ & $2.9990(-1)$ \\
\hline & & & 943.16 & $1-1$ & $1.441(8)$ & $1.922(-2)$ & $1.7903(-1)$ \\
\hline & & & 954.38 & $1-0$ & $1.393(8)$ & $6.343(-3)$ & $5.9784(-2)$ \\
\hline & & ${ }^{3} S-{ }^{1} P$ & 531.85 & $1-1$ & $2.217(6)$ & $9.400(-5)$ & $4.9377(-4)$ \\
\hline & & ${ }^{1} S-{ }^{1} P$ & 1327.3 & $0-1$ & $5.393(7)$ & $1.424(-2)$ & $1.8673(-1)$ \\
\hline \multirow[t]{7}{*}{14} & $1 s^{2}-1 s 2 p$ & ${ }^{1} S-{ }^{3} P$ & 6.6881 & $0-1$ & $1.570(11)$ & $3.159(-3)$ & $6.9556(-5)$ \\
\hline & & ${ }^{1} S-{ }^{1} P$ & 6.6478 & $0-1$ & $3.755(13)$ & $7.463(-1)$ & $1.6333(-2)$ \\
\hline & $1 s 2 s-1 s 2 p$ & ${ }^{3} S-{ }^{3} P$ & 814.70 & $1-2$ & $1.919(8)$ & $3.183(-2)$ & $2.5614(-1)$ \\
\hline & & & 865.15 & $1-1$ & $1.592(8)$ & $1.786(-2)$ & $1.5262(-1)$ \\
\hline & & & 878.65 & $1-0$ & $1.524(8)$ & $5.880(-3)$ & $5.1029(-2)$ \\
\hline & & ${ }^{3} S-{ }^{1} P$ & 485.22 & $1-1$ & $3.796(6)$ & $1.340(-4)$ & $6.4205(-4)$ \\
\hline & & ${ }^{1} S-{ }^{1} P$ & 1200.9 & $0-1$ & $6.191(7)$ & $1.339(-2)$ & $1.5876(-1)$ \\
\hline \multirow[t]{7}{*}{15} & $1 s^{2}-1 s 2 p$ & ${ }^{1} S-{ }^{3} P$ & 5.7933 & $0-1$ & $3.099(11)$ & $4.678(-3)$ & $8.9226(-5)$ \\
\hline & & ${ }^{1} S-{ }^{1} P$ & 5.7601 & $0-1$ & $5.021(13)$ & $7.492(-1)$ & $1.4208(-2)$ \\
\hline & $1 s 2 s-1 s 2 p$ & ${ }^{3} S-{ }^{3} P$ & 739.90 & $1-2$ & $2.214(8)$ & $3.028(-2)$ & $2.2128(-1)$ \\
\hline & & & 797.52 & $1-1$ & $1.751(8)$ & $1.670(-2)$ & $1.3153(-1)$ \\
\hline & & & 813.29 & $1-0$ & $1.659(8)$ & $5.485(-3)$ & $4.4055(-2)$ \\
\hline & & ${ }^{3} S-{ }^{1} P$ & 444.88 & $1-1$ & $6.265(6)$ & $1.859(-4)$ & $8.1675(-4)$ \\
\hline & & ${ }^{1} S-{ }^{1} P$ & 1090.1 & $0-1$ & $7.118(7)$ & $1.268(-2)$ & $1.3651(-1)$ \\
\hline \multirow[t]{7}{*}{16} & $1 s^{2}-1 s 2 p$ & ${ }^{1} S-{ }^{3} P$ & 5.0664 & $0-1$ & $5.818(11)$ & $6.717(-3)$ & $1.1203(-4)$ \\
\hline & & ${ }^{1} S-{ }^{1} P$ & 5.0386 & $0-1$ & $6.576(13)$ & $7.509(-1)$ & $1.2456(-2)$ \\
\hline & $1 s 2 s-1 s 2 p$ & ${ }^{3} S-{ }^{3} P$ & 673.41 & $1-2$ & $2.562(8)$ & $2.903(-2)$ & $1.9307(-1)$ \\
\hline & & & 738.33 & $1-1$ & $1.920(8)$ & $1.569(-2)$ & $1.1440(-1)$ \\
\hline & & & 756.31 & $1-0$ & $1.799(8)$ & $5.142(-3)$ & $3.8410(-2)$ \\
\hline & & ${ }^{3} S-{ }^{1} P$ & 409.48 & $1-1$ & $1.001(7)$ & $2.517(-4)$ & $1.0181(-3)$ \\
\hline & & ${ }^{1} S-{ }^{1} P$ & 991.61 & $0-1$ & $8.208(7)$ & $1.210(-2)$ & $1.1850(-1)$ \\
\hline \multirow[t]{3}{*}{17} & $1 s^{2}-1 s 2 p$ & ${ }^{1} S-{ }^{3} P$ & 4.4679 & $0-1$ & $1.044(12)$ & $9.374(-3)$ & $1.3788(-4)$ \\
\hline & & ${ }^{1} S-{ }^{1} P$ & 4.4444 & $0-1$ & $8.458(13)$ & $7.514(-1)$ & $1.0994(-2)$ \\
\hline & & & & & & \multicolumn{2}{|c|}{ Continued on next page } \\
\hline
\end{tabular}


Table 15: E1 transitions

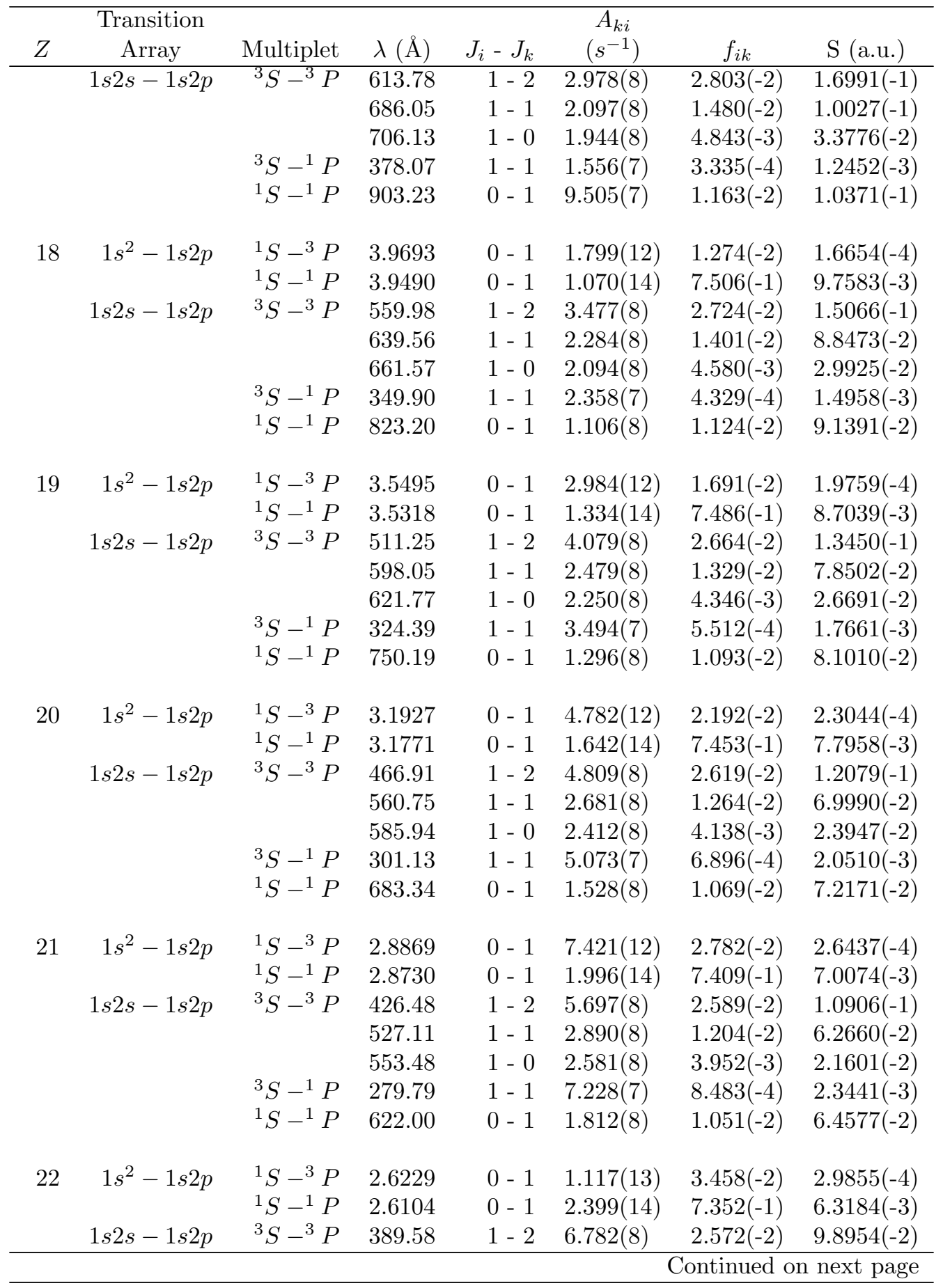


Table 15: E1 transitions

\begin{tabular}{|c|c|c|c|c|c|c|c|}
\hline \multicolumn{3}{|c|}{ Transition } & \multicolumn{4}{|c|}{$A_{k i}$} & \multirow[b]{2}{*}{ S (a.u.) } \\
\hline$Z$ & Array & Multiplet & $\lambda(\AA)$ & $J_{i}-J_{k}$ & $\left(s^{-1}\right)$ & $f_{i k}$ & \\
\hline \multirow{11}{*}{23} & & & 496.70 & $1-1$ & $3.103(8)$ & $1.148(-2)$ & $5.6299(-2)$ \\
\hline & & & 523.97 & $1-0$ & $2.758(8)$ & $3.783(-3)$ & $1.9578(-2)$ \\
\hline & & ${ }^{3} S-{ }^{1} P$ & 260.09 & $1-1$ & $1.013(8)$ & $1.027(-3)$ & $2.6384(-3)$ \\
\hline & & ${ }^{1} S-{ }^{1} P$ & 565.58 & $0-1$ & $2.165(8)$ & $1.038(-2)$ & $5.8002(-2)$ \\
\hline & $1 s^{2}-1 s 2 p$ & ${ }^{1} S-{ }^{3} P$ & 2.3933 & $0-1$ & $1.636(13)$ & $4.215(-2)$ & $3.3211(-4)$ \\
\hline & & ${ }^{1} S-{ }^{1} P$ & 2.3819 & $0-1$ & $2.855(14)$ & $7.285(-1)$ & $5.7127(-3)$ \\
\hline & $1 s 2 s-1 s 2 p$ & ${ }^{3} S-{ }^{3} P$ & 355.86 & $1-2$ & $8.109(8)$ & $2.566(-2)$ & $9.0179(-2)$ \\
\hline & & & 468.96 & $1-1$ & $3.323(8)$ & $1.096(-2)$ & $5.0745(-2)$ \\
\hline & & & 497.02 & $1-0$ & $2.941(8)$ & $3.631(-3)$ & $1.7822(-2)$ \\
\hline & & ${ }^{3} S-{ }^{1} P$ & 241.85 & $1-1$ & $1.397(8)$ & $1.225(-3)$ & $2.9262(-3)$ \\
\hline & & ${ }^{1} S-{ }^{1} P$ & 513.84 & $0-1$ & $2.602(8)$ & $1.030(-2)$ & $5.2271(-2)$ \\
\hline \multirow[t]{7}{*}{24} & $1 s^{2}-1 s 2 p$ & ${ }^{1} S-{ }^{3} P$ & 2.1925 & $0-1$ & $2.334(13)$ & $5.046(-2)$ & $3.6419(-4)$ \\
\hline & & ${ }^{1} S-{ }^{1} P$ & 2.1820 & $0-1$ & $3.366(14)$ & $7.208(-1)$ & $5.1780(-3)$ \\
\hline & $1 s 2 s-1 s 2 p$ & ${ }^{3} S-{ }^{3} P$ & 325.06 & $1-2$ & $9.735(8)$ & $2.570(-2)$ & $8.2513(-2)$ \\
\hline & & & 444.07 & $1-1$ & $3.538(8)$ & $1.046(-2)$ & $4.5870(-2)$ \\
\hline & & & 472.24 & $1-0$ & $3.134(8)$ & $3.492(-3)$ & $1.6288(-2)$ \\
\hline & & ${ }^{3} S-{ }^{1} P$ & 224.85 & $1-1$ & $1.901(8)$ & $1.441(-3)$ & $3.2003(-3)$ \\
\hline & & ${ }^{1} S-{ }^{1} P$ & 466.10 & $0-1$ & $3.151(8)$ & $1.026(-2)$ & $4.7251(-2)$ \\
\hline \multirow[t]{7}{*}{25} & $1 s^{2}-1 s 2 p$ & ${ }^{1} S-{ }^{3} P$ & 2.0158 & $0-1$ & $3.249(13)$ & $5.937(-2)$ & $3.9400(-4)$ \\
\hline & & ${ }^{1} S-{ }^{1} P$ & 2.0061 & $0-1$ & $3.935(14)$ & $7.123(-1)$ & $4.7041(-3)$ \\
\hline & $1 s 2 s-1 s 2 p$ & ${ }^{3} S-{ }^{3} P$ & 296.91 & $1-2$ & $1.173(9)$ & $2.584(-2)$ & $7.5776(-2)$ \\
\hline & & & 421.24 & $1-1$ & $3.756(8)$ & $9.993(-3)$ & $4.1573(-2)$ \\
\hline & & & 449.42 & $1-0$ & $3.335(8)$ & $3.366(-3)$ & $1.4940(-2)$ \\
\hline & & ${ }^{3} S-{ }^{1} P$ & 209.02 & $1-1$ & $2.554(8)$ & $1.673(-3)$ & $3.4541(-3)$ \\
\hline & & ${ }^{1} S-{ }^{1} P$ & 422.45 & $0-1$ & $3.837(8)$ & $1.027(-2)$ & $4.2833(-2)$ \\
\hline \multirow[t]{7}{*}{26} & $1 s^{2}-1 s 2 p$ & ${ }^{1} S-{ }^{3} P$ & 1.8595 & $0-1$ & $4.421(13)$ & $6.876(-2)$ & $4.2092(-4)$ \\
\hline & & ${ }^{1} S-{ }^{1} P$ & 1.8504 & $0-1$ & $4.566(14)$ & $7.031(-1)$ & $4.2830(-3)$ \\
\hline & $1 s 2 s-1 s 2 p$ & ${ }^{3} S-{ }^{3} P$ & 271.19 & $1-2$ & $1.419(9)$ & $2.607(-2)$ & $6.9824(-2)$ \\
\hline & & & 400.42 & $1-1$ & $3.973(8)$ & $9.551(-3)$ & $3.7772(-2)$ \\
\hline & & & 428.32 & $1-0$ & $3.545(8)$ & $3.250(-3)$ & $1.3749(-2)$ \\
\hline & & ${ }^{3} S-{ }^{1} P$ & 194.26 & $1-1$ & $3.392(8)$ & $1.919(-3)$ & $3.6822(-3)$ \\
\hline & & ${ }^{1} S-{ }^{1} P$ & 382.55 & $0-1$ & $4.697(8)$ & $1.030(-2)$ & $3.8931(-2)$ \\
\hline \multirow[t]{5}{*}{27} & $1 s^{2}-1 s 2 p$ & ${ }^{1} S-{ }^{3} P$ & 1.7206 & $0-1$ & $5.893(13)$ & $7.846(-2)$ & $4.4445(-4)$ \\
\hline & & ${ }^{1} S-{ }^{1} P$ & 1.7120 & $0-1$ & $5.260(14)$ & $6.934(-1)$ & $3.9080(-3)$ \\
\hline & $1 s 2 s-1 s 2 p$ & ${ }^{3} S-{ }^{3} P$ & 247.71 & $1-2$ & $1.721(9)$ & $2.638(-2)$ & $6.4540(-2)$ \\
\hline & & & 381.36 & $1-1$ & $4.189(8)$ & $9.134(-3)$ & $3.4402(-2)$ \\
\hline & & & & & & \multicolumn{2}{|c|}{ Continued on next page } \\
\hline
\end{tabular}


Table 15: E1 transitions

\begin{tabular}{|c|c|c|c|c|c|c|c|}
\hline \multicolumn{3}{|c|}{ Transition } & \multicolumn{4}{|c|}{$A_{k i}$} & \multirow[b]{2}{*}{ S (a.u.) } \\
\hline$Z$ & Array & Multiplet & $\lambda(\AA)$ & $J_{i}-J_{k}$ & $\left(s^{-1}\right)$ & $f_{i k}$ & \\
\hline \multirow{10}{*}{28} & & & 408.74 & $1-0$ & $3.765(8)$ & $3.144(-3)$ & $1.2691(-2)$ \\
\hline & & ${ }^{3} S-{ }^{1} P$ & 180.47 & $1-1$ & $4.459(8)$ & $2.177(-3)$ & $3.8807(-3)$ \\
\hline & & ${ }^{1} S-{ }^{1} P$ & 346.15 & $0-1$ & $5.777(8)$ & $1.038(-2)$ & $3.5476(-2)$ \\
\hline & \multirow[t]{2}{*}{$1 s^{2}-1 s 2 p$} & ${ }^{1} S-{ }^{3} P$ & 1.5966 & $0-1$ & $7.705(13)$ & $8.834(-2)$ & $4.6429(-4)$ \\
\hline & & ${ }^{1} S-{ }^{1} P$ & 1.5884 & $0-1$ & $6.022(14)$ & $6.833(-1)$ & $3.5733(-3)$ \\
\hline & \multirow[t]{5}{*}{$1 s 2 s-1 s 2 p$} & ${ }^{3} S-{ }^{3} P$ & 226.29 & $1-2$ & $2.092(9)$ & $2.677(-2)$ & $5.9827(-2)$ \\
\hline & & & 363.90 & $1-1$ & $4.402(8)$ & $8.739(-3)$ & $3.1407(-2)$ \\
\hline & & & 390.53 & $1-0$ & $3.996(8)$ & $3.046(-3)$ & $1.1748(-2)$ \\
\hline & & ${ }^{3} S-{ }^{1} P$ & 167.60 & $1-1$ & $5.806(8)$ & $2.445(-3)$ & $4.0471(-3)$ \\
\hline & & ${ }^{1} S-{ }^{1} P$ & 313.06 & $0-1$ & $7.134(8)$ & $1.048(-2)$ & $3.2408(-2)$ \\
\hline \multirow[t]{7}{*}{29} & \multirow[t]{2}{*}{$1 s^{2}-1 s 2 p$} & ${ }^{1} S-{ }^{3} P$ & 1.4854 & $0-1$ & $9.899(13)$ & $9.823(-2)$ & $4.8033(-4)$ \\
\hline & & ${ }^{1} S-{ }^{1} P$ & 1.4776 & $0-1$ & $6.855(14)$ & $6.731(-1)$ & $3.2743(-3)$ \\
\hline & \multirow[t]{5}{*}{$1 s 2 s-1 s 2 p$} & ${ }^{3} S-{ }^{3} P$ & 206.76 & $1-2$ & $2.549(9)$ & $2.723(-2)$ & $5.5606(-2)$ \\
\hline & & & 347.86 & $1-1$ & $4.611(8)$ & $8.365(-3)$ & $2.8740(-2)$ \\
\hline & & & 373.54 & $1-0$ & $4.239(8)$ & $2.956(-3)$ & $1.0904(-2)$ \\
\hline & & ${ }^{3} S-{ }^{1} P$ & 155.58 & $1-1$ & $7.498(8)$ & $2.721(-3)$ & $4.1806(-3)$ \\
\hline & & ${ }^{1} S-{ }^{1} P$ & 283.05 & $0-1$ & $8.839(8)$ & $1.062(-2)$ & $2.9680(-2)$ \\
\hline \multirow[t]{7}{*}{30} & \multirow[t]{2}{*}{$1 s^{2}-1 s 2 p$} & ${ }^{1} S-{ }^{3} P$ & 1.3853 & $0-1$ & $1.251(14)$ & $1.080(-1)$ & $4.9257(-4)$ \\
\hline & & ${ }^{1} S-{ }^{1} P$ & 1.3778 & $0-1$ & $7.763(14)$ & $6.629(-1)$ & $3.0067(-3)$ \\
\hline & \multirow[t]{5}{*}{$1 s 2 s-1 s 2 p$} & ${ }^{3} S-{ }^{3} P$ & 188.96 & $1-2$ & $3.112(9)$ & $2.776(-2)$ & $5.1810(-2)$ \\
\hline & & & 333.09 & $1-1$ & $4.818(8)$ & $8.013(-3)$ & $2.6361(-2)$ \\
\hline & & & 357.65 & $1-0$ & $4.493(8)$ & $2.872(-3)$ & $1.0144(-2)$ \\
\hline & & ${ }^{3} S-{ }^{1} P$ & 144.37 & $1-1$ & $9.610(8)$ & $3.003(-3)$ & $4.2815(-3)$ \\
\hline & & ${ }^{1} S-{ }^{1} P$ & 255.90 & $0-1$ & $1.098(9)$ & $1.078(-2)$ & $2.7248(-2)$ \\
\hline \multirow[t]{7}{*}{31} & \multirow[t]{2}{*}{$1 s^{2}-1 s 2 p$} & ${ }^{1} S-{ }^{3} P$ & 1.2949 & $0-1$ & $1.559(14)$ & $1.176(-1)$ & $5.0116(-4)$ \\
\hline & & ${ }^{1} S-{ }^{1} P$ & 1.2877 & $0-1$ & $8.752(14)$ & $6.527(-1)$ & $2.7669(-3)$ \\
\hline & \multirow[t]{5}{*}{$1 s 2 s-1 s 2 p$} & ${ }^{3} S-{ }^{3} P$ & 172.74 & $1-2$ & $3.804(9)$ & $2.836(-2)$ & $4.8385(-2)$ \\
\hline & & & 319.46 & $1-1$ & $5.021(8)$ & $7.682(-3)$ & $2.4239(-2)$ \\
\hline & & & 342.74 & $1-0$ & $4.760(8)$ & $2.794(-3)$ & $9.4586(-3)$ \\
\hline & & ${ }^{3} S-{ }^{1} P$ & 133.92 & $1-1$ & $1.223(9)$ & $3.290(-3)$ & $4.3510(-3)$ \\
\hline & & ${ }^{1} S-{ }^{1} P$ & 231.37 & $0-1$ & $1.367(9)$ & $1.097(-2)$ & $2.5077(-2)$ \\
\hline \multirow[t]{6}{*}{32} & \multirow[t]{2}{*}{$1 s^{2}-1 s 2 p$} & ${ }^{1} S-{ }^{3} P$ & 1.2131 & $0-1$ & $1.916(14)$ & $1.268(-1)$ & $5.0640(-4)$ \\
\hline & & ${ }^{1} S-{ }^{1} P$ & 1.2060 & $0-1$ & $9.824(14)$ & $6.427(-1)$ & $2.5517(-3)$ \\
\hline & \multirow[t]{4}{*}{$1 s 2 s-1 s 2 p$} & ${ }^{3} S-{ }^{3} P$ & 157.98 & $1-2$ & $4.654(9)$ & $2.902(-2)$ & $4.5283(-2)$ \\
\hline & & & 306.84 & $1-1$ & $5.221(8)$ & $7.370(-3)$ & $2.2336(-2)$ \\
\hline & & & 328.74 & $1-0$ & $5.040(8)$ & $2.722(-3)$ & $8.8379(-3)$ \\
\hline & & & & & & \multicolumn{2}{|c|}{ Continued on next page } \\
\hline
\end{tabular}


Table 15: E1 transitions

\begin{tabular}{|c|c|c|c|c|c|c|c|}
\hline \multicolumn{3}{|c|}{ Transition } & \multicolumn{4}{|c|}{$A_{k i}$} & \multirow[b]{2}{*}{ S (a.u.) } \\
\hline$Z$ & Array & Multiplet & $\lambda(\AA)$ & $J_{i}-J_{k}$ & $\left(s^{-1}\right)$ & $f_{i k}$ & \\
\hline \multirow{9}{*}{33} & & ${ }^{3} S-{ }^{1} P$ & 124.19 & $1-1$ & $1.549(9)$ & $3.581(-3)$ & $4.3921(-3)$ \\
\hline & & ${ }^{1} S-{ }^{1} P$ & 209.27 & $0-1$ & $1.705(9)$ & $1.119(-2)$ & $2.3136(-2)$ \\
\hline & $1 s^{2}-1 s 2 p$ & ${ }^{1} S-{ }^{3} P$ & 1.1386 & $0-1$ & $2.327(14)$ & $1.357(-1)$ & $5.0858(-4)$ \\
\hline & & ${ }^{1} S-{ }^{1} P$ & 1.1318 & $0-1$ & $1.099(15)$ & $6.329(-1)$ & $2.3582(-3)$ \\
\hline & $1 s 2 s-1 s 2 p$ & ${ }^{3} S-{ }^{3} P$ & 144.55 & $1-2$ & $5.697(9)$ & $2.974(-2)$ & $4.2465(-2)$ \\
\hline & & & 295.15 & $1-1$ & $5.419(8)$ & $7.077(-3)$ & $2.0630(-2)$ \\
\hline & & & 315.55 & $1-0$ & $5.335(8)$ & $2.655(-3)$ & $8.2741(-3)$ \\
\hline & & ${ }^{3} S-{ }^{1} P$ & 115.14 & $1-1$ & $1.950(9)$ & $3.875(-3)$ & $4.4070(-3)$ \\
\hline & & ${ }^{1} S-{ }^{1} P$ & 189.38 & $0-1$ & $2.128(9)$ & $1.144(-2)$ & $2.1396(-2)$ \\
\hline \multirow[t]{7}{*}{34} & $1 s^{2}-1 s 2 p$ & ${ }^{1} S-{ }^{3} P$ & 1.0707 & $0-1$ & $2.795(14)$ & $1.441(-1)$ & $5.0802(-4)$ \\
\hline & & ${ }^{1} S-{ }^{1} P$ & 1.0641 & $0-1$ & $1.224(15)$ & $6.235(-1)$ & $2.1840(-3)$ \\
\hline & $1 s 2 s-1 s 2 p$ & ${ }^{3} S-{ }^{3} P$ & 132.33 & $1-2$ & $6.977(9)$ & $3.053(-2)$ & $3.9898(-2)$ \\
\hline & & & 284.28 & $1-1$ & $5.614(8)$ & $6.802(-3)$ & $1.9097(-2)$ \\
\hline & & & 303.11 & $1-0$ & $5.646(8)$ & $2.592(-3)$ & $7.7604(-3)$ \\
\hline & & ${ }^{3} S-{ }^{1} P$ & 106.73 & $1-1$ & $2.443(9)$ & $4.173(-3)$ & $4.3988(-3)$ \\
\hline & & ${ }^{1} S-{ }^{1} P$ & 171.48 & $0-1$ & $2.656(9)$ & $1.171(-2)$ & $1.9833(-2)$ \\
\hline \multirow[t]{7}{*}{35} & $1 s^{2}-1 s 2 p$ & ${ }^{1} S-{ }^{3} P$ & 1.0087 & $0-1$ & $3.324(14)$ & $1.521(-1)$ & $5.0510(-4)$ \\
\hline & & ${ }^{1} S-{ }^{1} P$ & 1.0022 & $0-1$ & $1.360(15)$ & $6.143(-1)$ & $2.0268(-3)$ \\
\hline & $1 s 2 s-1 s 2 p$ & ${ }^{3} S-{ }^{3} P$ & 121.22 & $1-2$ & $8.543(9)$ & $3.137(-2)$ & $3.7552(-2)$ \\
\hline & & & 274.15 & $1-1$ & $5.807(8)$ & $6.543(-3)$ & $1.7717(-2)$ \\
\hline & & & 291.37 & $1-0$ & $5.972(8)$ & $2.534(-3)$ & $7.2910(-3)$ \\
\hline & & ${ }^{3} S-{ }^{1} P$ & 98.933 & $1-1$ & $3.048(9)$ & $4.473(-3)$ & $4.3707(-3)$ \\
\hline & & ${ }^{1} S-{ }^{1} P$ & 155.41 & $0-1$ & $3.316(9)$ & $1.201(-2)$ & $1.8427(-2)$ \\
\hline \multirow[t]{7}{*}{36} & $1 s^{2}-1 s 2 p$ & ${ }^{1} S-{ }^{3} P$ & .95181 & $0-1$ & $3.917(14)$ & $1.596(-1)$ & $5.0016(-4)$ \\
\hline & & ${ }^{1} S-{ }^{1} P$ & .94540 & $0-1$ & $1.506(15)$ & $6.056(-1)$ & $1.8848(-3)$ \\
\hline & $1 s 2 s-1 s 2 p$ & ${ }^{3} S-{ }^{3} P$ & 111.11 & $1-2$ & $1.046(10)$ & $3.226(-2)$ & $3.5403(-2)$ \\
\hline & & & 264.69 & $1-1$ & $5.999(8)$ & $6.301(-3)$ & $1.6472(-2)$ \\
\hline & & & 280.25 & $1-0$ & $6.316(8)$ & $2.479(-3)$ & $6.8611(-3)$ \\
\hline & & ${ }^{3} S-{ }^{1} P$ & 91.699 & $1-1$ & $3.789(9)$ & $4.776(-3)$ & $4.3255(-3)$ \\
\hline & & ${ }^{1} S-{ }^{1} P$ & 140.97 & $0-1$ & $4.137(9)$ & $1.233(-2)$ & $1.7160(-2)$ \\
\hline \multirow[t]{7}{*}{37} & $1 s^{2}-1 s 2 p$ & ${ }^{1} S-{ }^{3} P$ & .89955 & $0-1$ & $4.579(14)$ & $1.666(-1)$ & $4.9352(-4)$ \\
\hline & & ${ }^{1} S-{ }^{1} P$ & .89323 & $0-1$ & $1.664(15)$ & $5.972(-1)$ & $1.7561(-3)$ \\
\hline & $1 s 2 s-1 s 2 p$ & ${ }^{3} S-{ }^{3} P$ & 101.91 & $1-2$ & $1.280(10)$ & $3.321(-2)$ & $3.3429(-2)$ \\
\hline & & & 255.84 & $1-1$ & $6.190(8)$ & $6.074(-3)$ & $1.5347(-2)$ \\
\hline & & & 269.71 & $1-0$ & $6.678(8)$ & $2.427(-3)$ & $6.4662(-3)$ \\
\hline & & ${ }^{3} S-{ }^{1} P$ & 84.998 & $1-1$ & $4.692(9)$ & $5.082(-3)$ & $4.2663(-3)$ \\
\hline & & & & & & \multicolumn{2}{|c|}{ Continued on next page } \\
\hline
\end{tabular}


Table 15: E1 transitions

\begin{tabular}{|c|c|c|c|c|c|c|c|}
\hline \multicolumn{3}{|c|}{ Transition } & \multicolumn{4}{|c|}{$A_{k i}$} & \multirow[b]{2}{*}{ S (a.u.) } \\
\hline$Z$ & Array & Multiplet & $\lambda(\AA)$ & $J_{i}-J_{k}$ & $\left(s^{-1}\right)$ & $f_{i k}$ & \\
\hline \multirow{8}{*}{38} & & ${ }^{1} S-{ }^{1} P$ & 128.00 & $0-1$ & $5.158(9)$ & $1.267(-2)$ & $1.6015(-2)$ \\
\hline & $1 s^{2}-1 s 2 p$ & ${ }^{1} S-{ }^{3} P$ & .85141 & $0-1$ & $5.313(14)$ & $1.732(-1)$ & $4.8549(-4)$ \\
\hline & & ${ }^{1} S-{ }^{1} P$ & .84518 & $0-1$ & $1.834(15)$ & $5.892(-1)$ & $1.6394(-3)$ \\
\hline & $1 s 2 s-1 s 2 p$ & ${ }^{3} S-{ }^{3} P$ & 93.547 & $1-2$ & $1.565(10)$ & $3.422(-2)$ & $3.1612(-2)$ \\
\hline & & & 247.53 & $1-1$ & $6.380(8)$ & $5.861(-3)$ & $1.4327(-2)$ \\
\hline & & & 259.71 & $1-0$ & $7.059(8)$ & $2.379(-3)$ & $6.1027(-3)$ \\
\hline & & ${ }^{3} S-{ }^{1} P$ & 78.798 & $1-1$ & $5.791(9)$ & $5.391(-3)$ & $4.1954(-3)$ \\
\hline & & ${ }^{1} S-{ }^{1} P$ & 116.35 & $0-1$ & $6.422(9)$ & $1.303(-2)$ & $1.4977(-2)$ \\
\hline \multirow[t]{7}{*}{39} & $1 s^{2}-1 s 2 p$ & ${ }^{1} S-{ }^{3} P$ & .80697 & $0-1$ & $6.122(14)$ & $1.793(-1)$ & $4.7634(-4)$ \\
\hline & & ${ }^{1} S-{ }^{1} P$ & .80082 & $0-1$ & $2.016(15)$ & $5.815(-1)$ & $1.5332(-3)$ \\
\hline & $1 s 2 s-1 s 2 p$ & ${ }^{3} S-{ }^{3} P$ & 85.934 & $1-2$ & $1.912(10)$ & $3.527(-2)$ & $2.9936(-2)$ \\
\hline & & & 239.73 & $1-1$ & $6.570(8)$ & $5.660(-3)$ & $1.3401(-2)$ \\
\hline & & & 250.20 & $1-0$ & $7.460(8)$ & $2.334(-3)$ & $5.7674(-3)$ \\
\hline & & ${ }^{3} S-{ }^{1} P$ & 73.063 & $1-1$ & $7.126(9)$ & $5.703(-3)$ & $4.1154(-3)$ \\
\hline & & ${ }^{1} S-{ }^{1} P$ & 105.88 & $0-1$ & $7.985(9)$ & $1.342(-2)$ & $1.4035(-2)$ \\
\hline \multirow[t]{7}{*}{40} & $1 s^{2}-1 s 2 p$ & ${ }^{1} S-{ }^{3} P$ & .76586 & $0-1$ & 7.011(14) & $1.850(-1)$ & $4.6632(-4)$ \\
\hline & & ${ }^{1} S-{ }^{1} P$ & .75978 & $0-1$ & $2.212(15)$ & $5.742(-1)$ & $1.4363(-3)$ \\
\hline & $1 s 2 s-1 s 2 p$ & ${ }^{3} S-{ }^{3} P$ & 79.004 & $1-2$ & $2.333(10)$ & $3.638(-2)$ & $2.8386(-2)$ \\
\hline & & & 232.37 & $1-1$ & $6.760(8)$ & $5.472(-3)$ & $1.2558(-2)$ \\
\hline & & & 241.16 & $1-0$ & $7.884(8)$ & $2.291(-3)$ & $5.4573(-3)$ \\
\hline & & ${ }^{3} S-{ }^{1} P$ & 67.763 & $1-1$ & $8.743(9)$ & $6.019(-3)$ & $4.0281(-3)$ \\
\hline & & ${ }^{1} S-{ }^{1} P$ & 96.473 & $0-1$ & $9.912(9)$ & $1.383(-2)$ & $1.3178(-2)$ \\
\hline \multirow[t]{7}{*}{41} & $1 s^{2}-1 s 2 p$ & ${ }^{1} S-{ }^{3} P$ & .72777 & $0-1$ & $7.983(14)$ & $1.902(-1)$ & $4.5562(-4)$ \\
\hline & & ${ }^{1} S-{ }^{1} P$ & .72174 & $0-1$ & $2.421(15)$ & $5.673(-1)$ & $1.3479(-3)$ \\
\hline & $1 s 2 s-1 s 2 p$ & ${ }^{3} S-{ }^{3} P$ & 72.694 & $1-2$ & $2.843(10)$ & $3.754(-2)$ & $2.6950(-2)$ \\
\hline & & & 225.45 & $1-1$ & $6.948(8)$ & $5.295(-3)$ & $1.1789(-2)$ \\
\hline & & & 232.57 & $1-0$ & $8.328(8)$ & $2.251(-3)$ & $5.1701(-3)$ \\
\hline & & ${ }^{3} S-{ }^{1} P$ & 62.867 & $1-1$ & $1.070(10)$ & $6.338(-3)$ & $3.9354(-3)$ \\
\hline & & ${ }^{1} S-{ }^{1} P$ & 88.011 & $0-1$ & $1.228(10)$ & $1.426(-2)$ & $1.2396(-2)$ \\
\hline \multirow[t]{8}{*}{42} & $1 s^{2}-1 s 2 p$ & ${ }^{1} S-{ }^{3} P$ & .69239 & $0-1$ & $9.043(14)$ & $1.950(-1)$ & $4.4446(-4)$ \\
\hline & & ${ }^{1} S-{ }^{1} P$ & .68642 & $0-1$ & $2.645(15)$ & $5.606(-1)$ & $1.2668(-3)$ \\
\hline & $1 s 2 s-1 s 2 p$ & ${ }^{3} S-{ }^{3} P$ & 66.943 & $1-2$ & $3.460(10)$ & $3.875(-2)$ & $2.5617(-2)$ \\
\hline & & & 218.88 & $1-1$ & $7.140(8)$ & $5.128(-3)$ & $1.1086(-2)$ \\
\hline & & & 224.34 & $1-0$ & $8.799(8)$ & $2.213(-3)$ & $4.9035(-3)$ \\
\hline & & ${ }^{3} S-{ }^{1} P$ & 58.343 & $1-1$ & $1.306(10)$ & $6.662(-3)$ & $3.8389(-3)$ \\
\hline & & ${ }^{1} S-{ }^{1} P$ & 80.387 & $0-1$ & $1.519(10)$ & $1.471(-2)$ & $1.1681(-2)$ \\
\hline & & & & & & \multicolumn{2}{|c|}{ Continued on next page } \\
\hline
\end{tabular}


Table 15: E1 transitions

\begin{tabular}{|c|c|c|c|c|c|c|c|}
\hline \multicolumn{3}{|c|}{ Transition } & \multicolumn{4}{|c|}{$A_{k i}$} & \multirow[b]{2}{*}{$\mathrm{S}$ (a.u.) } \\
\hline$Z$ & Array & Multiplet & $\lambda(\AA)$ & $J_{i}-J_{k}$ & $\left(s^{-1}\right)$ & $f_{i k}$ & \\
\hline \multirow[t]{7}{*}{43} & \multirow{2}{*}{$1 s^{2}-1 s 2 p$} & ${ }^{1} S-{ }^{3} P$ & .65948 & $0-1$ & $1.020(15)$ & $1.994(-1)$ & $4.3297(-4)$ \\
\hline & & ${ }^{1} S-{ }^{1} P$ & .65355 & $0-1$ & $2.885(15)$ & $5.542(-1)$ & $1.1925(-3)$ \\
\hline & \multirow[t]{5}{*}{$1 s 2 s-1 s 2 p$} & ${ }^{3} S-{ }^{3} P$ & 61.701 & $1-2$ & $4.205(10)$ & $4.000(-2)$ & $2.4377(-2)$ \\
\hline & & & 212.67 & $1-1$ & $7.331(8)$ & $4.971(-3)$ & $1.0441(-2)$ \\
\hline & & & 216.51 & $1-0$ & $9.295(8)$ & $2.177(-3)$ & $4.6557(-3)$ \\
\hline & & ${ }^{3} S-{ }^{1} P$ & 54.167 & $1-1$ & $1.589(10)$ & $6.991(-3)$ & $3.7400(-3)$ \\
\hline & & ${ }^{1} S-{ }^{1} P$ & 73.517 & $0-1$ & $1.874(10)$ & $1.519(-2)$ & $1.1026(-2)$ \\
\hline \multirow[t]{7}{*}{44} & \multirow[t]{2}{*}{$1 s^{2}-1 s 2 p$} & ${ }^{1} S-{ }^{3} P$ & .62881 & $0-1$ & $1.144(15)$ & $2.035(-1)$ & $4.2128(-4)$ \\
\hline & & ${ }^{1} S-{ }^{1} P$ & .62292 & $0-1$ & $3.141(15)$ & $5.481(-1)$ & $1.1241(-3)$ \\
\hline & \multirow[t]{5}{*}{$1 s 2 s-1 s 2 p$} & ${ }^{3} S-{ }^{3} P$ & 56.918 & $1-2$ & $5.104(10)$ & $4.131(-2)$ & $2.3223(-2)$ \\
\hline & & & 206.76 & $1-1$ & $7.525(8)$ & $4.823(-3)$ & $9.8489(-3)$ \\
\hline & & & 209.01 & $1-0$ & $9.819(8)$ & $2.144(-3)$ & $4.4248(-3)$ \\
\hline & & ${ }^{3} S-{ }^{1} P$ & 50.311 & $1-1$ & $1.930(10)$ & $7.325(-3)$ & $3.6395(-3)$ \\
\hline & & ${ }^{1} S-{ }^{1} P$ & 67.318 & $0-1$ & $2.308(10)$ & $1.568(-2)$ & $1.0424(-2)$ \\
\hline \multirow[t]{7}{*}{45} & \multirow[t]{2}{*}{$1 s^{2}-1 s 2 p$} & ${ }^{1} S-{ }^{3} P$ & .60019 & $0-1$ & $1.279(15)$ & $2.072(-1)$ & $4.0950(-4)$ \\
\hline & & ${ }^{1} S-{ }^{1} P$ & .59433 & $0-1$ & $3.413(15)$ & $5.423(-1)$ & $1.0610(-3)$ \\
\hline & \multirow[t]{5}{*}{$1 s 2 s-1 s 2 p$} & ${ }^{3} S-{ }^{3} P$ & 52.552 & $1-2$ & $6.183(10)$ & $4.267(-2)$ & $2.2145(-2)$ \\
\hline & & & 201.16 & $1-1$ & $7.719(8)$ & $4.683(-3)$ & $9.3036(-3)$ \\
\hline & & & 201.84 & $1-0$ & $1.037(9)$ & $2.112(-3)$ & $4.2094(-3)$ \\
\hline & & ${ }^{3} S-{ }^{1} P$ & 46.751 & $1-1$ & $2.339(10)$ & $7.664(-3)$ & $3.5385(-3)$ \\
\hline & & ${ }^{1} S-{ }^{1} P$ & 61.720 & $0-1$ & $2.835(10)$ & $1.619(-2)$ & $9.8700(-3)$ \\
\hline \multirow[t]{7}{*}{46} & \multirow[t]{2}{*}{$1 s^{2}-1 s 2 p$} & ${ }^{1} S-{ }^{3} P$ & .57343 & $0-1$ & $1.425(15)$ & $2.107(-1)$ & $3.9771(-4)$ \\
\hline & & ${ }^{1} S-{ }^{1} P$ & .56760 & $0-1$ & $3.704(15)$ & $5.367(-1)$ & $1.0028(-3)$ \\
\hline & \multirow[t]{5}{*}{$1 s 2 s-1 s 2 p$} & ${ }^{3} S-{ }^{3} P$ & 48.564 & $1-2$ & $7.479(10)$ & $4.407(-2)$ & $2.1138(-2)$ \\
\hline & & & 195.83 & $1-1$ & $7.915(8)$ & $4.550(-3)$ & $8.8005(-3)$ \\
\hline & & & 194.99 & $1-0$ & $1.095(9)$ & $2.081(-3)$ & $4.0081(-3)$ \\
\hline & & ${ }^{3} S-{ }^{1} P$ & 43.465 & $1-1$ & $2.827(10)$ & $8.008(-3)$ & $3.4377(-3)$ \\
\hline & & ${ }^{1} S-{ }^{1} P$ & 56.658 & $0-1$ & $3.475(10)$ & $1.673(-2)$ & $9.3588(-3)$ \\
\hline \multirow[t]{7}{*}{47} & \multirow[t]{2}{*}{$1 s^{2}-1 s 2 p$} & ${ }^{1} S-{ }^{3} P$ & .54838 & $0-1$ & $1.581(15)$ & $2.138(-1)$ & $3.8600(-4)$ \\
\hline & & ${ }^{1} S-{ }^{1} P$ & .54258 & $0-1$ & $4.012(15)$ & $5.312(-1)$ & $9.4893(-4)$ \\
\hline & \multirow[t]{5}{*}{$1 s 2 s-1 s 2 p$} & ${ }^{3} S-{ }^{3} P$ & 44.919 & $1-2$ & $9.030(10)$ & $4.552(-2)$ & $2.0196(-2)$ \\
\hline & & & 190.74 & $1-1$ & $8.112(8)$ & $4.425(-3)$ & $8.3354(-3)$ \\
\hline & & & 188.42 & $1-0$ & $1.157(9)$ & $2.053(-3)$ & $3.8198(-3)$ \\
\hline & & ${ }^{3} S-{ }^{1} P$ & 40.430 & $1-1$ & $3.411(10)$ & $8.359(-3)$ & $3.3376(-3)$ \\
\hline & & ${ }^{1} S-{ }^{1} P$ & 52.075 & $0-1$ & $4.250(10)$ & $1.728(-2)$ & $8.8863(-3)$ \\
\hline
\end{tabular}


Table 15: E1 transitions

\begin{tabular}{|c|c|c|c|c|c|c|c|}
\hline \multicolumn{3}{|c|}{ Transition } & \multicolumn{4}{|c|}{$A_{k i}$} & \multirow[b]{2}{*}{ S (a.u.) } \\
\hline$Z$ & Array & Multiplet & $\lambda(\AA)$ & $J_{i}-J_{k}$ & $\left(s^{-1}\right)$ & $f_{i k}$ & \\
\hline \multirow[t]{7}{*}{48} & $1 s^{2}-1 s 2 p$ & ${ }^{1} S-{ }^{3} P$ & .52489 & $0-1$ & $1.749(15)$ & $2.167(-1)$ & $3.7442(-4)$ \\
\hline & & ${ }^{1} S-{ }^{1} P$ & .51911 & $0-1$ & $4.340(15)$ & $5.260(-1)$ & $8.9896(-4)$ \\
\hline & $1 s 2 s-1 s 2 p$ & ${ }^{3} S-{ }^{3} P$ & 41.584 & $1-2$ & $1.088(11)$ & $4.702(-2)$ & $1.9313(-2)$ \\
\hline & & & 185.90 & $1-1$ & $8.310(8)$ & $4.305(-3)$ & $7.9045(-3)$ \\
\hline & & & 182.13 & $1-0$ & $1.222(9)$ & $2.025(-3)$ & $3.6433(-3)$ \\
\hline & & ${ }^{3} S-{ }^{1} P$ & 37.629 & $1-1$ & $4.106(10)$ & $8.715(-3)$ & $3.2388(-3)$ \\
\hline & & ${ }^{1} S-{ }^{1} P$ & 47.921 & $0-1$ & $5.185(10)$ & $1.785(-2)$ & $8.4486(-3)$ \\
\hline \multirow[t]{7}{*}{49} & $1 s^{2}-1 s 2 p$ & ${ }^{1} S-{ }^{3} P$ & .50284 & $0-1$ & $1.928(15)$ & $2.193(-1)$ & $3.6302(-4)$ \\
\hline & & ${ }^{1} S-{ }^{1} P$ & .49708 & $0-1$ & $4.688(15)$ & $5.210(-1)$ & $8.5254(-4)$ \\
\hline & $1 s 2 s-1 s 2 p$ & ${ }^{3} S-{ }^{3} P$ & 38.530 & $1-2$ & $1.309(11)$ & $4.857(-2)$ & $1.8483(-2)$ \\
\hline & & & 181.27 & $1-1$ & $8.509(8)$ & $4.192(-3)$ & $7.5046(-3)$ \\
\hline & & & 176.10 & $1-0$ & $1.290(9)$ & $2.000(-3)$ & $3.4776(-3)$ \\
\hline & & ${ }^{3} S-{ }^{1} P$ & 35.040 & $1-1$ & $4.932(10)$ & $9.078(-3)$ & $3.1417(-3)$ \\
\hline & & ${ }^{1} S-{ }^{1} P$ & 44.152 & $0-1$ & $6.311(10)$ & $1.844(-2)$ & $8.0423(-3)$ \\
\hline \multirow[t]{7}{*}{50} & $1 s^{2}-1 s 2 p$ & ${ }^{1} S-{ }^{3} P$ & .48210 & $0-1$ & $2.120(15)$ & $2.216(-1)$ & $3.5177(-4)$ \\
\hline & & ${ }^{1} S-{ }^{1} P$ & .47637 & $0-1$ & $5.057(15)$ & $5.161(-1)$ & $8.0937(-4)$ \\
\hline & $1 s 2 s-1 s 2 p$ & ${ }^{3} S-{ }^{3} P$ & 35.731 & $1-2$ & $1.573(11)$ & $5.017(-2)$ & $1.7704(-2)$ \\
\hline & & & 176.82 & $1-1$ & $8.713(8)$ & $4.084(-3)$ & $7.1327(-3)$ \\
\hline & & & 170.29 & $1-0$ & $1.363(9)$ & $1.975(-3)$ & $3.3219(-3)$ \\
\hline & & ${ }^{3} S-{ }^{1} P$ & 32.648 & $1-1$ & $5.911(10)$ & $9.446(-3)$ & $3.0460(-3)$ \\
\hline & & ${ }^{1} S-{ }^{1} P$ & 40.726 & $0-1$ & $7.664(10)$ & $1.906(-2)$ & $7.6650(-3)$ \\
\hline \multirow[t]{7}{*}{51} & $1 s^{2}-1 s 2 p$ & ${ }^{1} S-{ }^{3} P$ & .46259 & $0-1$ & $2.325(15)$ & $2.238(-1)$ & $3.4082(-4)$ \\
\hline & & ${ }^{1} S-{ }^{1} P$ & .45687 & $0-1$ & $5.447(15)$ & $5.113(-1)$ & $7.6908(-4)$ \\
\hline & $1 s 2 s-1 s 2 p$ & ${ }^{3} S-{ }^{3} P$ & 33.166 & $1-2$ & $1.885(11)$ & $5.181(-2)$ & $1.6971(-2)$ \\
\hline & & & 172.59 & $1-1$ & $8.914(8)$ & $3.981(-3)$ & $6.7862(-3)$ \\
\hline & & & 164.75 & $1-0$ & $1.439(9)$ & $1.952(-3)$ & $3.1755(-3)$ \\
\hline & & ${ }^{3} S-{ }^{1} P$ & 30.438 & $1-1$ & $7.072(10)$ & $9.823(-3)$ & $2.9529(-3)$ \\
\hline & & ${ }^{1} S-{ }^{1} P$ & 37.610 & $0-1$ & $9.284(10)$ & $1.969(-2)$ & $7.3130(-3)$ \\
\hline \multirow[t]{7}{*}{52} & $1 s^{2}-1 s 2 p$ & ${ }^{1} S-{ }^{3} P$ & .44420 & $0-1$ & $2.544(15)$ & $2.258(-1)$ & $3.3014(-4)$ \\
\hline & & ${ }^{1} S-{ }^{1} P$ & .43850 & $0-1$ & $5.859(15)$ & $5.067(-1)$ & $7.3146(-4)$ \\
\hline & $1 s 2 s-1 s 2 p$ & ${ }^{3} S-{ }^{3} P$ & 30.810 & $1-2$ & $2.256(11)$ & $5.350(-2)$ & $1.6279(-2)$ \\
\hline & & & 168.52 & $1-1$ & $9.120(8)$ & $3.883(-3)$ & $6.4628(-3)$ \\
\hline & & & 159.40 & $1-0$ & $1.519(9)$ & $1.929(-3)$ & $3.0375(-3)$ \\
\hline & & ${ }^{3} S-{ }^{1} P$ & 28.394 & $1-1$ & $8.444(10)$ & $1.021(-2)$ & $2.8622(-3)$ \\
\hline & & ${ }^{1} S-{ }^{1} P$ & 34.771 & $0-1$ & $1.122(11)$ & $2.034(-2)$ & $6.9844(-3)$ \\
\hline \multirow[t]{2}{*}{53} & $1 s^{2}-1 s 2 p$ & ${ }^{1} S-{ }^{3} P$ & .42685 & $0-1$ & $2.776(15)$ & $2.275(-1)$ & $3.1973(-4)$ \\
\hline & & & & & & \multicolumn{2}{|c|}{ Continued on next page } \\
\hline
\end{tabular}


Table 15: E1 transitions

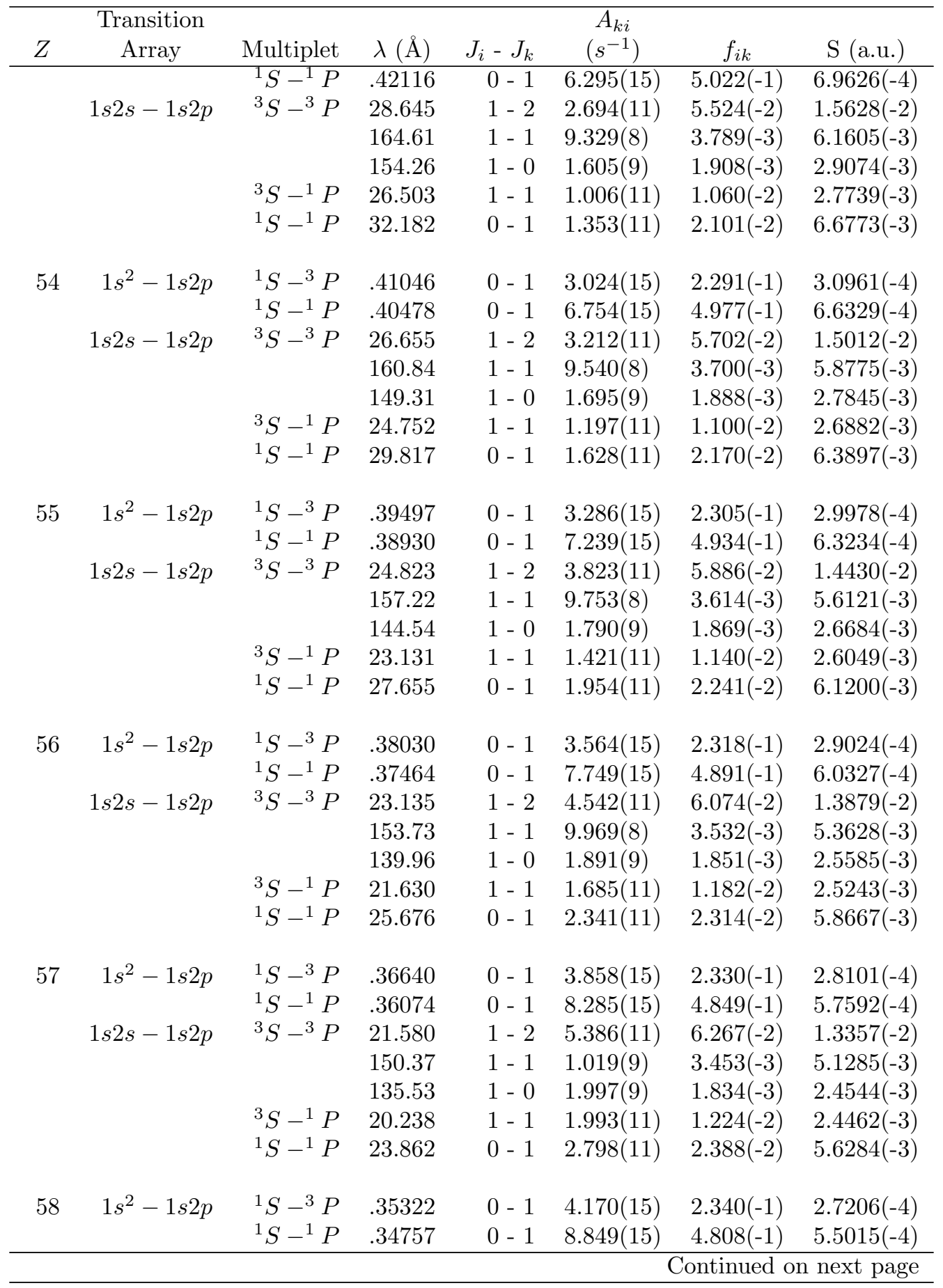


Table 15: E1 transitions

\begin{tabular}{|c|c|c|c|c|c|c|c|}
\hline \multicolumn{3}{|c|}{ Transition } & \multicolumn{4}{|c|}{$A_{k i}$} & \multirow[b]{2}{*}{ S (a.u.) } \\
\hline$Z$ & Array & Multiplet & $\lambda(\AA)$ & $J_{i}-J_{k}$ & $\left(s^{-1}\right)$ & $f_{i k}$ & \\
\hline & $1 s 2 s-1 s 2 p$ & ${ }^{3} S-{ }^{3} P$ & 20.144 & $1-2$ & $6.376(11)$ & $6.465(-2)$ & $1.2862(-2)$ \\
\hline & & & 147.14 & $1-1$ & $1.041(9)$ & $3.377(-3)$ & $4.9077(-3)$ \\
\hline & & & 131.28 & $1-0$ & $2.109(9)$ & $1.817(-3)$ & $2.3557(-3)$ \\
\hline & & ${ }^{3} S-{ }^{1} P$ & 18.947 & $1-1$ & $2.354(11)$ & $1.267(-2)$ & $2.3706(-3)$ \\
\hline & & ${ }^{1} S-{ }^{1} P$ & 22.197 & $0-1$ & $3.337(11)$ & $2.465(-2)$ & $5.4040(-3)$ \\
\hline \multirow[t]{7}{*}{59} & $1 s^{2}-1 s 2 p$ & ${ }^{1} S-{ }^{3} P$ & .34070 & $0-1$ & $4.498(15)$ & $2.348(-1)$ & $2.6341(-4)$ \\
\hline & & ${ }^{1} S-{ }^{1} P$ & .33506 & $0-1$ & $9.441(15)$ & $4.767(-1)$ & $5.2584(-4)$ \\
\hline & $1 s 2 s-1 s 2 p$ & ${ }^{3} S-{ }^{3} P$ & 18.819 & $1-2$ & $7.535(11)$ & $6.667(-2)$ & $1.2392(-2)$ \\
\hline & & & 144.00 & $1-1$ & $1.063(9)$ & $3.304(-3)$ & $4.6996(-3)$ \\
\hline & & & 127.18 & $1-0$ & $2.228(9)$ & $1.801(-3)$ & $2.2621(-3)$ \\
\hline & & ${ }^{3} S-{ }^{1} P$ & 17.748 & $1-1$ & $2.775(11)$ & $1.311(-2)$ & $2.2975(-3)$ \\
\hline & & ${ }^{1} S-{ }^{1} P$ & 20.668 & $0-1$ & $3.972(11)$ & $2.544(-2)$ & $5.1924(-3)$ \\
\hline \multirow[t]{7}{*}{60} & $1 s^{2}-1 s 2 p$ & ${ }^{1} S-{ }^{3} P$ & .32881 & $0-1$ & $4.845(15)$ & $2.356(-1)$ & $2.5505(-4)$ \\
\hline & & ${ }^{1} S-{ }^{1} P$ & .32318 & $0-1$ & $1.006(16)$ & $4.727(-1)$ & $5.0289(-4)$ \\
\hline & $1 s 2 s-1 s 2 p$ & ${ }^{3} S-{ }^{3} P$ & 17.593 & $1-2$ & $8.889(11)$ & $6.875(-2)$ & $1.1945(-2)$ \\
\hline & & & 141.00 & $1-1$ & $1.085(9)$ & $3.234(-3)$ & $4.5031(-3)$ \\
\hline & & & 123.23 & $1-0$ & $2.353(9)$ & $1.786(-3)$ & $2.1732(-3)$ \\
\hline & & ${ }^{3} S-{ }^{1} P$ & 16.636 & $1-1$ & $3.267(11)$ & $1.355(-2)$ & $2.2269(-3)$ \\
\hline & & ${ }^{1} S-{ }^{1} P$ & 19.262 & $0-1$ & $4.718(11)$ & $2.624(-2)$ & $4.9925(-3)$ \\
\hline \multirow[t]{7}{*}{61} & $1 s^{2}-1 s 2 p$ & ${ }^{1} S-{ }^{3} P$ & .31751 & $0-1$ & $5.211(15)$ & $2.363(-1)$ & $2.4696(-4)$ \\
\hline & & ${ }^{1} S-{ }^{1} P$ & .31188 & $0-1$ & $1.071(16)$ & $4.687(-1)$ & $4.8118(-4)$ \\
\hline & $1 s 2 s-1 s 2 p$ & ${ }^{3} S-{ }^{3} P$ & 16.461 & $1-2$ & $1.047(12)$ & $7.086(-2)$ & $1.1520(-2)$ \\
\hline & & & 138.17 & $1-1$ & $1.105(9)$ & $3.164(-3)$ & $4.3173(-3)$ \\
\hline & & & 119.48 & $1-0$ & $2.481(9)$ & $1.770(-3)$ & $2.0886(-3)$ \\
\hline & & ${ }^{3} S-{ }^{1} P$ & 15.603 & $1-1$ & $3.838(11)$ & $1.401(-2)$ & $2.1587(-3)$ \\
\hline & & ${ }^{1} S-{ }^{1} P$ & 17.969 & $0-1$ & $5.591(11)$ & $2.707(-2)$ & $4.8035(-3)$ \\
\hline \multirow[t]{7}{*}{62} & $1 s^{2}-1 s 2 p$ & ${ }^{1} S-{ }^{3} P$ & .30674 & $0-1$ & $5.596(15)$ & $2.368(-1)$ & $2.3915(-4)$ \\
\hline & & ${ }^{1} S-{ }^{1} P$ & .30112 & $0-1$ & $1.139(16)$ & $4.647(-1)$ & $4.6064(-4)$ \\
\hline & $1 s 2 s-1 s 2 p$ & ${ }^{3} S-{ }^{3} P$ & 15.412 & $1-2$ & $1.231(12)$ & $7.303(-2)$ & $1.1116(-2)$ \\
\hline & & & 135.31 & $1-1$ & $1.129(9)$ & $3.099(-3)$ & $4.1415(-3)$ \\
\hline & & & 115.76 & $1-0$ & $2.623(9)$ & $1.756(-3)$ & $2.0082(-3)$ \\
\hline & & ${ }^{3} S-{ }^{1} P$ & 14.642 & $1-1$ & $4.503(11)$ & $1.447(-2)$ & $2.0929(-3)$ \\
\hline & & ${ }^{1} S-{ }^{1} P$ & 16.776 & $0-1$ & $6.616(11)$ & $2.791(-2)$ & $4.6246(-3)$ \\
\hline \multirow[t]{4}{*}{63} & $1 s^{2}-1 s 2 p$ & ${ }^{1} S-{ }^{3} P$ & .29649 & $0-1$ & $6.001(15)$ & $2.373(-1)$ & $2.3160(-4)$ \\
\hline & & ${ }^{1} S-{ }^{1} P$ & .29087 & $0-1$ & $1.211(16)$ & $4.607(-1)$ & $4.4117(-4)$ \\
\hline & $1 s 2 s-1 s 2 p$ & ${ }^{3} S-{ }^{3} P$ & 14.438 & $1-2$ & $1.445(12)$ & $7.525(-2)$ & $1.0731(-2)$ \\
\hline & & & & & & \multicolumn{2}{|c|}{ Continued on next page } \\
\hline
\end{tabular}


Table 15: E1 transitions

\begin{tabular}{|c|c|c|c|c|c|c|c|}
\hline \multicolumn{3}{|c|}{ Transition } & \multicolumn{4}{|c|}{$A_{k i}$} & \multirow[b]{2}{*}{ S (a.u.) } \\
\hline$Z$ & Array & Multiplet & $\lambda(\AA)$ & $J_{i}-J_{k}$ & $\left(s^{-1}\right)$ & $f_{i k}$ & \\
\hline \multirow{11}{*}{64} & & & 132.63 & $1-1$ & $1.151(9)$ & $3.035(-3)$ & $3.9750(-3)$ \\
\hline & & & 112.24 & $1-0$ & $2.768(9)$ & $1.743(-3)$ & $1.9316(-3)$ \\
\hline & & ${ }^{3} S-{ }^{1} P$ & 13.748 & $1-1$ & $5.274(11)$ & $1.495(-2)$ & $2.0293(-3)$ \\
\hline & & ${ }^{1} S-{ }^{1} P$ & 15.675 & $0-1$ & $7.812(11)$ & $2.878(-2)$ & $4.4551(-3)$ \\
\hline & $1 s^{2}-1 s 2 p$ & ${ }^{1} S-{ }^{3} P$ & .28671 & $0-1$ & $6.428(15)$ & $2.376(-1)$ & $2.2431(-4)$ \\
\hline & & ${ }^{1} S-{ }^{1} P$ & .28109 & $0-1$ & $1.285(16)$ & $4.568(-1)$ & $4.2270(-4)$ \\
\hline & $1 s 2 s-1 s 2 p$ & ${ }^{3} S-{ }^{3} P$ & 13.536 & $1-2$ & $1.693(12)$ & $7.752(-2)$ & $1.0364(-2)$ \\
\hline & & & 129.97 & $1-1$ & $1.174(9)$ & $2.974(-3)$ & $3.8171(-3)$ \\
\hline & & & 108.79 & $1-0$ & $2.925(9)$ & $1.730(-3)$ & $1.8586(-3)$ \\
\hline & & ${ }^{3} S-{ }^{1} P$ & 12.916 & $1-1$ & $6.169(11)$ & $1.543(-2)$ & $1.9679(-3)$ \\
\hline & & ${ }^{1} S-{ }^{1} P$ & 14.658 & $0-1$ & $9.208(11)$ & $2.966(-2)$ & $4.2942(-3)$ \\
\hline \multirow[t]{7}{*}{65} & $1 s^{2}-1 s 2 p$ & ${ }^{1} S-{ }^{3} P$ & .27739 & $0-1$ & $6.875(15)$ & $2.379(-1)$ & $2.1727(-4)$ \\
\hline & & ${ }^{1} S-{ }^{1} P$ & .27177 & $0-1$ & $1.363(16)$ & $4.529(-1)$ & $4.0517(-4)$ \\
\hline & $1 s 2 s-1 s 2 p$ & ${ }^{3} S-{ }^{3} P$ & 12.698 & $1-2$ & $1.982(12)$ & $7.984(-2)$ & $1.0013(-2)$ \\
\hline & & & 127.40 & $1-1$ & $1.198(9)$ & $2.914(-3)$ & $3.6671(-3)$ \\
\hline & & & 105.47 & $1-0$ & $3.090(9)$ & $1.718(-3)$ & $1.7890(-3)$ \\
\hline & & ${ }^{3} S-{ }^{1} P$ & 12.140 & $1-1$ & $7.204(11)$ & $1.592(-2)$ & $1.9086(-3)$ \\
\hline & & ${ }^{1} S-{ }^{1} P$ & 13.718 & $0-1$ & $1.083(12)$ & $3.057(-2)$ & $4.1415(-3)$ \\
\hline \multirow[t]{7}{*}{66} & $1 s^{2}-1 s 2 p$ & ${ }^{1} S-{ }^{3} P$ & .26848 & $0-1$ & $7.345(15)$ & $2.381(-1)$ & $2.1047(-4)$ \\
\hline & & ${ }^{1} S-{ }^{1} P$ & .26287 & $0-1$ & $1.445(16)$ & $4.489(-1)$ & $3.8851(-4)$ \\
\hline & $1 s 2 s-1 s 2 p$ & ${ }^{3} S-{ }^{3} P$ & 11.920 & $1-2$ & $2.316(12)$ & $8.221(-2)$ & $9.6788(-3)$ \\
\hline & & & 124.93 & $1-1$ & $1.221(9)$ & $2.857(-3)$ & $3.5246(-3)$ \\
\hline & & & 102.27 & $1-0$ & $3.263(9)$ & $1.706(-3)$ & $1.7226(-3)$ \\
\hline & & ${ }^{3} S-{ }^{1} P$ & 11.418 & $1-1$ & $8.401(11)$ & $1.642(-2)$ & $1.8514(-3)$ \\
\hline & & ${ }^{1} S-{ }^{1} P$ & 12.848 & $0-1$ & $1.273(12)$ & $3.149(-2)$ & $3.9962(-3)$ \\
\hline \multirow[t]{7}{*}{67} & $1 s^{2}-1 s 2 p$ & ${ }^{1} S-{ }^{3} P$ & .25998 & $0-1$ & $7.838(15)$ & $2.383(-1)$ & $2.0392(-4)$ \\
\hline & & ${ }^{1} S-{ }^{1} P$ & .25436 & $0-1$ & $1.529(16)$ & $4.450(-1)$ & $3.7267(-4)$ \\
\hline & $1 s 2 s-1 s 2 p$ & ${ }^{3} S-{ }^{3} P$ & 11.197 & $1-2$ & $2.702(12)$ & $8.463(-2)$ & $9.3591(-3)$ \\
\hline & & & 122.54 & $1-1$ & $1.244(9)$ & $2.800(-3)$ & $3.3890(-3)$ \\
\hline & & & 99.174 & $1-0$ & $3.446(9)$ & $1.694(-3)$ & $1.6592(-3)$ \\
\hline & & ${ }^{3} S-{ }^{1} P$ & 10.744 & $1-1$ & $9.782(11)$ & $1.693(-2)$ & $1.7962(-3)$ \\
\hline & & ${ }^{1} S-{ }^{1} P$ & 12.042 & $0-1$ & $1.492(12)$ & $3.244(-2)$ & $3.8580(-3)$ \\
\hline \multirow[t]{5}{*}{68} & $1 s^{2}-1 s 2 p$ & ${ }^{1} S-{ }^{3} P$ & .25184 & $0-1$ & $8.354(15)$ & $2.383(-1)$ & $1.9757(-4)$ \\
\hline & & ${ }^{1} S-{ }^{1} P$ & .24623 & $0-1$ & $1.618(16)$ & $4.411(-1)$ & $3.5757(-4)$ \\
\hline & $1 s 2 s-1 s 2 p$ & ${ }^{3} S-{ }^{3} P$ & 10.524 & $1-2$ & $3.147(12)$ & $8.710(-2)$ & $9.0533(-3)$ \\
\hline & & & 120.24 & $1-1$ & $1.267(9)$ & $2.745(-3)$ & $3.2600(-3)$ \\
\hline & & & & & & \multicolumn{2}{|c|}{ Continued on next page } \\
\hline
\end{tabular}


Table 15: E1 transitions

\begin{tabular}{|c|c|c|c|c|c|c|c|}
\hline \multicolumn{3}{|c|}{ Transition } & \multicolumn{4}{|c|}{$A_{k i}$} & \multirow[b]{2}{*}{ S (a.u.) } \\
\hline$Z$ & Array & Multiplet & $\lambda(\AA)$ & $J_{i}-J_{k}$ & $\left(s^{-1}\right)$ & $f_{i k}$ & \\
\hline \multirow{10}{*}{69} & & & 96.200 & $1-0$ & $3.638(9)$ & $1.683(-3)$ & $1.5986(-3)$ \\
\hline & & ${ }^{3} S-{ }^{1} P$ & 10.115 & $1-1$ & $1.137(12)$ & $1.745(-2)$ & $1.7429(-3)$ \\
\hline & & ${ }^{1} S-{ }^{1} P$ & 11.295 & $0-1$ & $1.747(12)$ & $3.340(-2)$ & $3.7263(-3)$ \\
\hline & \multirow[t]{2}{*}{$1 s^{2}-1 s 2 p$} & ${ }^{1} S-{ }^{3} P$ & .24406 & $0-1$ & $8.895(15)$ & $2.383(-1)$ & $1.9146(-4)$ \\
\hline & & ${ }^{1} S-{ }^{1} P$ & .23845 & $0-1$ & $1.710(16)$ & $4.372(-1)$ & $3.4320(-4)$ \\
\hline & \multirow[t]{5}{*}{$1 s 2 s-1 s 2 p$} & ${ }^{3} S-{ }^{3} P$ & 9.8976 & $1-2$ & $3.661(12)$ & $8.962(-2)$ & $8.7607(-3)$ \\
\hline & & & 118.02 & $1-1$ & $1.289(9)$ & $2.691(-3)$ & $3.1370(-3)$ \\
\hline & & & 93.333 & $1-0$ & $3.839(9)$ & $1.671(-3)$ & $1.5406(-3)$ \\
\hline & & ${ }^{3} S-{ }^{1} P$ & 9.5283 & $1-1$ & $1.321(12)$ & $1.797(-2)$ & $1.6914(-3)$ \\
\hline & & ${ }^{1} S-{ }^{1} P$ & 10.602 & $0-1$ & $2.041(12)$ & $3.439(-2)$ & $3.6008(-3)$ \\
\hline \multirow[t]{7}{*}{70} & \multirow[t]{2}{*}{$1 s^{2}-1 s 2 p$} & ${ }^{1} S-{ }^{3} P$ & .23661 & $0-1$ & $9.460(15)$ & $2.382(-1)$ & $1.8555(-4)$ \\
\hline & & ${ }^{1} S-{ }^{1} P$ & .23099 & $0-1$ & $1.805(16)$ & $4.333(-1)$ & $3.2950(-4)$ \\
\hline & \multirow[t]{5}{*}{$1 s 2 s-1 s 2 p$} & ${ }^{3} S-{ }^{3} P$ & 9.3141 & $1-2$ & $4.253(12)$ & $9.219(-2)$ & $8.4804(-3)$ \\
\hline & & & 115.90 & $1-1$ & $1.310(9)$ & $2.638(-3)$ & $3.0197(-3)$ \\
\hline & & & 90.582 & $1-0$ & $4.049(9)$ & $1.660(-3)$ & $1.4851(-3)$ \\
\hline & & ${ }^{3} S-{ }^{1} P$ & 8.9801 & $1-1$ & $1.531(12)$ & $1.851(-2)$ & $1.6417(-3)$ \\
\hline & & ${ }^{1} S-{ }^{1} P$ & 9.9578 & $0-1$ & $2.381(12)$ & $3.539(-2)$ & $3.4810(-3)$ \\
\hline \multirow[t]{7}{*}{71} & \multirow[t]{2}{*}{$1 s^{2}-1 s 2 p$} & ${ }^{1} S-{ }^{3} P$ & .22947 & $0-1$ & $1.005(16)$ & $2.381(-1)$ & $1.7984(-4)$ \\
\hline & & ${ }^{1} S-{ }^{1} P$ & .22385 & $0-1$ & $1.905(16)$ & $4.294(-1)$ & $3.1641(-4)$ \\
\hline & \multirow[t]{5}{*}{$1 s 2 s-1 s 2 p$} & ${ }^{3} S-{ }^{3} P$ & 8.7696 & $1-2$ & $4.934(12)$ & $9.481(-2)$ & $8.2119(-3)$ \\
\hline & & & 113.81 & $1-1$ & $1.332(9)$ & $2.587(-3)$ & $2.9077(-3)$ \\
\hline & & & 87.892 & $1-0$ & $4.273(9)$ & $1.650(-3)$ & $1.4320(-3)$ \\
\hline & & ${ }^{3} S-{ }^{1} P$ & 8.4673 & $1-1$ & $1.773(12)$ & $1.906(-2)$ & $1.5937(-3)$ \\
\hline & & ${ }^{1} S-{ }^{1} P$ & 9.3588 & $0-1$ & $2.774(12)$ & $3.642(-2)$ & $3.3665(-3)$ \\
\hline \multirow[t]{7}{*}{72} & \multirow[t]{2}{*}{$1 s^{2}-1 s 2 p$} & ${ }^{1} S-{ }^{3} P$ & .22263 & $0-1$ & $1.067(16)$ & $2.379(-1)$ & $1.7433(-4)$ \\
\hline & & ${ }^{1} S-{ }^{1} P$ & .21701 & $0-1$ & $2.009(16)$ & $4.254(-1)$ & $3.0393(-4)$ \\
\hline & \multirow[t]{5}{*}{$1 s 2 s-1 s 2 p$} & ${ }^{3} S-{ }^{3} P$ & 8.2617 & $1-2$ & $5.716(12)$ & $9.749(-2)$ & $7.9543(-3)$ \\
\hline & & & 111.80 & $1-1$ & $1.354(9)$ & $2.536(-3)$ & $2.8008(-3)$ \\
\hline & & & 85.308 & $1-0$ & $4.507(9)$ & $1.639(-3)$ & $1.3810(-3)$ \\
\hline & & ${ }^{3} S-{ }^{1} P$ & 7.9878 & $1-1$ & $2.051(12)$ & $1.961(-2)$ & $1.5474(-3)$ \\
\hline & & ${ }^{1} S-{ }^{1} P$ & 8.8015 & $0-1$ & $3.226(12)$ & $3.747(-2)$ & $3.2572(-3)$ \\
\hline \multirow[t]{6}{*}{73} & \multirow[t]{2}{*}{$1 s^{2}-1 s 2 p$} & ${ }^{1} S-{ }^{3} P$ & .21606 & $0-1$ & $1.132(16)$ & $2.376(-1)$ & $1.6900(-4)$ \\
\hline & & ${ }^{1} S-{ }^{1} P$ & .21044 & $0-1$ & $2.116(16)$ & $4.215(-1)$ & $2.9199(-4)$ \\
\hline & \multirow[t]{4}{*}{$1 s 2 s-1 s 2 p$} & ${ }^{3} S-{ }^{3} P$ & 7.7872 & $1-2$ & $6.614(12)$ & $1.002(-1)$ & $7.7071(-3)$ \\
\hline & & & 109.86 & $1-1$ & $1.375(9)$ & $2.487(-3)$ & $2.6985(-3)$ \\
\hline & & & 82.808 & $1-0$ & $4.754(9)$ & $1.629(-3)$ & $1.3322(-3)$ \\
\hline & & & & & & \multicolumn{2}{|c|}{ Continued on next page } \\
\hline
\end{tabular}


Table 15: E1 transitions

\begin{tabular}{|c|c|c|c|c|c|c|c|}
\hline \multicolumn{3}{|c|}{ Transition } & \multicolumn{4}{|c|}{$A_{k i}$} & \multirow[b]{2}{*}{ S (a.u.) } \\
\hline$Z$ & Array & Multiplet & $\lambda(\AA)$ & $J_{i}-J_{k}$ & $\left(s^{-1}\right)$ & $f_{i k}$ & \\
\hline \multirow{9}{*}{74} & & ${ }^{3} S-{ }^{1} P$ & 7.5389 & $1-1$ & $2.369(12)$ & $2.018(-2)$ & $1.5026(-3)$ \\
\hline & & ${ }^{1} S-{ }^{1} P$ & 8.2825 & $0-1$ & $3.747(12)$ & $3.854(-2)$ & $3.1525(-3)$ \\
\hline & $1 s^{2}-1 s 2 p$ & ${ }^{1} S-{ }^{3} P$ & .20976 & $0-1$ & $1.199(16)$ & $2.373(-1)$ & $1.6386(-4)$ \\
\hline & & ${ }^{1} S-{ }^{1} P$ & .20414 & $0-1$ & $2.227(16)$ & $4.175(-1)$ & $2.8058(-4)$ \\
\hline & $1 s 2 s-1 s 2 p$ & ${ }^{3} S-{ }^{3} P$ & 7.3439 & $1-2$ & $7.642(12)$ & $1.030(-1)$ & $7.4698(-3)$ \\
\hline & & & 107.99 & $1-1$ & $1.395(9)$ & $2.438(-3)$ & $2.6007(-3)$ \\
\hline & & & 80.399 & $1-0$ & $5.011(9)$ & $1.619(-3)$ & $1.2854(-3)$ \\
\hline & & ${ }^{3} S-{ }^{1} P$ & 7.1185 & $1-1$ & $2.732(12)$ & $2.076(-2)$ & $1.4593(-3)$ \\
\hline & & ${ }^{1} S-{ }^{1} P$ & 7.7988 & $0-1$ & $4.346(12)$ & $3.963(-2)$ & $3.0523(-3)$ \\
\hline \multirow[t]{7}{*}{75} & $1 s^{2}-1 s 2 p$ & ${ }^{1} S-{ }^{3} P$ & .20371 & $0-1$ & $1.269(16)$ & $2.369(-1)$ & $1.5888(-4)$ \\
\hline & & ${ }^{1} S-{ }^{1} P$ & .19809 & $0-1$ & $2.343(16)$ & $4.135(-1)$ & $2.6966(-4)$ \\
\hline & $1 s 2 s-1 s 2 p$ & ${ }^{3} S-{ }^{3} P$ & 6.9291 & $1-2$ & $8.821(12)$ & $1.058(-1)$ & $7.2418(-3)$ \\
\hline & & & 106.15 & $1-1$ & $1.416(9)$ & $2.391(-3)$ & $2.5070(-3)$ \\
\hline & & & 78.047 & $1-0$ & $5.287(9)$ & $1.609(-3)$ & $1.2404(-3)$ \\
\hline & & ${ }^{3} S-{ }^{1} P$ & 6.7243 & $1-1$ & $3.149(12)$ & $2.134(-2)$ & $1.4175(-3)$ \\
\hline & & ${ }^{1} S-{ }^{1} P$ & 7.3472 & $0-1$ & $5.034(12)$ & $4.074(-2)$ & $2.9563(-3)$ \\
\hline \multirow[t]{7}{*}{76} & $1 s^{2}-1 s 2 p$ & ${ }^{1} S-{ }^{3} P$ & .19789 & $0-1$ & $1.343(16)$ & $2.365(-1)$ & $1.5407(-4)$ \\
\hline & & ${ }^{1} S-{ }^{1} P$ & .19227 & $0-1$ & $2.463(16)$ & $4.095(-1)$ & $2.5920(-4)$ \\
\hline & $1 s 2 s-1 s 2 p$ & ${ }^{3} S-{ }^{3} P$ & 6.5410 & $1-2$ & $1.017(13)$ & $1.087(-1)$ & $7.0226(-3)$ \\
\hline & & & 104.38 & $1-1$ & $1.435(9)$ & $2.345(-3)$ & $2.4173(-3)$ \\
\hline & & & 75.783 & $1-0$ & $5.574(9)$ & $1.600(-3)$ & $1.1973(-3)$ \\
\hline & & ${ }^{3} S-{ }^{1} P$ & 6.3548 & $1-1$ & $3.624(12)$ & $2.194(-2)$ & $1.3771(-3)$ \\
\hline & & ${ }^{1} S-{ }^{1} P$ & 6.9258 & $0-1$ & $5.823(12)$ & $4.187(-2)$ & $2.8642(-3)$ \\
\hline \multirow[t]{7}{*}{77} & $1 s^{2}-1 s 2 p$ & ${ }^{1} S-{ }^{3} P$ & .19230 & $0-1$ & $1.419(16)$ & $2.360(-1)$ & $1.4942(-4)$ \\
\hline & & ${ }^{1} S-{ }^{1} P$ & .18667 & $0-1$ & $2.587(16)$ & $4.055(-1)$ & $2.4918(-4)$ \\
\hline & $1 s 2 s-1 s 2 p$ & ${ }^{3} S-{ }^{3} P$ & 6.1775 & $1-2$ & $1.171(13)$ & $1.116(-1)$ & $6.8118(-3)$ \\
\hline & & & 102.68 & $1-1$ & $1.454(9)$ & $2.299(-3)$ & $2.3313(-3)$ \\
\hline & & & 73.596 & $1-0$ & $5.875(9)$ & $1.590(-3)$ & $1.1558(-3)$ \\
\hline & & ${ }^{3} S-{ }^{1} P$ & 6.0082 & $1-1$ & $4.167(12)$ & $2.255(-2)$ & $1.3380(-3)$ \\
\hline & & ${ }^{1} S-{ }^{1} P$ & 6.5320 & $0-1$ & $6.727(12)$ & $4.303(-2)$ & $2.7759(-3)$ \\
\hline \multirow[t]{7}{*}{78} & $1 s^{2}-1 s 2 p$ & ${ }^{1} S-{ }^{3} P$ & .18692 & $0-1$ & $1.499(16)$ & $2.355(-1)$ & $1.4492(-4)$ \\
\hline & & ${ }^{1} S-{ }^{1} P$ & .18129 & $0-1$ & $2.715(16)$ & $4.014(-1)$ & $2.3958(-4)$ \\
\hline & $1 s 2 s-1 s 2 p$ & ${ }^{3} S-{ }^{3} P$ & 5.8370 & $1-2$ & $1.347(13)$ & $1.146(-1)$ & $6.6088(-3)$ \\
\hline & & & 101.06 & $1-1$ & $1.471(9)$ & $2.253(-3)$ & $2.2488(-3)$ \\
\hline & & & 71.494 & $1-0$ & $6.187(9)$ & $1.580(-3)$ & $1.1159(-3)$ \\
\hline & & ${ }^{3} S-{ }^{1} P$ & 5.6828 & $1-1$ & $4.785(12)$ & $2.317(-2)$ & $1.3002(-3)$ \\
\hline & & & & & & \multicolumn{2}{|c|}{ Continued on next page } \\
\hline
\end{tabular}


Table 15: E1 transitions

\begin{tabular}{|c|c|c|c|c|c|c|c|}
\hline \multicolumn{3}{|c|}{ Transition } & \multicolumn{4}{|c|}{$A_{k i}$} & \multirow[b]{2}{*}{ S (a.u.) } \\
\hline$Z$ & Array & Multiplet & $\lambda(\AA)$ & $J_{i}-J_{k}$ & $\left(s^{-1}\right)$ & $f_{i k}$ & \\
\hline & & ${ }^{1} S-{ }^{1} P$ & 6.1639 & $0-1$ & $7.761(12)$ & $4.420(-2)$ & $2.6911(-3)$ \\
\hline \multirow[t]{7}{*}{79} & $1 s^{2}-1 s 2 p$ & ${ }^{1} S-{ }^{3} P$ & .18174 & $0-1$ & $1.581(16)$ & $2.349(-1)$ & $1.4057(-4)$ \\
\hline & & ${ }^{1} S-{ }^{1} P$ & .17611 & $0-1$ & $2.848(16)$ & $3.973(-1)$ & $2.3037(-4)$ \\
\hline & $1 s 2 s-1 s 2 p$ & ${ }^{3} S-{ }^{3} P$ & 5.5177 & $1-2$ & $1.547(13)$ & $1.177(-1)$ & $6.4133(-3)$ \\
\hline & & & 99.493 & $1-1$ & $1.488(9)$ & $2.208(-3)$ & $2.1696(-3)$ \\
\hline & & & 69.458 & $1-0$ & $6.515(9)$ & $1.571(-3)$ & $1.0776(-3)$ \\
\hline & & ${ }^{3} S-{ }^{1} P$ & 5.3772 & $1-1$ & $5.489(12)$ & $2.379(-2)$ & $1.2636(-3)$ \\
\hline & & ${ }^{1} S-{ }^{1} P$ & 5.8194 & $0-1$ & $8.943(12)$ & $4.540(-2)$ & $2.6095(-3)$ \\
\hline \multirow[t]{7}{*}{80} & $1 s^{2}-1 s 2 p$ & ${ }^{1} S-{ }^{3} P$ & .17676 & $0-1$ & $1.668(16)$ & $2.343(-1)$ & $1.3636(-4)$ \\
\hline & & ${ }^{1} S-{ }^{1} P$ & .17112 & $0-1$ & $2.986(16)$ & $3.932(-1)$ & $2.2153(-4)$ \\
\hline & $1 s 2 s-1 s 2 p$ & ${ }^{3} S-{ }^{3} P$ & 5.2182 & $1-2$ & $1.775(13)$ & $1.208(-1)$ & $6.2250(-3)$ \\
\hline & & & 98.029 & $1-1$ & $1.501(9)$ & $2.162(-3)$ & $2.0935(-3)$ \\
\hline & & & 67.511 & $1-0$ & $6.852(9)$ & $1.561(-3)$ & $1.0406(-3)$ \\
\hline & & ${ }^{3} S-{ }^{1} P$ & 5.0901 & $1-1$ & $6.290(12)$ & $2.443(-2)$ & $1.2282(-3)$ \\
\hline & & ${ }^{1} S-{ }^{1} P$ & 5.4970 & $0-1$ & $1.029(13)$ & $4.662(-2)$ & $2.5311(-3)$ \\
\hline \multirow[t]{7}{*}{81} & $1 s^{2}-1 s 2 p$ & ${ }^{1} S-{ }^{3} P$ & .17195 & $0-1$ & $1.757(16)$ & $2.337(-1)$ & $1.3229(-4)$ \\
\hline & & ${ }^{1} S-{ }^{1} P$ & .16632 & $0-1$ & $3.128(16)$ & $3.891(-1)$ & $2.1304(-4)$ \\
\hline & $1 s 2 s-1 s 2 p$ & ${ }^{3} S-{ }^{3} P$ & 4.9368 & $1-2$ & $2.035(13)$ & $1.239(-1)$ & $6.0434(-3)$ \\
\hline & & & 96.554 & $1-1$ & $1.516(9)$ & $2.119(-3)$ & $2.0204(-3)$ \\
\hline & & & 65.597 & $1-0$ & $7.215(9)$ & $1.551(-3)$ & $1.0051(-3)$ \\
\hline & & ${ }^{3} S-{ }^{1} P$ & 4.8199 & $1-1$ & $7.202(12)$ & $2.508(-2)$ & $1.1940(-3)$ \\
\hline & & ${ }^{1} S-{ }^{1} P$ & 5.1946 & $0-1$ & $1.183(13)$ & $4.787(-2)$ & $2.4557(-3)$ \\
\hline \multirow[t]{7}{*}{82} & $1 s^{2}-1 s 2 p$ & ${ }^{1} S-{ }^{3} P$ & .16732 & $0-1$ & $1.850(16)$ & $2.330(-1)$ & $1.2834(-4)$ \\
\hline & & ${ }^{1} S-{ }^{1} P$ & .16168 & $0-1$ & $3.274(16)$ & $3.849(-1)$ & $2.0489(-4)$ \\
\hline & $1 s 2 s-1 s 2 p$ & ${ }^{3} S-{ }^{3} P$ & 4.6715 & $1-2$ & $2.333(13)$ & $1.272(-1)$ & $5.8682(-3)$ \\
\hline & & & 94.713 & $1-1$ & $1.550(9)$ & $2.085(-3)$ & $1.9501(-3)$ \\
\hline & & & 63.553 & $1-0$ & $7.663(9)$ & $1.547(-3)$ & $9.7084(-4)$ \\
\hline & & ${ }^{3} S-{ }^{1} P$ & 4.5647 & $1-1$ & $8.242(12)$ & $2.575(-2)$ & $1.1608(-3)$ \\
\hline & & ${ }^{1} S-{ }^{1} P$ & 4.9113 & $0-1$ & $1.359(13)$ & $4.913(-2)$ & $2.3831(-3)$ \\
\hline \multirow[t]{8}{*}{83} & $1 s^{2}-1 s 2 p$ & ${ }^{1} S-{ }^{3} P$ & .16286 & $0-1$ & $1.947(16)$ & $2.323(-1)$ & $1.2452(-4)$ \\
\hline & & ${ }^{1} S-{ }^{1} P$ & .15721 & $0-1$ & $3.425(16)$ & $3.807(-1)$ & $1.9706(-4)$ \\
\hline & $1 s 2 s-1 s 2 p$ & ${ }^{3} S-{ }^{3} P$ & 4.4243 & $1-2$ & $2.667(13)$ & $1.304(-1)$ & $5.6992(-3)$ \\
\hline & & & 93.893 & $1-1$ & $1.536(9)$ & $2.030(-3)$ & $1.8824(-3)$ \\
\hline & & & 62.005 & $1-0$ & $7.971(9)$ & $1.531(-3)$ & $9.3783(-4)$ \\
\hline & & ${ }^{3} S-{ }^{1} P$ & 4.3267 & $1-1$ & $9.411(12)$ & $2.641(-2)$ & $1.1286(-3)$ \\
\hline & & ${ }^{1} S-{ }^{1} P$ & 4.6453 & $0-1$ & $1.559(13)$ & $5.042(-2)$ & $2.3131(-3)$ \\
\hline & & & & & & \multicolumn{2}{|c|}{ Continued on next page } \\
\hline
\end{tabular}


Table 15: E1 transitions

\begin{tabular}{|c|c|c|c|c|c|c|c|}
\hline \multicolumn{3}{|c|}{ Transition } & \multicolumn{4}{|c|}{$A_{k i}$} & \multirow[b]{2}{*}{$\mathrm{S}$ (a.u.) } \\
\hline$Z$ & Array & Multiplet & $\lambda(\AA)$ & $J_{i}-J_{k}$ & $\left(s^{-1}\right)$ & $f_{i k}$ & \\
\hline \multirow[t]{7}{*}{84} & \multirow{2}{*}{$1 s^{2}-1 s 2 p$} & ${ }^{1} S-{ }^{3} P$ & .15855 & $0-1$ & $2.047(16)$ & $2.315(-1)$ & $1.2082(-4)$ \\
\hline & & ${ }^{1} S-{ }^{1} P$ & .15290 & $0-1$ & $3.581(16)$ & $3.765(-1)$ & $1.8952(-4)$ \\
\hline & \multirow[t]{5}{*}{$1 s 2 s-1 s 2 p$} & ${ }^{3} S-{ }^{3} P$ & 4.1908 & $1-2$ & $3.048(13)$ & $1.338(-1)$ & $5.5359(-3)$ \\
\hline & & & 92.701 & $1-1$ & $1.541(9)$ & $1.985(-3)$ & $1.8173(-3)$ \\
\hline & & & 60.318 & $1-0$ & $8.365(9)$ & $1.521(-3)$ & $9.0601(-4)$ \\
\hline & & ${ }^{3} S-{ }^{1} P$ & 4.1016 & $1-1$ & $1.074(13)$ & $2.709(-2)$ & $1.0975(-3)$ \\
\hline & & ${ }^{1} S-{ }^{1} P$ & 4.3956 & $0-1$ & $1.786(13)$ & $5.173(-2)$ & $2.2457(-3)$ \\
\hline \multirow[t]{7}{*}{85} & \multirow[t]{2}{*}{$1 s^{2}-1 s 2 p$} & ${ }^{1} S-{ }^{3} P$ & .15439 & $0-1$ & $2.151(16)$ & $2.307(-1)$ & $1.1723(-4)$ \\
\hline & & ${ }^{1} S-{ }^{1} P$ & .14874 & $0-1$ & $3.741(16)$ & $3.723(-1)$ & $1.8228(-4)$ \\
\hline & \multirow[t]{5}{*}{$1 s 2 s-1 s 2 p$} & ${ }^{3} S-{ }^{3} P$ & 3.9711 & $1-2$ & $3.480(13)$ & $1.371(-1)$ & $5.3782(-3)$ \\
\hline & & & 91.597 & $1-1$ & $1.542(9)$ & $1.939(-3)$ & $1.7546(-3)$ \\
\hline & & & 58.698 & $1-0$ & $8.769(9)$ & $1.510(-3)$ & $8.7531(-4)$ \\
\hline & & ${ }^{3} S-{ }^{1} P$ & 3.8894 & $1-1$ & $1.225(13)$ & $2.778(-2)$ & $1.0673(-3)$ \\
\hline & & ${ }^{1} S-{ }^{1} P$ & 4.1612 & $0-1$ & $2.044(13)$ & $5.306(-2)$ & $2.1806(-3)$ \\
\hline \multirow[t]{7}{*}{86} & \multirow[t]{2}{*}{$1 s^{2}-1 s 2 p$} & ${ }^{1} S-{ }^{3} P$ & .15038 & $0-1$ & $2.259(16)$ & $2.298(-1)$ & $1.1376(-4)$ \\
\hline & & ${ }^{1} S-{ }^{1} P$ & .14472 & $0-1$ & $3.907(16)$ & $3.680(-1)$ & $1.7531(-4)$ \\
\hline & \multirow[t]{5}{*}{$1 s 2 s-1 s 2 p$} & ${ }^{3} S-{ }^{3} P$ & 3.7644 & $1-2$ & $3.970(13)$ & $1.406(-1)$ & $5.2257(-3)$ \\
\hline & & & 90.664 & $1-1$ & $1.535(9)$ & $1.892(-3)$ & $1.6941(-3)$ \\
\hline & & & 57.175 & $1-0$ & $9.168(9)$ & $1.498(-3)$ & $8.4569(-4)$ \\
\hline & & ${ }^{3} S-{ }^{1} P$ & 3.6896 & $1-1$ & $1.396(13)$ & $2.849(-2)$ & $1.0380(-3)$ \\
\hline & & ${ }^{1} S-{ }^{1} P$ & 3.9409 & $0-1$ & $2.337(13)$ & $5.441(-2)$ & $2.1178(-3)$ \\
\hline \multirow[t]{7}{*}{87} & \multirow[t]{2}{*}{$1 s^{2}-1 s 2 p$} & ${ }^{1} S-{ }^{3} P$ & .14649 & $0-1$ & $2.372(16)$ & $2.289(-1)$ & $1.1039(-4)$ \\
\hline & & ${ }^{1} S-{ }^{1} P$ & .14083 & $0-1$ & $4.077(16)$ & $3.637(-1)$ & $1.6860(-4)$ \\
\hline & \multirow[t]{5}{*}{$1 s 2 s-1 s 2 p$} & ${ }^{3} S-{ }^{3} P$ & 3.5693 & $1-2$ & $4.525(13)$ & $1.441(-1)$ & $5.0782(-3)$ \\
\hline & & & 89.657 & $1-1$ & $1.533(9)$ & $1.847(-3)$ & $1.6358(-3)$ \\
\hline & & & 55.649 & $1-0$ & $9.607(9)$ & $1.487(-3)$ & $8.1709(-4)$ \\
\hline & & ${ }^{3} S-{ }^{1} P$ & 3.5008 & $1-1$ & $1.589(13)$ & $2.920(-2)$ & $1.0096(-3)$ \\
\hline & & ${ }^{1} S-{ }^{1} P$ & 3.7334 & $0-1$ & $2.670(13)$ & $5.579(-2)$ & $2.0572(-3)$ \\
\hline \multirow[t]{7}{*}{88} & \multirow[t]{2}{*}{$1 s^{2}-1 s 2 p$} & ${ }^{1} S-{ }^{3} P$ & .14274 & $0-1$ & $2.488(16)$ & $2.280(-1)$ & $1.0712(-4)$ \\
\hline & & ${ }^{1} S-{ }^{1} P$ & .13707 & $0-1$ & $4.252(16)$ & $3.593(-1)$ & $1.6214(-4)$ \\
\hline & \multirow[t]{5}{*}{$1 s 2 s-1 s 2 p$} & ${ }^{3} S-{ }^{3} P$ & 3.3856 & $1-2$ & $5.154(13)$ & $1.476(-1)$ & $4.9355(-3)$ \\
\hline & & & 88.824 & $1-1$ & $1.522(9)$ & $1.801(-3)$ & $1.5796(-3)$ \\
\hline & & & 54.214 & $1-0$ & $1.004(10)$ & $1.474(-3)$ & $7.8946(-4)$ \\
\hline & & ${ }^{3} S-{ }^{1} P$ & 3.3229 & $1-1$ & $1.808(13)$ & $2.992(-2)$ & $9.8205(-4)$ \\
\hline & & ${ }^{1} S-{ }^{1} P$ & 3.5382 & $0-1$ & $3.047(13)$ & $5.719(-2)$ & $1.9986(-3)$ \\
\hline
\end{tabular}


Table 15: E1 transitions

\begin{tabular}{|c|c|c|c|c|c|c|c|}
\hline \multicolumn{3}{|c|}{ Transition } & \multicolumn{4}{|c|}{$A_{k i}$} & \multirow[b]{2}{*}{ S (a.u.) } \\
\hline$Z$ & Array & Multiplet & $\lambda(\AA)$ & $J_{i}-J_{k}$ & $\left(s^{-1}\right)$ & $f_{i k}$ & \\
\hline \multirow[t]{7}{*}{89} & $1 s^{2}-1 s 2 p$ & ${ }^{1} S-{ }^{3} P$ & .13911 & $0-1$ & $2.608(16)$ & $2.270(-1)$ & $1.0395(-4)$ \\
\hline & & ${ }^{1} S-{ }^{1} P$ & .13344 & $0-1$ & $4.432(16)$ & $3.549(-1)$ & $1.5591(-4)$ \\
\hline & $1 s 2 s-1 s 2 p$ & ${ }^{3} S-{ }^{3} P$ & 3.2124 & $1-2$ & $5.864(13)$ & $1.512(-1)$ & $4.7973(-3)$ \\
\hline & & & 88.089 & $1-1$ & $1.507(9)$ & $1.753(-3)$ & $1.5254(-3)$ \\
\hline & & & 52.837 & $1-0$ & $1.048(10)$ & $1.462(-3)$ & $7.6277(-4)$ \\
\hline & & ${ }^{3} S-{ }^{1} P$ & 3.1548 & $1-1$ & $2.055(13)$ & $3.066(-2)$ & $9.5530(-4)$ \\
\hline & & ${ }^{1} S-{ }^{1} P$ & 3.3544 & $0-1$ & $3.475(13)$ & $5.862(-2)$ & $1.9419(-3)$ \\
\hline \multirow[t]{7}{*}{90} & $1 s^{2}-1 s 2 p$ & ${ }^{1} S-{ }^{3} P$ & .13560 & $0-1$ & $2.732(16)$ & $2.260(-1)$ & $1.0088(-4)$ \\
\hline & & ${ }^{1} S-{ }^{1} P$ & .12993 & $0-1$ & $4.616(16)$ & $3.505(-1)$ & $1.4991(-4)$ \\
\hline & $1 s 2 s-1 s 2 p$ & ${ }^{3} S-{ }^{3} P$ & 3.0489 & $1-2$ & $6.668(13)$ & $1.549(-1)$ & $4.6635(-3)$ \\
\hline & & & 87.437 & $1-1$ & $1.488(9)$ & $1.706(-3)$ & $1.4730(-3)$ \\
\hline & & & 51.510 & $1-0$ & $1.093(10)$ & $1.449(-3)$ & $7.3697(-4)$ \\
\hline & & ${ }^{3} S-{ }^{1} P$ & 2.9961 & $1-1$ & $2.334(13)$ & $3.141(-2)$ & $9.2933(-4)$ \\
\hline & & ${ }^{1} S-{ }^{1} P$ & 3.1812 & $0-1$ & $3.959(13)$ & $6.006(-2)$ & $1.8871(-3)$ \\
\hline \multirow[t]{7}{*}{91} & $1 s^{2}-1 s 2 p$ & ${ }^{1} S-{ }^{3} P$ & .13221 & $0-1$ & $2.861(16)$ & $2.249(-1)$ & $9.7889(-5)$ \\
\hline & & ${ }^{1} S-{ }^{1} P$ & .12652 & $0-1$ & $4.806(16)$ & $3.460(-1)$ & $1.4412(-4)$ \\
\hline & $1 s 2 s-1 s 2 p$ & ${ }^{3} S-{ }^{3} P$ & 2.8946 & $1-2$ & $7.575(13)$ & $1.586(-1)$ & $4.5339(-3)$ \\
\hline & & & 86.947 & $1-1$ & $1.462(9)$ & $1.656(-3)$ & $1.4224(-3)$ \\
\hline & & & 50.253 & $1-0$ & $1.137(10)$ & $1.435(-3)$ & $7.1202(-4)$ \\
\hline & & ${ }^{3} S-{ }^{1} P$ & 2.8461 & $1-1$ & $2.649(13)$ & $3.216(-2)$ & $9.0411(-4)$ \\
\hline & & ${ }^{1} S-{ }^{1} P$ & 3.0179 & $0-1$ & $4.507(13)$ & $6.153(-2)$ & $1.8341(-3)$ \\
\hline \multirow[t]{7}{*}{92} & $1 s^{2}-1 s 2 p$ & ${ }^{1} S-{ }^{3} P$ & .12892 & $0-1$ & $2.994(16)$ & $2.238(-1)$ & $9.4994(-5)$ \\
\hline & & ${ }^{1} S-{ }^{1} P$ & .12323 & $0-1$ & $5.001(16)$ & $3.415(-1)$ & $1.3855(-4)$ \\
\hline & $1 s 2 s-1 s 2 p$ & ${ }^{3} S-{ }^{3} P$ & 2.7488 & $1-2$ & $8.600(13)$ & $1.624(-1)$ & $4.4082(-3)$ \\
\hline & & & 86.565 & $1-1$ & $1.430(9)$ & $1.607(-3)$ & $1.3735(-3)$ \\
\hline & & & 49.047 & $1-0$ & $1.181(10)$ & $1.420(-3)$ & $6.8789(-4)$ \\
\hline & & ${ }^{3} S-{ }^{1} P$ & 2.7043 & $1-1$ & $3.004(13)$ & $3.293(-2)$ & $8.7960(-4)$ \\
\hline & & ${ }^{1} S-{ }^{1} P$ & 2.8638 & $0-1$ & $5.126(13)$ & $6.303(-2)$ & $1.7827(-3)$ \\
\hline \multirow[t]{7}{*}{93} & $1 s^{2}-1 s 2 p$ & ${ }^{1} S-{ }^{3} P$ & .12573 & $0-1$ & $3.132(16)$ & $2.227(-1)$ & $9.2176(-5)$ \\
\hline & & ${ }^{1} S-{ }^{1} P$ & .12004 & $0-1$ & $5.200(16)$ & $3.370(-1)$ & $1.3316(-4)$ \\
\hline & $1 s 2 s-1 s 2 p$ & ${ }^{3} S-{ }^{3} P$ & 2.6111 & $1-2$ & $9.757(13)$ & $1.662(-1)$ & $4.2862(-3)$ \\
\hline & & & 86.331 & $1-1$ & $1.392(9)$ & $1.556(-3)$ & $1.3263(-3)$ \\
\hline & & & 47.899 & $1-0$ & $1.225(10)$ & $1.405(-3)$ & $6.6453(-4)$ \\
\hline & & ${ }^{3} S-{ }^{1} P$ & 2.5702 & $1-1$ & $3.404(13)$ & $3.371(-2)$ & $8.5577(-4)$ \\
\hline & & ${ }^{1} S-{ }^{1} P$ & 2.7184 & $0-1$ & $5.826(13)$ & $6.455(-2)$ & $1.7330(-3)$ \\
\hline \multirow[t]{2}{*}{94} & $1 s^{2}-1 s 2 p$ & ${ }^{1} S-{ }^{3} P$ & .12265 & $0-1$ & $3.274(16)$ & $2.215(-1)$ & $8.9444(-5)$ \\
\hline & & & & & & \multicolumn{2}{|c|}{ Continued on next page } \\
\hline
\end{tabular}


Table 15: E1 transitions

\begin{tabular}{|c|c|c|c|c|c|c|c|}
\hline \multicolumn{3}{|c|}{ Transition } & \multicolumn{4}{|c|}{$A_{k i}$} & \multirow[b]{2}{*}{ S (a.u.) } \\
\hline$Z$ & Array & Multiplet & $\lambda(\AA)$ & $J_{i}-J_{k}$ & $\left(s^{-1}\right)$ & $f_{i k}$ & \\
\hline & & ${ }^{1} S-{ }^{1} P$ & .11694 & $0-1$ & $5.405(16)$ & $3.324(-1)$ & $1.2798(-4)$ \\
\hline & $1 s 2 s-1 s 2 p$ & ${ }^{3} S-{ }^{3} P$ & 2.4809 & $1-2$ & $1.106(14)$ & $1.701(-1)$ & $4.1679(-3)$ \\
\hline & & & 86.327 & $1-1$ & $1.344(9)$ & $1.502(-3)$ & $1.2806(-3)$ \\
\hline & & & 46.827 & $1-0$ & $1.267(10)$ & $1.388(-3)$ & $6.4192(-4)$ \\
\hline & & ${ }^{3} S-{ }^{1} P$ & 2.4433 & $1-1$ & $3.855(13)$ & $3.450(-2)$ & $8.3261(-4)$ \\
\hline & & ${ }^{1} S-{ }^{1} P$ & 2.5812 & $0-1$ & $6.616(13)$ & $6.609(-2)$ & $1.6847(-3)$ \\
\hline \multirow[t]{7}{*}{95} & $1 s^{2}-1 s 2 p$ & ${ }^{1} S-{ }^{3} P$ & .11965 & $0-1$ & $3.422(16)$ & $2.203(-1)$ & $8.6783(-5)$ \\
\hline & & ${ }^{1} S-{ }^{1} P$ & .11394 & $0-1$ & $5.614(16)$ & $3.278(-1)$ & $1.2296(-4)$ \\
\hline & $1 s 2 s-1 s 2 p$ & ${ }^{3} S-{ }^{3} P$ & 2.3577 & $1-2$ & $1.253(14)$ & $1.741(-1)$ & $4.0530(-3)$ \\
\hline & & & 86.407 & $1-1$ & $1.294(9)$ & $1.449(-3)$ & $1.2364(-3)$ \\
\hline & & & 45.783 & $1-0$ & $1.309(10)$ & $1.371(-3)$ & $6.2002(-4)$ \\
\hline & & ${ }^{3} S-{ }^{1} P$ & 2.3231 & $1-1$ & $4.364(13)$ & $3.531(-2)$ & $8.1007(-4)$ \\
\hline & & ${ }^{1} S-{ }^{1} P$ & 2.4514 & $0-1$ & $7.509(13)$ & $6.765(-2)$ & $1.6379(-3)$ \\
\hline \multirow[t]{7}{*}{96} & $1 s^{2}-1 s 2 p$ & ${ }^{1} S-{ }^{3} P$ & .11675 & $0-1$ & $3.573(16)$ & $2.191(-1)$ & $8.4201(-5)$ \\
\hline & & ${ }^{1} S-{ }^{1} P$ & .11103 & $0-1$ & $5.828(16)$ & $3.232(-1)$ & $1.1812(-4)$ \\
\hline & $1 s 2 s-1 s 2 p$ & ${ }^{3} S-{ }^{3} P$ & 2.2411 & $1-2$ & $1.419(14)$ & $1.781(-1)$ & $3.9414(-3)$ \\
\hline & & & 86.782 & $1-1$ & $1.233(9)$ & $1.393(-3)$ & $1.1936(-3)$ \\
\hline & & & 44.819 & $1-0$ & $1.348(10)$ & $1.353(-3)$ & $5.9881(-4)$ \\
\hline & & ${ }^{3} S-{ }^{1} P$ & 2.2093 & $1-1$ & $4.936(13)$ & $3.612(-2)$ & $7.8815(-4)$ \\
\hline & & ${ }^{1} S-{ }^{1} P$ & 2.3288 & $0-1$ & $8.516(13)$ & $6.924(-2)$ & $1.5925(-3)$ \\
\hline \multirow[t]{7}{*}{97} & $1 s^{2}-1 s 2 p$ & ${ }^{1} S-{ }^{3} P$ & .11393 & $0-1$ & $3.730(16)$ & $2.178(-1)$ & $8.1686(-5)$ \\
\hline & & ${ }^{1} S-{ }^{1} P$ & .10821 & $0-1$ & $6.047(16)$ & $3.185(-1)$ & $1.1345(-4)$ \\
\hline & $1 s 2 s-1 s 2 p$ & ${ }^{3} S-{ }^{3} P$ & 2.1308 & $1-2$ & $1.605(14)$ & $1.821(-1)$ & $3.8329(-3)$ \\
\hline & & & 87.441 & $1-1$ & $1.164(9)$ & $1.334(-3)$ & $1.1521(-3)$ \\
\hline & & & 43.923 & $1-0$ & $1.383(10)$ & $1.333(-3)$ & $5.7825(-4)$ \\
\hline & & ${ }^{3} S-{ }^{1} P$ & 2.1016 & $1-1$ & $5.580(13)$ & $3.694(-2)$ & $7.6680(-4)$ \\
\hline & & ${ }^{1} S-{ }^{1} P$ & 2.2129 & $0-1$ & $9.651(13)$ & $7.085(-2)$ & $1.5484(-3)$ \\
\hline \multirow[t]{7}{*}{98} & $1 s^{2}-1 s 2 p$ & ${ }^{1} S-{ }^{3} P$ & .11121 & $0-1$ & $3.892(16)$ & $2.165(-1)$ & $7.9244(-5)$ \\
\hline & & ${ }^{1} S-{ }^{1} P$ & .10547 & $0-1$ & $6.271(16)$ & $3.137(-1)$ & $1.0894(-4)$ \\
\hline & $1 s 2 s-1 s 2 p$ & ${ }^{3} S-{ }^{3} P$ & 2.0263 & $1-2$ & $1.815(14)$ & $1.863(-1)$ & $3.7274(-3)$ \\
\hline & & & 88.394 & $1-1$ & $1.087(9)$ & $1.274(-3)$ & $1.1120(-3)$ \\
\hline & & & 43.082 & $1-0$ & $1.415(10)$ & $1.312(-3)$ & $5.5832(-4)$ \\
\hline & & ${ }^{3} S-{ }^{1} P$ & 1.9994 & $1-1$ & $6.304(13)$ & $3.778(-2)$ & $7.4602(-4)$ \\
\hline & & ${ }^{1} S-{ }^{1} P$ & 2.1032 & $0-1$ & $1.093(14)$ & $7.248(-2)$ & $1.5056(-3)$ \\
\hline \multirow[t]{3}{*}{99} & $1 s^{2}-1 s 2 p$ & ${ }^{1} S-{ }^{3} P$ & .10855 & $0-1$ & $4.059(16)$ & $2.151(-1)$ & $7.6867(-5)$ \\
\hline & & ${ }^{1} S-{ }^{1} P$ & .10281 & $0-1$ & $6.500(16)$ & $3.090(-1)$ & $1.0458(-4)$ \\
\hline & & & & & & \multicolumn{2}{|c|}{ Continued on next page } \\
\hline
\end{tabular}


Table 15: E1 transitions

\begin{tabular}{|c|c|c|c|c|c|c|c|}
\hline \multicolumn{3}{|c|}{ Transition } & \multicolumn{4}{|c|}{$A_{k i}$} & \multirow[b]{2}{*}{ S (a.u.) } \\
\hline$Z$ & Array & Multiplet & $\lambda(\AA)$ & $J_{i}-J_{k}$ & $\left(s^{-1}\right)$ & $f_{i k}$ & \\
\hline \multirow{12}{*}{100} & $1 s 2 s-1 s 2 p$ & ${ }^{3} S-{ }^{3} P$ & 1.9274 & $1-2$ & $2.052(14)$ & $1.904(-1)$ & $3.6249(-3)$ \\
\hline & & & 89.866 & $1-1$ & $9.986(8)$ & $1.209(-3)$ & $1.0731(-3)$ \\
\hline & & & 42.345 & $1-0$ & $1.438(10)$ & $1.289(-3)$ & $5.3900(-4)$ \\
\hline & & ${ }^{3} S-{ }^{1} P$ & 1.9026 & $1-1$ & $7.117(13)$ & $3.862(-2)$ & $7.2577(-4)$ \\
\hline & & ${ }^{1} S-{ }^{1} P$ & 1.9994 & $0-1$ & $1.237(14)$ & $7.414(-2)$ & $1.4640(-3)$ \\
\hline & $1 s^{2}-1 s 2 p$ & ${ }^{1} S-{ }^{3} P$ & .10597 & $0-1$ & $4.231(16)$ & $2.137(-1)$ & $7.4554(-5)$ \\
\hline & & ${ }^{1} S-{ }^{1} P$ & .10022 & $0-1$ & $6.733(16)$ & $3.042(-1)$ & $1.0037(-4)$ \\
\hline & $1 s 2 s-1 s 2 p$ & ${ }^{3} S-{ }^{3} P$ & 1.8336 & $1-2$ & $2.317(14)$ & $1.947(-1)$ & $3.5251(-3)$ \\
\hline & & & 91.813 & $1-1$ & $9.035(8)$ & $1.142(-3)$ & $1.0354(-3)$ \\
\hline & & & 41.673 & $1-0$ & $1.457(10)$ & $1.264(-3)$ & $5.2026(-4)$ \\
\hline & & ${ }^{3} S-{ }^{1} P$ & 1.8108 & $1-1$ & $8.031(13)$ & $3.948(-2)$ & $7.0604(-4)$ \\
\hline & & ${ }^{1} S-{ }^{1} P$ & 1.9011 & $0-1$ & $1.399(14)$ & $7.582(-2)$ & $1.4235(-3)$ \\
\hline
\end{tabular}


Table 16: M1 and M2 transitions

\begin{tabular}{|c|c|c|c|c|c|c|c|}
\hline \multicolumn{3}{|c|}{ Transition } & \multicolumn{5}{|c|}{$A_{k i}$} \\
\hline$Z$ & Array & Multiplet & $\lambda(\AA)$ & $J_{i}-J_{k}$ & $\left(s^{-1}\right)$ & Туре & S (a.u.) \\
\hline \multirow[t]{2}{*}{2} & $1 s^{2}-1 s 2 s$ & ${ }^{1} S-{ }^{3} S$ & 625.48 & $0-1$ & $1.266(-4)$ & M1 & $3.4468(-9)$ \\
\hline & $1 s^{2}-1 s 2 p$ & ${ }^{1} S-{ }^{3} P$ & 591.33 & $0-2$ & $3.271(-1)$ & M2 & 7.9312 \\
\hline \multirow[t]{2}{*}{3} & $1 s^{2}-1 s 2 s$ & ${ }^{1} S-{ }^{3} S$ & 210.05 & $0-1$ & $2.035(-2)$ & M1 & $2.0980(-8)$ \\
\hline & $1 s^{2}-1 s 2 p$ & ${ }^{1} S-{ }^{3} P$ & 202.30 & $0-2$ & $3.498(1)$ & M2 & 3.9755 \\
\hline \multirow[t]{2}{*}{4} & $1 s^{2}-1 s 2 s$ & ${ }^{1} S-{ }^{3} S$ & 104.54 & $0-1$ & $5.615(-1)$ & M1 & $7.1343(-8)$ \\
\hline & $1 s^{2}-1 s 2 p$ & ${ }^{1} S-{ }^{3} P$ & 101.68 & $0-2$ & $6.171(2)$ & M2 & 2.2497 \\
\hline \multirow[t]{2}{*}{5} & $1 s^{2}-1 s 2 s$ & ${ }^{1} S-{ }^{3} S$ & 62.436 & $0-1$ & 6.696 & M1 & $1.8127(-7)$ \\
\hline & $1 s^{2}-1 s 2 p$ & ${ }^{1} S-{ }^{3} P$ & 61.085 & $0-2$ & $5.014(3)$ & M2 & 1.4299 \\
\hline \multirow[t]{2}{*}{6} & $1 s^{2}-1 s 2 s$ & ${ }^{1} S-{ }^{3} S$ & 41.470 & $0-1$ & $4.860(1)$ & M1 & $3.8550(-7)$ \\
\hline & $1 s^{2}-1 s 2 p$ & ${ }^{1} S-{ }^{3} P$ & 40.726 & $0-2$ & $2.622(4)$ & M2 & $9.8495(-1)$ \\
\hline \multirow[t]{2}{*}{7} & $1 s^{2}-1 s 2 s$ & ${ }^{1} S-{ }^{3} S$ & 29.533 & $0-1$ & $2.537(2)$ & M1 & $7.2683(-7)$ \\
\hline & $1 s^{2}-1 s 2 p$ & ${ }^{1} S-{ }^{3} P$ & 29.081 & $0-2$ & $1.030(5)$ & M2 & $7.1824(-1)$ \\
\hline \multirow[t]{2}{*}{8} & $1 s^{2}-1 s 2 s$ & ${ }^{1} S-{ }^{3} S$ & 22.097 & $0-1$ & $1.047(3)$ & M1 & $1.2561(-6)$ \\
\hline & $1 s^{2}-1 s 2 p$ & ${ }^{1} S-{ }^{3} P$ & 21.800 & $0-2$ & $3.308(5)$ & M2 & $5.4628(-1)$ \\
\hline \multirow[t]{2}{*}{9} & $1 s^{2}-1 s 2 s$ & ${ }^{1} S-{ }^{3} S$ & 17.152 & $0-1$ & $3.621(3)$ & M1 & $2.0323(-6)$ \\
\hline & $1 s^{2}-1 s 2 p$ & ${ }^{1} S-{ }^{3} P$ & 16.947 & $0-2$ & $9.155(5)$ & M2 & $4.2912(-1)$ \\
\hline \multirow[t]{2}{*}{10} & $1 s^{2}-1 s 2 s$ & ${ }^{1} S-{ }^{3} S$ & 13.699 & $0-1$ & $1.092(4)$ & M1 & $3.1226(-6)$ \\
\hline & $1 s^{2}-1 s 2 p$ & ${ }^{1} S-{ }^{3} P$ & 13.550 & $0-2$ & $2.257(6)$ & M2 & $3.4577(-1)$ \\
\hline \multirow[t]{2}{*}{11} & $1 s^{2}-1 s 2 s$ & ${ }^{1} S-{ }^{3} S$ & 11.191 & $0-1$ & $2.952(4)$ & M1 & $4.6022(-6)$ \\
\hline & $1 s^{2}-1 s 2 p$ & ${ }^{1} S-{ }^{3} P$ & 11.080 & $0-2$ & $5.079(6)$ & M2 & $2.8440(-1)$ \\
\hline \multirow[t]{2}{*}{12} & $1 s^{2}-1 s 2 s$ & ${ }^{1} S-{ }^{3} S$ & 9.3141 & $0-1$ & $7.293(4)$ & M1 & $6.5546(-6)$ \\
\hline & $1 s^{2}-1 s 2 p$ & ${ }^{1} S-{ }^{3} P$ & 9.2280 & $0-2$ & $1.060(7)$ & M2 & $2.3792(-1)$ \\
\hline \multirow[t]{2}{*}{13} & $1 s^{2}-1 s 2 s$ & ${ }^{1} S-{ }^{3} S$ & 7.8720 & $0-1$ & $1.672(5)$ & M1 & $9.0715(-6)$ \\
\hline & $1 s^{2}-1 s 2 p$ & ${ }^{1} S-{ }^{3} P$ & 7.8037 & $0-2$ & $2.080(7)$ & M2 & $2.0188(-1)$ \\
\hline \multirow[t]{2}{*}{14} & $1 s^{2}-1 s 2 s$ & ${ }^{1} S-{ }^{3} S$ & 6.7402 & $0-1$ & $3.598(5)$ & M1 & $1.2253(-5)$ \\
\hline & $1 s^{2}-1 s 2 p$ & ${ }^{1} S-{ }^{3} P$ & 6.6849 & $0-2$ & $3.873(7)$ & M2 & $1.7338(-1)$ \\
\hline \multirow[t]{2}{*}{15} & $1 s^{2}-1 s 2 s$ & ${ }^{1} S-{ }^{3} S$ & 5.8357 & $0-1$ & $7.333(5)$ & M1 & $1.6208(-5)$ \\
\hline & $1 s^{2}-1 s 2 p$ & ${ }^{1} S-{ }^{3} P$ & 5.7900 & $0-2$ & $6.895(7)$ & M2 & $1.5045(-1)$ \\
\hline \multirow[t]{2}{*}{16} & $1 s^{2}-1 s 2 s$ & ${ }^{1} S-{ }^{3} S$ & 5.1014 & $0-1$ & $1.426(6)$ & M1 & $2.1054(-5)$ \\
\hline & $1 s^{2}-1 s 2 p$ & ${ }^{1} S-{ }^{3} P$ & 5.0631 & $0-2$ & $1.181(8)$ & M2 & $1.3174(-1)$ \\
\hline \multirow[t]{2}{*}{17} & $1 s^{2}-1 s 2 s$ & ${ }^{1} S-{ }^{3} S$ & 4.4972 & $0-1$ & $2.661(6)$ & M1 & $2.6915(-5)$ \\
\hline & $1 s^{2}-1 s 2 p$ & ${ }^{1} S-{ }^{3} P$ & 4.4645 & $0-2$ & $1.955(8)$ & M2 & $1.1626(-1)$ \\
\hline \multirow[t]{2}{*}{18} & $1 s^{2}-1 s 2 s$ & ${ }^{1} S-{ }^{3} S$ & 3.9941 & $0-1$ & $4.787(6)$ & M1 & $3.3926(-5)$ \\
\hline & $1 s^{2}-1 s 2 p$ & ${ }^{1} S-{ }^{3} P$ & 3.9658 & $0-2$ & $3.141(8)$ & M2 & $1.0332(-1)$ \\
\hline 19 & $1 s^{2}-1 s 2 s$ & ${ }^{1} S-{ }^{3} S$ & 3.5707 & $0-1$ & $8.341(6)$ & M1 & $4.2231(-5)$ \\
\hline
\end{tabular}


Table 16: M1 and M2 transitions

\begin{tabular}{|c|c|c|c|c|c|c|c|}
\hline \multicolumn{3}{|c|}{ Transition } & \multicolumn{5}{|c|}{$A_{k i}$} \\
\hline$Z$ & Array & Multiplet & $\lambda(\AA)$ & $J_{i}-J_{k}$ & $\left(s^{-1}\right)$ & Type & S (a.u.) \\
\hline \multirow{3}{*}{20} & $1 s^{2}-1 s 2 p$ & ${ }^{1} S-{ }^{3} P$ & 3.5459 & $0-2$ & $4.914(8)$ & M2 & $9.2385(-2)$ \\
\hline & $1 s^{2}-1 s 2 s$ & ${ }^{1} S-{ }^{3} S$ & 3.2110 & $0-1$ & $1.412(7)$ & M1 & $5.1981(-5)$ \\
\hline & $1 s^{2}-1 s 2 p$ & ${ }^{1} S-{ }^{3} P$ & 3.1891 & $0-2$ & $7.510(8)$ & M2 & $8.3067(-2)$ \\
\hline \multirow[t]{2}{*}{21} & $1 s^{2}-1 s 2 s$ & ${ }^{1} S-{ }^{3} S$ & 2.9028 & $0-1$ & $2.328(7)$ & M1 & $6.3336(-5)$ \\
\hline & $1 s^{2}-1 s 2 p$ & ${ }^{1} S-{ }^{3} P$ & 2.8832 & $0-2$ & $1.123(9)$ & M2 & $7.5060(-2)$ \\
\hline \multirow[t]{2}{*}{22} & $1 s^{2}-1 s 2 s$ & ${ }^{1} S-{ }^{3} S$ & 2.6368 & $0-1$ & $3.750(7)$ & M1 & $7.6467(-5)$ \\
\hline & $1 s^{2}-1 s 2 p$ & ${ }^{1} S-{ }^{3} P$ & 2.6191 & $0-2$ & $1.648(9)$ & M2 & $6.8130(-2)$ \\
\hline \multirow[t]{2}{*}{23} & $1 s^{2}-1 s 2 s$ & ${ }^{1} S-{ }^{3} S$ & 2.4056 & $0-1$ & $5.913(7)$ & M1 & $9.1552(-5)$ \\
\hline & $1 s^{2}-1 s 2 p$ & ${ }^{1} S-{ }^{3} P$ & 2.3895 & $0-2$ & $2.377(9)$ & M2 & $6.2092(-2)$ \\
\hline \multirow[t]{2}{*}{24} & $1 s^{2}-1 s 2 s$ & ${ }^{1} S-{ }^{3} S$ & 2.2034 & $0-1$ & $9.143(7)$ & M1 & $1.0878(-4)$ \\
\hline & $1 s^{2}-1 s 2 p$ & ${ }^{1} S-{ }^{3} P$ & 2.1886 & $0-2$ & $3.373(9)$ & M2 & $5.6800(-2)$ \\
\hline \multirow[t]{2}{*}{25} & $1 s^{2}-1 s 2 s$ & ${ }^{1} S-{ }^{3} S$ & 2.0255 & $0-1$ & $1.389(8)$ & M1 & $1.2835(-4)$ \\
\hline & $1 s^{2}-1 s 2 p$ & ${ }^{1} S-{ }^{3} P$ & 2.0118 & $0-2$ & $4.718(9)$ & M2 & $5.2135(-2)$ \\
\hline \multirow[t]{2}{*}{26} & $1 s^{2}-1 s 2 s$ & ${ }^{1} S-{ }^{3} S$ & 1.8682 & $0-1$ & $2.075(8)$ & M1 & $1.5046(-4)$ \\
\hline & $1 s^{2}-1 s 2 p$ & ${ }^{1} S-{ }^{3} P$ & 1.8554 & $0-2$ & $6.510(9)$ & M2 & $4.8002(-2)$ \\
\hline \multirow[t]{2}{*}{27} & $1 s^{2}-1 s 2 s$ & ${ }^{1} S-{ }^{3} S$ & 1.7284 & $0-1$ & $3.053(8)$ & M1 & $1.7534(-4)$ \\
\hline & $1 s^{2}-1 s 2 p$ & ${ }^{1} S-{ }^{3} P$ & 1.7164 & $0-2$ & $8.873(9)$ & M2 & $4.4324(-2)$ \\
\hline \multirow[t]{2}{*}{28} & $1 s^{2}-1 s 2 s$ & ${ }^{1} S-{ }^{3} S$ & 1.6036 & $0-1$ & $4.431(8)$ & M1 & $2.0321(-4)$ \\
\hline & $1 s^{2}-1 s 2 p$ & ${ }^{1} S-{ }^{3} P$ & 1.5923 & $0-2$ & $1.195(10)$ & M2 & $4.1035(-2)$ \\
\hline \multirow[t]{2}{*}{29} & $1 s^{2}-1 s 2 s$ & ${ }^{1} S-{ }^{3} S$ & 1.4917 & $0-1$ & $6.346(8)$ & M1 & $2.3431(-4)$ \\
\hline & $1 s^{2}-1 s 2 p$ & ${ }^{1} S-{ }^{3} P$ & 1.4811 & $0-2$ & $1.594(10)$ & M2 & $3.8084(-2)$ \\
\hline \multirow[t]{2}{*}{30} & $1 s^{2}-1 s 2 s$ & ${ }^{1} S-{ }^{3} S$ & 1.3911 & $0-1$ & $8.981(8)$ & M1 & $2.6889(-4)$ \\
\hline & $1 s^{2}-1 s 2 p$ & ${ }^{1} S-{ }^{3} P$ & 1.3809 & $0-2$ & $2.104(10)$ & M2 & $3.5425(-2)$ \\
\hline \multirow[t]{2}{*}{31} & $1 s^{2}-1 s 2 s$ & ${ }^{1} S-{ }^{3} S$ & 1.3002 & $0-1$ & $1.257(9)$ & M1 & $3.0719(-4)$ \\
\hline & $1 s^{2}-1 s 2 p$ & ${ }^{1} S-{ }^{3} P$ & 1.2905 & $0-2$ & $2.751(10)$ & M2 & $3.3020(-2)$ \\
\hline \multirow[t]{2}{*}{32} & $1 s^{2}-1 s 2 s$ & ${ }^{1} S-{ }^{3} S$ & 1.2179 & $0-1$ & $1.740(9)$ & M1 & $3.4949(-4)$ \\
\hline & $1 s^{2}-1 s 2 p$ & ${ }^{1} S-{ }^{3} P$ & 1.2086 & $0-2$ & $3.567(10)$ & M2 & $3.0840(-2)$ \\
\hline \multirow[t]{2}{*}{33} & $1 s^{2}-1 s 2 s$ & ${ }^{1} S-{ }^{3} S$ & 1.1430 & $0-1$ & $2.385(9)$ & M1 & $3.9608(-4)$ \\
\hline & $1 s^{2}-1 s 2 p$ & ${ }^{1} S-{ }^{3} P$ & 1.1341 & $0-2$ & $4.587(10)$ & M2 & $2.8856(-2)$ \\
\hline \multirow[t]{2}{*}{34} & $1 s^{2}-1 s 2 s$ & ${ }^{1} S-{ }^{3} S$ & 1.0748 & $0-1$ & $3.239(9)$ & M1 & $4.4721(-4)$ \\
\hline & $1 s^{2}-1 s 2 p$ & ${ }^{1} S-{ }^{3} P$ & 1.0661 & $0-2$ & $5.855(10)$ & M2 & $2.7046(-2)$ \\
\hline \multirow[t]{2}{*}{35} & $1 s^{2}-1 s 2 s$ & ${ }^{1} S-{ }^{3} S$ & 1.0124 & $0-1$ & $4.360(9)$ & M1 & $5.0323(-4)$ \\
\hline & $1 s^{2}-1 s 2 p$ & ${ }^{1} S-{ }^{3} P$ & 1.0040 & $0-2$ & $7.420(10)$ & M2 & $2.5390(-2)$ \\
\hline \multirow[t]{2}{*}{36} & $1 s^{2}-1 s 2 s$ & ${ }^{1} S-{ }^{3} S$ & .95525 & $0-1$ & $5.822(9)$ & M1 & $5.6442(-4)$ \\
\hline & $1 s^{2}-1 s 2 p$ & ${ }^{1} S-{ }^{3} P$ & .94710 & $0-2$ & $9.341(10)$ & M2 & $2.3871(-2)$ \\
\hline \multirow[t]{2}{*}{37} & $1 s^{2}-1 s 2 s$ & ${ }^{1} S-{ }^{3} S$ & .90272 & $0-1$ & $7.714(9)$ & M1 & $6.3110(-4)$ \\
\hline & \multicolumn{7}{|c|}{ Continued on next page } \\
\hline
\end{tabular}


Table 16: M1 and M2 transitions

\begin{tabular}{|c|c|c|c|c|c|c|c|}
\hline \multicolumn{3}{|c|}{ Transition } & \multicolumn{5}{|c|}{$A_{k i}$} \\
\hline$Z$ & Array & Multiplet & $\lambda(\AA)$ & $J_{i}-J_{k}$ & $\left(s^{-1}\right)$ & Туре & S (a.u.) \\
\hline & $1 s^{2}-1 s 2 p$ & ${ }^{1} S-{ }^{3} P$ & .89479 & $0-2$ & $1.168(11)$ & M2 & $2.2475(-2)$ \\
\hline \multirow[t]{2}{*}{38} & $1 s^{2}-1 s 2 s$ & ${ }^{1} S-{ }^{3} S$ & .85434 & $0-1$ & $1.015(10)$ & M1 & $7.0363(-4)$ \\
\hline & $1 s^{2}-1 s 2 p$ & ${ }^{1} S-{ }^{3} P$ & .84661 & $0-2$ & $1.453(11)$ & M2 & $2.1188(-2)$ \\
\hline \multirow[t]{2}{*}{39} & $1 s^{2}-1 s 2 s$ & ${ }^{1} S-{ }^{3} S$ & .80969 & $0-1$ & $1.325(10)$ & M1 & $7.8231(-4)$ \\
\hline & $1 s^{2}-1 s 2 p$ & ${ }^{1} S-{ }^{3} P$ & .80214 & $0-2$ & $1.796(11)$ & M2 & $1.9999(-2)$ \\
\hline \multirow[t]{2}{*}{40} & $1 s^{2}-1 s 2 s$ & ${ }^{1} S-{ }^{3} S$ & .76840 & $0-1$ & $1.719(10)$ & M1 & $8.6756(-4)$ \\
\hline & $1 s^{2}-1 s 2 p$ & ${ }^{1} S-{ }^{3} P$ & .76099 & $0-2$ & $2.208(11)$ & M2 & $1.8900(-2)$ \\
\hline \multirow[t]{2}{*}{41} & $1 s^{2}-1 s 2 s$ & ${ }^{1} S-{ }^{3} S$ & .73012 & $0-1$ & $2.217(10)$ & M1 & $9.5972(-4)$ \\
\hline & $1 s^{2}-1 s 2 p$ & ${ }^{1} S-{ }^{3} P$ & .72286 & $0-2$ & $2.701(11)$ & M2 & $1.7880(-2)$ \\
\hline \multirow[t]{2}{*}{42} & $1 s^{2}-1 s 2 s$ & ${ }^{1} S-{ }^{3} S$ & .69459 & $0-1$ & $2.842(10)$ & M1 & $1.0592(-3)$ \\
\hline & $1 s^{2}-1 s 2 p$ & ${ }^{1} S-{ }^{3} P$ & .68745 & $0-2$ & $3.289(11)$ & M2 & $1.6933(-2)$ \\
\hline \multirow[t]{2}{*}{43} & $1 s^{2}-1 s 2 s$ & ${ }^{1} S-{ }^{3} S$ & .66153 & $0-1$ & $3.622(10)$ & M1 & $1.1663(-3)$ \\
\hline & $1 s^{2}-1 s 2 p$ & ${ }^{1} S-{ }^{3} P$ & .65451 & $0-2$ & $3.985(11)$ & M2 & $1.6052(-2)$ \\
\hline \multirow[t]{2}{*}{44} & $1 s^{2}-1 s 2 s$ & ${ }^{1} S-{ }^{3} S$ & .63073 & $0-1$ & $4.592(10)$ & M1 & $1.2816(-3)$ \\
\hline & $1 s^{2}-1 s 2 p$ & ${ }^{1} S-{ }^{3} P$ & .62382 & $0-2$ & $4.808(11)$ & M2 & $1.5230(-2)$ \\
\hline \multirow[t]{2}{*}{45} & $1 s^{2}-1 s 2 s$ & ${ }^{1} S-{ }^{3} S$ & .60198 & $0-1$ & $5.792(10)$ & M1 & $1.4054(-3)$ \\
\hline & $1 s^{2}-1 s 2 p$ & ${ }^{1} S-{ }^{3} P$ & .59517 & $0-2$ & $5.775(11)$ & M2 & $1.4464(-2)$ \\
\hline \multirow[t]{2}{*}{46} & $1 s^{2}-1 s 2 s$ & ${ }^{1} S-{ }^{3} S$ & .57511 & $0-1$ & $7.270(10)$ & M1 & $1.5381(-3)$ \\
\hline & $1 s^{2}-1 s 2 p$ & ${ }^{1} S-{ }^{3} P$ & .56838 & $0-2$ & $6.910(11)$ & M2 & $1.3747(-2)$ \\
\hline \multirow[t]{2}{*}{47} & $1 s^{2}-1 s 2 s$ & ${ }^{1} S-{ }^{3} S$ & .54996 & $0-1$ & $9.083(10)$ & M1 & $1.6803(-3)$ \\
\hline & $1 s^{2}-1 s 2 p$ & ${ }^{1} S-{ }^{3} P$ & .54331 & $0-2$ & $8.237(11)$ & M2 & $1.3076(-2)$ \\
\hline \multirow[t]{2}{*}{48} & $1 s^{2}-1 s 2 s$ & ${ }^{1} S-{ }^{3} S$ & .52637 & $0-1$ & $1.130(11)$ & M1 & $1.8324(-3)$ \\
\hline & $1 s^{2}-1 s 2 p$ & ${ }^{1} S-{ }^{3} P$ & .51979 & $0-2$ & $9.781(11)$ & M2 & $1.2447(-2)$ \\
\hline \multirow[t]{2}{*}{49} & $1 s^{2}-1 s 2 s$ & ${ }^{1} S-{ }^{3} S$ & .50423 & $0-1$ & $1.399(11)$ & M1 & $1.9948(-3)$ \\
\hline & $1 s^{2}-1 s 2 p$ & ${ }^{1} S-{ }^{3} P$ & .49772 & $0-2$ & $1.157(12)$ & M2 & $1.1856(-2)$ \\
\hline \multirow[t]{2}{*}{50} & $1 s^{2}-1 s 2 s$ & ${ }^{1} S-{ }^{3} S$ & .48342 & $0-1$ & $1.726(11)$ & M1 & $2.1681(-3)$ \\
\hline & $1 s^{2}-1 s 2 p$ & ${ }^{1} S-{ }^{3} P$ & .47697 & $0-2$ & $1.365(12)$ & M2 & $1.1301(-2)$ \\
\hline \multirow[t]{2}{*}{51} & $1 s^{2}-1 s 2 s$ & ${ }^{1} S-{ }^{3} S$ & .46384 & $0-1$ & $2.120(11)$ & M1 & $2.3529(-3)$ \\
\hline & $1 s^{2}-1 s 2 p$ & ${ }^{1} S-{ }^{3} P$ & .45744 & $0-2$ & $1.605(12)$ & M2 & $1.0778(-2)$ \\
\hline \multirow[t]{2}{*}{52} & $1 s^{2}-1 s 2 s$ & ${ }^{1} S-{ }^{3} S$ & .44538 & $0-1$ & $2.595(11)$ & M1 & $2.5495(-3)$ \\
\hline & $1 s^{2}-1 s 2 p$ & ${ }^{1} S-{ }^{3} P$ & .43903 & $0-2$ & $1.881(12)$ & M2 & $1.0286(-2)$ \\
\hline \multirow[t]{2}{*}{53} & $1 s^{2}-1 s 2 s$ & ${ }^{1} S-{ }^{3} S$ & .42796 & $0-1$ & $3.164(11)$ & M1 & $2.7586(-3)$ \\
\hline & $1 s^{2}-1 s 2 p$ & ${ }^{1} S-{ }^{3} P$ & .42166 & $0-2$ & $2.197(12)$ & M2 & $9.8219(-3)$ \\
\hline \multirow[t]{2}{*}{54} & $1 s^{2}-1 s 2 s$ & ${ }^{1} S-{ }^{3} S$ & .41151 & $0-1$ & $3.846(11)$ & M1 & $2.9807(-3)$ \\
\hline & $1 s^{2}-1 s 2 p$ & ${ }^{1} S-{ }^{3} P$ & .40526 & $0-2$ & $2.560(12)$ & M2 & $9.3835(-3)$ \\
\hline \multirow[t]{2}{*}{55} & $1 s^{2}-1 s 2 s$ & ${ }^{1} S-{ }^{3} S$ & .39596 & $0-1$ & $4.658(11)$ & M1 & $3.2165(-3)$ \\
\hline & \multicolumn{7}{|c|}{ Continued on next page } \\
\hline
\end{tabular}


Table 16: M1 and M2 transitions

\begin{tabular}{|c|c|c|c|c|c|c|c|}
\hline \multicolumn{3}{|c|}{ Transition } & \multicolumn{5}{|c|}{$A_{k i}$} \\
\hline \multirow[t]{2}{*}{$Z$} & Array & Multiplet & $\lambda(\AA)$ & $J_{i}-J_{k}$ & $\left(s^{-1}\right)$ & Туре & S (a.u.) \\
\hline & $1 s^{2}-1 s 2 p$ & ${ }^{1} S-{ }^{3} P$ & .38974 & $0-2$ & $2.974(12)$ & M2 & $8.9691(-3)$ \\
\hline \multirow[t]{2}{*}{56} & $1 s^{2}-1 s 2 s$ & ${ }^{1} S-{ }^{3} S$ & .38124 & $0-1$ & $5.625(11)$ & M1 & $3.4665(-3)$ \\
\hline & $1 s^{2}-1 s 2 p$ & ${ }^{1} S-{ }^{3} P$ & .37506 & $0-2$ & $3.446(12)$ & M2 & $8.5770(-3)$ \\
\hline \multirow[t]{2}{*}{57} & $1 s^{2}-1 s 2 s$ & ${ }^{1} S-{ }^{3} S$ & .36729 & $0-1$ & $6.771(11)$ & M1 & $3.7313(-3)$ \\
\hline & $1 s^{2}-1 s 2 p$ & ${ }^{1} S-{ }^{3} P$ & .36115 & $0-2$ & $3.983(12)$ & M2 & $8.2057(-3)$ \\
\hline \multirow[t]{2}{*}{58} & $1 s^{2}-1 s 2 s$ & ${ }^{1} S-{ }^{3} S$ & .35406 & $0-1$ & $8.126(11)$ & M1 & $4.0117(-3)$ \\
\hline & $1 s^{2}-1 s 2 p$ & ${ }^{1} S-{ }^{3} P$ & .34795 & $0-2$ & $4.592(12)$ & M2 & $7.8537(-3)$ \\
\hline \multirow[t]{2}{*}{59} & $1 s^{2}-1 s 2 s$ & ${ }^{1} S-{ }^{3} S$ & .34151 & $0-1$ & $9.725(11)$ & M1 & $4.3082(-3)$ \\
\hline & $1 s^{2}-1 s 2 p$ & ${ }^{1} S-{ }^{3} P$ & .33542 & $0-2$ & $5.281(12)$ & M2 & $7.5198(-3)$ \\
\hline \multirow[t]{2}{*}{60} & $1 s^{2}-1 s 2 s$ & ${ }^{1} S-{ }^{3} S$ & .32958 & $0-1$ & $1.161(12)$ & M1 & $4.6215(-3)$ \\
\hline & $1 s^{2}-1 s 2 p$ & ${ }^{1} S-{ }^{3} P$ & .32352 & $0-2$ & $6.060(12)$ & M2 & $7.2027(-3)$ \\
\hline \multirow[t]{2}{*}{61} & $1 s^{2}-1 s 2 s$ & ${ }^{1} S-{ }^{3} S$ & .31824 & $0-1$ & $1.382(12)$ & M1 & $4.9523(-3)$ \\
\hline & $1 s^{2}-1 s 2 p$ & ${ }^{1} S-{ }^{3} P$ & .31220 & $0-2$ & $6.939(12)$ & M2 & $6.9015(-3)$ \\
\hline \multirow[t]{2}{*}{62} & $1 s^{2}-1 s 2 s$ & ${ }^{1} S-{ }^{3} S$ & .30744 & $0-1$ & $1.640(12)$ & M1 & $5.3014(-3)$ \\
\hline & $1 s^{2}-1 s 2 p$ & ${ }^{1} S-{ }^{3} P$ & .30143 & $0-2$ & 7.927(12) & M2 & $6.6150(-3)$ \\
\hline \multirow[t]{2}{*}{63} & $1 s^{2}-1 s 2 s$ & ${ }^{1} S-{ }^{3} S$ & .29715 & $0-1$ & $1.943(12)$ & M1 & $5.6695(-3)$ \\
\hline & $1 s^{2}-1 s 2 p$ & ${ }^{1} S-{ }^{3} P$ & .29116 & $0-2$ & $9.038(12)$ & M2 & $6.3422(-3)$ \\
\hline \multirow[t]{2}{*}{64} & $1 s^{2}-1 s 2 s$ & ${ }^{1} S-{ }^{3} S$ & .28735 & $0-1$ & $2.295(12)$ & M1 & $6.0574(-3)$ \\
\hline & $1 s^{2}-1 s 2 p$ & ${ }^{1} S-{ }^{3} P$ & .28138 & $0-2$ & $1.028(13)$ & M2 & $6.0825(-3)$ \\
\hline \multirow[t]{2}{*}{65} & $1 s^{2}-1 s 2 s$ & ${ }^{1} S-{ }^{3} S$ & .27799 & $0-1$ & $2.706(12)$ & M1 & $6.4659(-3)$ \\
\hline & $1 s^{2}-1 s 2 p$ & ${ }^{1} S-{ }^{3} P$ & .27204 & $0-2$ & $1.168(13)$ & M2 & $5.8349(-3)$ \\
\hline \multirow[t]{2}{*}{66} & $1 s^{2}-1 s 2 s$ & ${ }^{1} S-{ }^{3} S$ & .26906 & $0-1$ & $3.183(12)$ & M1 & $6.8959(-3)$ \\
\hline & $1 s^{2}-1 s 2 p$ & ${ }^{1} S-{ }^{3} P$ & .26312 & $0-2$ & $1.324(13)$ & M2 & $5.5988(-3)$ \\
\hline \multirow[t]{2}{*}{67} & $1 s^{2}-1 s 2 s$ & ${ }^{1} S-{ }^{3} S$ & .26053 & $0-1$ & $3.736(12)$ & M1 & $7.3478(-3)$ \\
\hline & $1 s^{2}-1 s 2 p$ & ${ }^{1} S-{ }^{3} P$ & .25461 & $0-2$ & $1.498(13)$ & M2 & $5.3735(-3)$ \\
\hline \multirow[t]{2}{*}{68} & $1 s^{2}-1 s 2 s$ & ${ }^{1} S-{ }^{3} S$ & .25237 & $0-1$ & $4.376(12)$ & M1 & $7.8233(-3)$ \\
\hline & $1 s^{2}-1 s 2 p$ & ${ }^{1} S-{ }^{3} P$ & .24646 & $0-2$ & $1.691(13)$ & M2 & $5.1582(-3)$ \\
\hline \multirow[t]{2}{*}{69} & $1 s^{2}-1 s 2 s$ & ${ }^{1} S-{ }^{3} S$ & .24457 & $0-1$ & $5.115(12)$ & M1 & $8.3225(-3)$ \\
\hline & $1 s^{2}-1 s 2 p$ & ${ }^{1} S-{ }^{3} P$ & .23867 & $0-2$ & $1.907(13)$ & M2 & $4.9526(-3)$ \\
\hline \multirow[t]{2}{*}{70} & $1 s^{2}-1 s 2 s$ & ${ }^{1} S-{ }^{3} S$ & .23709 & $0-1$ & $5.968(12)$ & M1 & $8.8466(-3)$ \\
\hline & $1 s^{2}-1 s 2 p$ & ${ }^{1} S-{ }^{3} P$ & .23121 & $0-2$ & $2.146(13)$ & M2 & $4.7560(-3)$ \\
\hline \multirow[t]{2}{*}{71} & $1 s^{2}-1 s 2 s$ & ${ }^{1} S-{ }^{3} S$ & .22993 & $0-1$ & $6.950(12)$ & M1 & $9.3969(-3)$ \\
\hline & $1 s^{2}-1 s 2 p$ & ${ }^{1} S-{ }^{3} P$ & .22406 & $0-2$ & $2.412(13)$ & M2 & $4.5679(-3)$ \\
\hline \multirow[t]{2}{*}{72} & $1 s^{2}-1 s 2 s$ & ${ }^{1} S-{ }^{3} S$ & .22307 & $0-1$ & $8.079(12)$ & M1 & $9.9738(-3)$ \\
\hline & $1 s^{2}-1 s 2 p$ & ${ }^{1} S-{ }^{3} P$ & .21721 & $0-2$ & $2.706(13)$ & M2 & $4.3879(-3)$ \\
\hline \multirow[t]{2}{*}{73} & $1 s^{2}-1 s 2 s$ & ${ }^{1} S-{ }^{3} S$ & .21649 & $0-1$ & $9.375(12)$ & M1 & $1.0579(-2)$ \\
\hline & \multicolumn{7}{|c|}{ Continued on next page } \\
\hline
\end{tabular}


Table 16: M1 and M2 transitions

\begin{tabular}{|c|c|c|c|c|c|c|c|}
\hline \multicolumn{3}{|c|}{ Transition } & \multicolumn{5}{|c|}{$A_{k i}$} \\
\hline \multirow[t]{2}{*}{$Z$} & Array & Multiplet & $\lambda(\AA)$ & $J_{i}-J_{k}$ & $\left(s^{-1}\right)$ & Туре & S (a.u.) \\
\hline & $1 s^{2}-1 s 2 p$ & ${ }^{1} S-{ }^{3} P$ & .21063 & $0-2$ & $3.032(13)$ & M2 & $4.2154(-3)$ \\
\hline \multirow[t]{2}{*}{74} & $1 s^{2}-1 s 2 s$ & ${ }^{1} S-{ }^{3} S$ & .21017 & $0-1$ & $1.086(13)$ & M1 & $1.1213(-2)$ \\
\hline & $1 s^{2}-1 s 2 p$ & ${ }^{1} S-{ }^{3} P$ & .20432 & $0-2$ & $3.392(13)$ & M2 & $4.0503(-3)$ \\
\hline \multirow[t]{2}{*}{75} & $1 s^{2}-1 s 2 s$ & ${ }^{1} S-{ }^{3} S$ & .20410 & $0-1$ & $1.256(13)$ & M1 & $1.1877(-2)$ \\
\hline & $1 s^{2}-1 s 2 p$ & ${ }^{1} S-{ }^{3} P$ & .19826 & $0-2$ & $3.789(13)$ & M2 & $3.8919(-3)$ \\
\hline \multirow[t]{2}{*}{76} & $1 s^{2}-1 s 2 s$ & ${ }^{1} S-{ }^{3} S$ & .19827 & $0-1$ & $1.450(13)$ & M1 & $1.2572(-2)$ \\
\hline & $1 s^{2}-1 s 2 p$ & ${ }^{1} S-{ }^{3} P$ & .19243 & $0-2$ & $4.226(13)$ & M2 & $3.7400(-3)$ \\
\hline \multirow[t]{2}{*}{77} & $1 s^{2}-1 s 2 s$ & ${ }^{1} S-{ }^{3} S$ & .19266 & $0-1$ & $1.672(13)$ & M1 & $1.3300(-2)$ \\
\hline & $1 s^{2}-1 s 2 p$ & ${ }^{1} S-{ }^{3} P$ & .18683 & $0-2$ & $4.708(13)$ & M2 & $3.5942(-3)$ \\
\hline \multirow[t]{2}{*}{78} & $1 s^{2}-1 s 2 s$ & ${ }^{1} S-{ }^{3} S$ & .18727 & $0-1$ & $1.925(13)$ & M1 & $1.4061(-2)$ \\
\hline & $1 s^{2}-1 s 2 p$ & ${ }^{1} S-{ }^{3} P$ & .18145 & $0-2$ & $5.237(13)$ & M2 & $3.4543(-3)$ \\
\hline \multirow[t]{2}{*}{79} & $1 s^{2}-1 s 2 s$ & ${ }^{1} S-{ }^{3} S$ & .18208 & $0-1$ & $2.213(13)$ & M1 & $1.4857(-2)$ \\
\hline & $1 s^{2}-1 s 2 p$ & ${ }^{1} S-{ }^{3} P$ & .17626 & $0-2$ & $5.819(13)$ & M2 & $3.3200(-3)$ \\
\hline \multirow[t]{2}{*}{80} & $1 s^{2}-1 s 2 s$ & ${ }^{1} S-{ }^{3} S$ & .17708 & $0-1$ & $2.540(13)$ & M1 & $1.5689(-2)$ \\
\hline & $1 s^{2}-1 s 2 p$ & ${ }^{1} S-{ }^{3} P$ & .17127 & $0-2$ & $6.457(13)$ & M2 & $3.1909(-3)$ \\
\hline \multirow[t]{2}{*}{81} & $1 s^{2}-1 s 2 s$ & ${ }^{1} S-{ }^{3} S$ & .17226 & $0-1$ & 2.913(13) & M1 & $1.6558(-2)$ \\
\hline & $1 s^{2}-1 s 2 p$ & ${ }^{1} S-{ }^{3} P$ & .16645 & $0-2$ & $7.157(13)$ & M2 & $3.0668(-3)$ \\
\hline \multirow[t]{2}{*}{82} & $1 s^{2}-1 s 2 s$ & ${ }^{1} S-{ }^{3} S$ & .16762 & $0-1$ & $3.335(13)$ & M1 & $1.7466(-2)$ \\
\hline & $1 s^{2}-1 s 2 p$ & ${ }^{1} S-{ }^{3} P$ & .16181 & $0-2$ & $7.923(13)$ & M2 & $2.9476(-3)$ \\
\hline \multirow[t]{2}{*}{83} & $1 s^{2}-1 s 2 s$ & ${ }^{1} S-{ }^{3} S$ & .16314 & $0-1$ & $3.813(13)$ & M1 & $1.8414(-2)$ \\
\hline & $1 s^{2}-1 s 2 p$ & ${ }^{1} S-{ }^{3} P$ & .15734 & $0-2$ & $8.761(13)$ & M2 & $2.8329(-3)$ \\
\hline \multirow[t]{2}{*}{84} & $1 s^{2}-1 s 2 s$ & ${ }^{1} S-{ }^{3} S$ & .15882 & $0-1$ & $4.355(13)$ & M1 & $1.9404(-2)$ \\
\hline & $1 s^{2}-1 s 2 p$ & ${ }^{1} S-{ }^{3} P$ & .15302 & $0-2$ & $9.676(13)$ & M2 & $2.7224(-3)$ \\
\hline \multirow[t]{2}{*}{85} & $1 s^{2}-1 s 2 s$ & ${ }^{1} S-{ }^{3} S$ & .15465 & $0-1$ & $4.968(13)$ & M1 & $2.0437(-2)$ \\
\hline & $1 s^{2}-1 s 2 p$ & ${ }^{1} S-{ }^{3} P$ & .14885 & $0-2$ & $1.068(14)$ & M2 & $2.6163(-3)$ \\
\hline \multirow[t]{2}{*}{86} & $1 s^{2}-1 s 2 s$ & ${ }^{1} S-{ }^{3} S$ & .15063 & $0-1$ & $5.660(13)$ & M1 & $2.1513(-2)$ \\
\hline & $1 s^{2}-1 s 2 p$ & ${ }^{1} S-{ }^{3} P$ & .14483 & $0-2$ & $1.176(14)$ & M2 & $2.5141(-3)$ \\
\hline \multirow[t]{2}{*}{87} & $1 s^{2}-1 s 2 s$ & ${ }^{1} S-{ }^{3} S$ & .14673 & $0-1$ & $6.442(13)$ & M1 & $2.2637(-2)$ \\
\hline & $1 s^{2}-1 s 2 p$ & ${ }^{1} S-{ }^{3} P$ & .14094 & $0-2$ & $1.295(14)$ & M2 & $2.4155(-3)$ \\
\hline \multirow[t]{2}{*}{88} & $1 s^{2}-1 s 2 s$ & ${ }^{1} S-{ }^{3} S$ & .14297 & $0-1$ & $7.325(13)$ & M1 & $2.3808(-2)$ \\
\hline & $1 s^{2}-1 s 2 p$ & ${ }^{1} S-{ }^{3} P$ & .13718 & $0-2$ & $1.425(14)$ & M2 & $2.3207(-3)$ \\
\hline \multirow[t]{2}{*}{89} & $1 s^{2}-1 s 2 s$ & ${ }^{1} S-{ }^{3} S$ & .13933 & $0-1$ & $8.319(13)$ & M1 & $2.5029(-2)$ \\
\hline & $1 s^{2}-1 s 2 p$ & ${ }^{1} S-{ }^{3} P$ & .13354 & $0-2$ & $1.565(14)$ & M2 & $2.2292(-3)$ \\
\hline \multirow[t]{2}{*}{90} & $1 s^{2}-1 s 2 s$ & ${ }^{1} S-{ }^{3} S$ & .13581 & $0-1$ & $9.439(13)$ & M1 & $2.6300(-2)$ \\
\hline & $1 s^{2}-1 s 2 p$ & ${ }^{1} S-{ }^{3} P$ & .13002 & $0-2$ & $1.718(14)$ & M2 & $2.1411(-3)$ \\
\hline \multirow[t]{2}{*}{91} & $1 s^{2}-1 s 2 s$ & ${ }^{1} S-{ }^{3} S$ & .13241 & $0-1$ & $1.070(14)$ & M1 & $2.7626(-2)$ \\
\hline & \multicolumn{7}{|c|}{ Continued on next page } \\
\hline
\end{tabular}


Table 16: M1 and M2 transitions

\begin{tabular}{cccccccc}
\hline$Z$ & $\begin{array}{c}\text { Transition } \\
\text { Array }\end{array}$ & Multiplet & $\lambda(\AA)$ & $J_{i}-J_{k}$ & $\begin{array}{c}A_{k i} \\
\left(s^{-1}\right)\end{array}$ & Type & S (a.u.) \\
\hline \multirow{3}{*}{92} & $1 s^{2}-1 s 2 p$ & ${ }^{1} S-{ }^{3} P$ & .12662 & $0-2$ & $1.884(14)$ & M2 & $2.0561(-3)$ \\
& $1 s^{2}-1 s 2 s$ & ${ }^{1} S-3 S$ & .12911 & $0-1$ & $1.212(14)$ & M1 & $2.9005(-2)$ \\
& $1 s^{2}-1 s 2 p$ & ${ }^{1} S-{ }^{3} P$ & .12332 & $0-2$ & $2.064(14)$ & M2 & $1.9743(-3)$ \\
93 & $1 s^{2}-1 s 2 s$ & ${ }^{1} S-3 S$ & .12592 & $0-1$ & $1.371(14)$ & M1 & $3.0442(-2)$ \\
& $1 s^{2}-1 s 2 p$ & ${ }^{1} S-3 P$ & .12012 & $0-2$ & $2.260(14)$ & M2 & $1.8952(-3)$ \\
94 & $1 s^{2}-1 s 2 s$ & ${ }^{1} S-3 S$ & .12282 & $0-1$ & $1.550(14)$ & M1 & $3.1935(-2)$ \\
& $1 s^{2}-1 s 2 p$ & ${ }^{1} S-{ }^{3} P$ & .11703 & $0-2$ & $2.471(14)$ & M2 & $1.8190(-3)$ \\
95 & $1 s^{2}-1 s 2 s$ & ${ }^{1} S-3 S$ & .11982 & $0-1$ & $1.751(14)$ & M1 & $3.3493(-2)$ \\
& $1 s^{2}-1 s 2 p$ & ${ }^{1} S-{ }^{3} P$ & .11402 & $0-2$ & $2.700(14)$ & M2 & $1.7454(-3)$ \\
96 & $1 s^{2}-1 s 2 s$ & ${ }^{1} S-3 S$ & .11691 & $0-1$ & $1.976(14)$ & M1 & $3.5110(-2)$ \\
& $1 s^{2}-1 s 2 p$ & ${ }^{1} S-{ }^{3} P$ & .11111 & $0-2$ & $2.948(14)$ & M2 & $1.6745(-3)$ \\
97 & $1 s^{2}-1 s 2 s$ & ${ }^{1} S-3 S$ & .11408 & $0-1$ & $2.228(14)$ & M1 & $3.6794(-2)$ \\
& $1 s^{2}-1 s 2 p$ & ${ }^{1} S-{ }^{3} P$ & .10829 & $0-2$ & $3.216(14)$ & M2 & $1.6059(-3)$ \\
98 & $1 s^{2}-1 s 2 s$ & ${ }^{1} S-3 S$ & .11135 & $0-1$ & $2.510(14)$ & M1 & $3.8543(-2)$ \\
& $1 s^{2}-1 s 2 p$ & ${ }^{1} S-{ }^{3} P$ & .10555 & $0-2$ & $3.506(14)$ & M2 & $1.5398(-3)$ \\
99 & $1 s^{2}-1 s 2 s$ & ${ }^{1} S-3 S$ & .10868 & $0-1$ & $2.827(14)$ & M1 & $4.0362(-2)$ \\
& $1 s^{2}-1 s 2 p$ & ${ }^{1} S-{ }^{3} P$ & .10288 & $0-2$ & $3.819(14)$ & M2 & $1.4760(-3)$ \\
100 & $1 s^{2}-1 s 2 s$ & ${ }^{1} S-3 S$ & .10610 & $0-1$ & $3.181(14)$ & M1 & $4.2252(-2)$ \\
& $1 s^{2}-1 s 2 p$ & ${ }^{1} S-{ }^{3} P$ & .10029 & $0-2$ & $4.156(14)$ & M2 & $1.4143(-3)$ \\
\hline \hline
\end{tabular}

